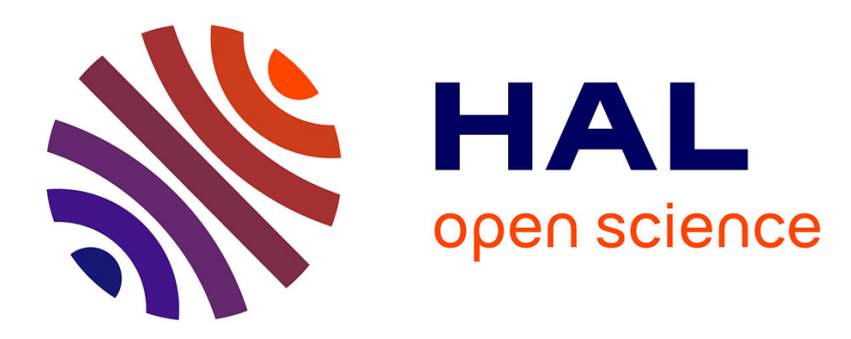

\title{
Semigroup estimates and stability/instability results for the linearized three waves interaction equations \\ David Chiron
}

\section{To cite this version:}

David Chiron. Semigroup estimates and stability/instability results for the linearized three waves interaction equations. Revista Matematica Complutense, 2012, 25, pp.285-333. 10.1007/s13163-0110070-y . hal-00809134

\section{HAL Id: hal-00809134 \\ https://hal.science/hal-00809134}

Submitted on 8 Apr 2013

HAL is a multi-disciplinary open access archive for the deposit and dissemination of scientific research documents, whether they are published or not. The documents may come from teaching and research institutions in France or abroad, or from public or private research centers.
L'archive ouverte pluridisciplinaire HAL, est destinée au dépôt et à la diffusion de documents scientifiques de niveau recherche, publiés ou non, émanant des établissements d'enseignement et de recherche français ou étrangers, des laboratoires publics ou privés. 


\title{
Semigroup estimates and stability/instability results for the linearized three waves interaction equations
}

\author{
David Chiron \\ Laboratoire J.A. Dieudonné, \\ Université de Nice-Sophia Antipolis, Parc Valrose, \\ 06108 Nice Cedex 02, France \\ E-mail: chiron@unice.fr
}

\begin{abstract}
We consider the three waves interaction system and its linearization (the "pump-wave approximation"). We give some estimates on the semigroup as well as stability or instability results for the linearized problem in suitable norms. We work in the whole space and with periodic boundary condition, and our analysis relies on energy estimates and not on the complete integrability of the system.
\end{abstract}

Key-words: three waves interaction equations, pump-wave approximation, stability, eigenvalue, semigroup. MSC (2010): 35Q99, 35F05, 35F20.

\section{The three waves interaction equations}

In this paper, we consider the three waves interaction system in dimension $d$

$$
\left\{\begin{array}{l}
\partial_{t} A_{1}+\mathrm{v}_{1} \cdot \nabla A_{1}+i \sigma_{1} \bar{A}_{2} \bar{A}_{3}=0 \\
\partial_{t} A_{2}+\mathrm{v}_{2} \cdot \nabla A_{2}+i \sigma_{2} \bar{A}_{3} \bar{A}_{1}=0 \\
\partial_{t} A_{3}+\mathrm{v}_{3} \cdot \nabla A_{3}+i \sigma_{3} \bar{A}_{1} \bar{A}_{2}=0 .
\end{array}\right.
$$

The amplitudes $A_{\ell}$ are complex-valued, the speeds $\mathrm{v}_{\ell}$ are fixed vectors in $\mathbb{R}^{d}$, and the coupling constants $\sigma_{\ell}$ are real-valued. To take into account a domain with periodic condition or in the whole space, the system (TWI) will be posed in

$$
\Omega=\Omega_{\Lambda} \equiv \prod_{j=1}^{d} \mathbb{R} /\left(2 \pi \Lambda_{j} \mathbb{Z}\right),
$$

with $\Lambda=\left(\Lambda_{j}\right)_{1 \leq j \leq d} \in(0,+\infty]^{d}$ and the convention that $\mathbb{R} /\left(2 \pi \Lambda_{j} \mathbb{Z}\right)=\mathbb{R}$ if $\Lambda_{j}=+\infty$.

The system (TWI) appears in various areas of physics: plasma physics, fluid mechanics, optics, acoustics, mechanical or electrical oscillations... We refer, for instance, to the book [6] and to the paper [8]. One classical way to derive the system (TWI) is as follows. Consider a scalar wave equation with quadratic nonlinearity

$$
\partial_{\mathrm{t}} u+\mathcal{Q} u+\mathcal{R}\left(u^{2}\right)=0
$$

(for example the $\mathrm{KdV}$ equation, the KP equation... but this can be extended to systems). The operators $\mathcal{Q}$ and $\mathcal{R}$ are pseudo-differential operators of symbol $i Q=i Q(k) \in i \mathbb{R}$ and $i R=i R(k) \in i \mathbb{R}$ respectively, and the dispersion relation for the linearized equation is $\omega=Q(k)$. If some wave numbers $\left(k_{1}, k_{2}, k_{3}\right)$ form a resonant triad, i.e.

$$
k_{1}+k_{2}+k_{3}=0, \quad \omega_{1}+\omega_{2}+\omega_{3}=0, \quad \text { with } \quad \omega_{\ell} \equiv Q\left(k_{\ell}\right), \quad \ell=1,2,3,
$$


we can search for a solution $u=u(\mathrm{t}, \mathrm{x})$ of the wave equation (1) which is the sum of three wave packets of small amplitude with these resonant wave numbers $\left(k_{1}, k_{2}, k_{3}\right)$ and slowly modulated. More precisely, $u$ is assumed to have an expansion of the form

$$
u(\mathrm{t}, \mathrm{x})=\varepsilon \sum_{\ell=1}^{3} A_{\ell}(t=\varepsilon \mathrm{t}, x=\varepsilon \mathrm{x}) \mathrm{e}^{i\left(k_{\ell} \cdot \mathrm{x}-\omega_{\ell} \mathrm{t}\right)}+\text { c.c. }+\mathcal{O}\left(\varepsilon^{2}\right) .
$$

Notice that the lengthscale of the modulation is of the same order as the typical packet amplitude. Collecting the terms of order $\varepsilon^{2}$ and cancelling out the secular terms, we infer that, on a formal level, $A_{1}, A_{2}, A_{3}$ satisfy (on the scales $(t=\varepsilon \mathrm{t}, x=\varepsilon \mathrm{x})$ ), the system (TWI), with $\mathrm{v}_{\ell}=\nabla Q\left(k_{\ell}\right)$ (the velocity group for the wave number $k_{\ell}$ ) and (real-valued) coefficients $\sigma_{\ell}=R\left(k_{\ell}\right)$ depending on the operator $\mathcal{R}$. In optics, for instance, the time coordinate can be a space coordinate.

For the system (TWI), the so-called Manley-Rowe relations give conserved quantities:

$$
\frac{d}{d t} \int_{\Omega} \sigma_{3}\left|A_{2}\right|^{2}-\sigma_{2}\left|A_{3}\right|^{2} d x=\frac{d}{d t} \int_{\Omega} \sigma_{1}\left|A_{3}\right|^{2}-\sigma_{3}\left|A_{1}\right|^{2} d x=\frac{d}{d t} \int_{\Omega} \sigma_{2}\left|A_{1}\right|^{2}-\sigma_{1}\left|A_{2}\right|^{2} d x=0 .
$$

Actually, a Lax pair can be found for the system (TWI) (see [13] for $d=1$, [12] for $d=2$ and [14] for $d=3$ ) thus is a completely integrable system for which inverse scattering can be used. Therefore, there exists an infinite sequence of (formally) conserved quantities (see [1], [7]). Notice however that they do not give $H^{s}$ bounds uniform in time (as for the KdV equation for instance). Furthermore, explicit soliton solutions can be computed, and blow-up can occur in finite time (under some conditions on the parameters, see [13]). One can also focus on solutions depending only on time or on space (we get a system of ODE's). In order to use inverse scattering, we have to work with data and solutions sufficiently smooth and strongly localized in space. In the physical literature, characteristic functions are of frequent use. The inverse scattering has been well developed when $\Omega$ is the whole space $\mathbb{R}^{d}$, but is always more difficult in the periodic setting. The paper [3] deals rigorously with the inverse scattering problem in the Schwartz space on the line. It is restricted to "generic data", but this fact has been removed in [15]. In general, it is not always clear in which functional spaces the data and the solution are, since the solution obtained by inverse scattering can lie in a space larger than the one used for the initial data. The paper [16] clarifies this point for data in Sobolev spaces with weight $W_{m}^{s, 2}$ defined below. In this paper, we shall work in $L^{p}$ and Sobolev spaces and shall not use inverse scattering techniques.

\subsection{A well-posedness result}

We denote $J \equiv\left\{1 \leq j \leq d, \Lambda_{j}=+\infty\right\}$ and, for $x \in \mathbb{R}^{d}, x^{J} \equiv\left(x_{j}\right)_{j \in J} \in \mathbb{R}^{J} \subset \mathbb{R}^{d}$. We define for $s$, $m \in \mathbb{N}$ and $1 \leq p \leq \infty$ the Sobolev space with weight

$$
W_{m}^{s, p}(\Omega) \equiv\left\{f \in W^{s, p}(\Omega),\left(1+\left|x^{J}\right|^{2}\right)^{m / 2} f \in L^{p}(\Omega)\right\},
$$

equipped with the natural norm

$$
\|f\|_{W_{m}^{s, p}(\Omega)} \equiv\|f\|_{W^{s, p}(\Omega)}+\left\|\left(1+\left|x^{J}\right|^{2}\right)^{m / 2} f\right\|_{L^{p}(\Omega)} .
$$

The space $W_{0}^{s, p}$ is then the standard Sobolev space $W^{s, p}$. For $s>d / p, W_{m}^{s, p}(\Omega)$ is an algebra, and

$$
\forall f, g \in W_{m}^{s, p}(\Omega), \quad\|f g\|_{W_{m}^{s, p}(\Omega)} \leq C(s, p, \Omega)\|f\|_{W_{m}^{s, p}(\Omega)}\|g\|_{W_{m}^{s, p}(\Omega)} .
$$

Furthermore, it follows from interpolation theory (see [10]) and Sobolev embedding that if $k \in \mathbb{N}, s>k+d / p$ and $f \in W_{m}^{s, p}(\Omega)$, then $\left(1+\left|x^{J}\right|^{\mu}\right) \nabla^{k} f \in \mathcal{C}_{b}(\Omega)$ for $0 \leq \mu<m(1-d /(p(s-k)))$. As a consequence, one has

$$
\bigcap_{s, m \in \mathbb{N}} W_{m}^{s, p}(\Omega)=\mathscr{S}(\Omega)
$$

where the Schwartz space $\mathscr{S}(\Omega)$ is defined as the set of complex valued functions $f$, smooth and such that for any $\alpha \in \mathbb{N}^{d}$ and $\mu \in \mathbb{N}$,

$$
\left(1+\left|x^{J}\right|^{\mu}\right) \partial_{x}^{\alpha} f \in \mathcal{C}_{b}(\Omega)
$$

endowed with the usual topology. The intersection (4) has to be understood with the induced topology.

The Cauchy problem for (TWI) is locally well-posed in the spaces $W_{m}^{s, p}(\Omega)$ as well as in the Schwartz space, where inverse scattering can be used. 
Proposition 1 We fix $s \in \mathbb{N}, 1 \leq p \leq \infty$ and $m \in \mathbb{N}$, with $s>d / p$. Then, for any initial data $A^{\text {in }}=$ $\left(A_{1}^{\text {in }}, A_{2}^{\text {in }}, A_{3}^{\text {in }}\right) \in W_{m}^{s, p}(\Omega)$, there exists a unique maximal solution $A=\left(A_{1}, A_{2}, A_{3}\right) \in \mathcal{C}\left(\left[0, T^{*}\right), W_{m}^{s, p}(\Omega)\right)$ to (TWI). Moreover, $T^{*}$ does not depend on s provided $s>d / p$. Finally, for some constant $c>0$ depending on $p, \Omega$ and $\left(\mathrm{v}_{\ell}, \sigma_{\ell}\right)_{1 \leq \ell \leq 3}$, there holds

$$
T^{*} \geq \frac{c}{\left\|A^{\mathrm{in}}\right\|_{W_{m}^{s, p}(\Omega)}} .
$$

Consequently, the Cauchy problem for (TWI) is locally well-posed in the Schwartz space $\mathscr{S}(\Omega)$.

Proof. We just write (TWI) under the form

$$
A(t, x)=\left(A_{\ell}(t, x)\right)_{1 \leq \ell \leq 3}=\left(A_{\ell}^{\mathrm{in}}\left(x-t \mathrm{v}_{\ell}\right)-i \sigma_{\ell} \int_{0}^{t} \bar{A}_{\ell^{\prime}} \bar{A}_{\ell^{\prime \prime}}\left(\tau, x-(t-\tau) \mathrm{v}_{\ell}\right) d \tau\right)_{1 \leq \ell \leq 3},
$$

where $\left\{\ell, \ell^{\prime}, \ell^{\prime \prime}\right\}=\{1,2,3\}$. Using the fact that $W_{m}^{s, p}(\Omega)$ is an algebra, the easy estimate

$$
\left\|\int_{0}^{t} \bar{A}_{\ell^{\prime}} \bar{A}_{\ell^{\prime \prime}}\left(\tau, x-(t-\tau) \mathrm{v}_{\ell}\right) d \tau\right\|_{W_{m}^{s, p}(\Omega)} \leq C t \sup _{0 \leq \tau \leq t}\|A(\tau)\|_{W_{m}^{s, p}(\Omega)}^{2}
$$

allows to prove by a standard fixed point argument the local well-posedness of (TWI), with the lower bound for $T^{*}$. Since $T^{*}$ does not depend on $s>d / 2$, the result in the Schwartz space follows directly from (4).

If we want to work with data having a certain decay at infinity given by some weight $\varpi: \mathbb{R}^{J} \rightarrow \mathbb{R}_{+}^{*}$ (for instance an exponential decay and $\varpi(y)=\mathrm{e}^{\gamma|y|}$ for $y \in \mathbb{R}^{J}$ and some fixed $\gamma>0$ ), we may define

$$
W_{\varpi}^{s, p}(\Omega) \equiv\left\{f \in W^{s, p}(\Omega), \varpi\left(x^{J}\right) f \in L^{p}(\Omega)\right\},
$$

endowed with the natural norm, which is also an algebra (satisfying an estimate like (3)) for $s>d / p$. The above result also holds for $W_{\varpi}^{s, p}(\Omega)$ (and if $f \in W_{\varpi}^{s, p}(\Omega), s>k+d / p$, with $s, k \in \mathbb{N}$, then $\varpi^{\mu} \nabla^{k} f \in \mathcal{C}_{b}(\Omega)$ if $0 \leq \mu<1-d /(p(s-k)))$.

\subsection{The linearized problem and stability/instability results by inverse scatter- ing}

We shall focus in the sequel on the linearized problem when $A_{1}$ and $A_{2}$ are small compared to $A_{3}$, that is

$$
\left\{\begin{array}{l}
\partial_{t} A_{1}+\mathrm{v}_{1} \cdot \nabla A_{1}+i \sigma_{1} \bar{A}_{2} \bar{A}_{3}=0 \\
\partial_{t} A_{2}+\mathrm{v}_{2} \cdot \nabla A_{2}+i \sigma_{2} \bar{A}_{3} \bar{A}_{1}=0 \\
\partial_{t} A_{3}+\mathrm{v}_{3} \cdot \nabla A_{3}=0 .
\end{array}\right.
$$

Working in the frame moving with the speed $\mathrm{v}_{3}$, replacing $A_{2}$ by $\bar{A}_{2}$ and $i \bar{A}_{3}$ by $A_{3}$, we are led to

$$
\left\{\begin{array}{l}
\partial_{t} A_{1}+v_{1} \cdot \nabla A_{1}+\sigma_{1} A_{3} A_{2}=0 \\
\partial_{t} A_{2}+v_{2} \cdot \nabla A_{2}+\sigma_{2} \bar{A}_{3} A_{1}=0,
\end{array}\right.
$$

where $\left(v_{1}, v_{2}\right) \equiv\left(\mathrm{v}_{1}-\mathrm{v}_{3}, \mathrm{v}_{2}-\mathrm{v}_{3}\right)$ and where $A_{3}=A_{3}(x)$ is a given function independent of time. The datum $A_{3}$ is called the pump-wave and (5) the pump-wave approximation. For the system (5), the Manley-Rowe identity (2) becomes

$$
\frac{d}{d t} \int_{\Omega} \sigma_{2}\left|A_{1}\right|^{2}-\sigma_{1}\left|A_{2}\right|^{2} d x=0 .
$$

This suggests that the solution $\left(A_{1}, A_{2}\right)=(0,0)$ is stable for $(5)$ if $\sigma_{1} \sigma_{2}<0$, which is what is expected from inverse scattering (see [6], [8]). Note that the inverse scattering problem for the linearized system (5) is

$$
\left\{\begin{array}{c}
i \lambda \psi_{1}+v_{1} \cdot \nabla \psi_{1}+\sigma_{1} \psi_{2} A_{3}=0 \\
i \lambda \psi_{2}+v_{2} \cdot \nabla \psi_{2}+\sigma_{2} \psi_{1} \bar{A}_{3}=0
\end{array}\right.
$$


which is exactly the system we obtain when applying Fourier transform in time for (5), with $\lambda$ the dual variable of $t$ (hence expected real). Here, we consider only bounded eigenvectors. For the inverse scattering point of view, (forward in time) stability for (5) means that (7) has no nontrivial solution with $\operatorname{Im}(\lambda)<0$ and (forward in time) instability that (7) has at least one nontrivial solution with $\operatorname{Im}(\lambda)<0$. For $\sigma_{1} \sigma_{2}>0$, the situation is more delicate. In dimension $d=1$, with $\Omega=\mathbb{R}$ at least, it is expected (see [8], [6]) that when

$$
v_{1} v_{2}<0
$$

that is if among the original speeds $\mathrm{v}_{1}, \mathrm{v}_{2}, \mathrm{v}_{3}, A_{3}$ is associated to the middle one, then if one takes $A_{3}$ as the soliton of (TWI), there is linear instability for (5). This instability leads, for the original system (TWI), to the creation of two solitons, with respective speeds $\mathrm{v}_{1}$ and $\mathrm{v}_{2}$ whereas the soliton $A_{3}$ disappears (this is called "decay instability"). This result of soliton exchange interaction is classical (see [8], [4]), and is supported by experiments and numerical simulation (see [2]). We would like to point out that the unstable eigenvalues appear in the scattering problem as zeros or poles of some holomorphic functions, and come from numerical studies or asymptotic expansions in some particular regimes. More generally, the decay instability happens each time $A_{3}$ is not too small so that the two solitons can appear. The quantities

$$
\sqrt{\frac{\sigma_{1} \sigma_{2}}{\left|v_{1} v_{2}\right|}} \times\left|\int_{\mathbb{R}} A_{3} d x\right| \quad \text { and } \quad \sqrt{\frac{\sigma_{1} \sigma_{2}}{\left|v_{1} v_{2}\right|}} \int_{\mathbb{R}}\left|A_{3}\right| d x
$$

called the area and the absolute area, are proportional to an upper bound for the possible number of solitons contained in the solution of (TWI) with initial data $\left(A_{1} \approx 0, A_{2} \approx 0, A_{3}\right)$. Thus, a smallness hypothesis

$$
\sqrt{\frac{\sigma_{1} \sigma_{2}}{\left|v_{1} v_{2}\right|}} \int_{\mathbb{R}}\left|A_{3}\right| d x<c
$$

for some absolute positive constant $c$, prevents the formation of solitons. In [4] and [8], one can find various sharp or sufficient conditions (when $\Omega=\mathbb{R}$ ) on stability in the case $\sigma_{1} \sigma_{2}>0$ and $v_{1} v_{2}<0$ (they depend on the specific regime of interest), for instance $c=\pi / 2$ is necessary and sufficient (for a real-valued function $A_{3}$, which never vanishes) in the WKB approximation, that is for a slowly varying amplitude $A_{3}$, and in the general case $c \simeq 0.903$ is sufficient. In the case

$$
v_{1} v_{2}>0
$$

this phenomenon can not occur: solitons can not be created in $A_{1}$ or $A_{2}$. However, the question of stability for (5) if $v_{1} v_{2}>0$ is then not very clear: the absence of solitons only means (by inverse scattering) that (5) has no eigenvalue in the half-space $\{\operatorname{Im}<0\}$. Let us emphasize once again that all these results hold for strongly localized in space data (notice that the condition (8) requires $A_{3} \in L^{1}(\mathbb{R})$ for example).

As already mentioned, the inverse scattering point of view give precise qualitative information, but the absence of clear functional spaces makes the statements of stability/instability results quite delicate. For the same reason, they do not give estimates for the growth of solutions in the unstable cases. Furthermore, the inverse scattering approach is not adapted to perturbation. Our aim in this paper is to determine, in each case, an $L^{p}$ or Sobolev norm in which stability or instability occurs for (5). For a given equation like (1), where the (TWI) system arises through asymptotic expansions, the resonant set is often compact (i.e. the resonant triads are bounded). Therefore, we hope that our results can help for stating and proving stability/instability results (in an adapted $L^{p}$ type space) for this original problem without inverse scattering. Indeed, since the functions involved in (TWI) will have only low frequencies, a control in any (reasonable) norm (like $L^{1}, L^{2}$ or $L^{\infty}$ norms) is acceptable.

\subsection{Statement of the results}

We first note that if $s, m \in \mathbb{N}$ are given, the initial value problem (5) is well-posed in the spaces $W_{m}^{s, p}(\Omega)$ provided $A_{3} \in W^{s, \infty}(\Omega)$. In what follows, $\Sigma_{t} A^{\text {in }}$ will denote the solution $A=\left(A_{1}, A_{2}\right)$ at time $t \geq 0$ of (5) with initial datum $A^{\text {in }}=\left(A_{1}^{\text {in }}, A_{2}^{\text {in }}\right)$. The space $L^{p}\left(\Omega, \mathbb{C}^{2}\right)$ is endowed with the norm $\left\|F=\left(F_{1}, F_{2}\right)\right\|_{L^{p}} \equiv$ $\max \left(\left\|F_{1}\right\|_{L^{p}},\left\|F_{2}\right\|_{L^{p}}\right)$.

The results for (5) below depend whether the map $A_{3}$ has only Sobolev regularity, that is $A_{3} \in W^{s, p}(\Omega)$, which is a first natural context, or $A_{3}$ is strongly localized in space $\left(A_{3} \in W_{m}^{s, p}(\Omega)\right.$ with $m$ sufficiently large), which is natural in order to use inverse scattering. The results below concern different cases which are far 
from being disjoint. It will be convenient in the following to distinguish the two cases

$\left(\mathrm{C}_{\text {per }}\right) \Lambda_{j}<\infty$ for any $1 \leq j \leq d$ such that $\left(v_{1}\right)_{j} \neq 0$ or $\left(v_{2}\right)_{j} \neq 0$,

$\left(\mathrm{C}_{\text {inf }}\right) \Lambda_{j}=\infty$ for any $1 \leq j \leq d$ such that $\left(v_{1}\right)_{j} \neq 0$ or $\left(v_{2}\right)_{j} \neq 0$,

which correspond to cases where we have periodic conditions all or none of the directions of $\operatorname{Span}\left(v_{1}, v_{2}\right)$.

The case $\sigma_{1} \sigma_{2}=0$ as well as the cases $v_{1}=0$ and/or $v_{2}=0$ are degenerate, and will be studied in the end of the section. When $v_{1}=v_{2}$, only one speed of propagation is involved and explicit computations can be carried out. Moreover, if $A_{3} \equiv 0$, then $\Sigma_{t}$ is trivially unitary in all the spaces $W^{s, p}(\Omega)$. The proofs are given in the next section.

Localized data remain localized. First, we state a result when $A_{3}$ and the initial data $A^{\text {in }}=\left(A_{1}^{\text {in }}, A_{2}^{\text {in }}\right)$ are sufficiently localized in space, then the solution $\left(A_{1}, A_{2}\right)$ of $(5)$ remains localized in space for positive times. However, since we work for large times, we have to take into account the transport terms in (5) with speeds $v_{1}$ and $v_{2}$. Therefore, we denote

$$
\Gamma \equiv\left\{\alpha_{1} v_{1}^{J}+\alpha_{2} v_{2}^{J}, \quad \alpha_{1} \geq 0, \quad \alpha_{2} \geq 0, \quad \alpha_{1}+\alpha_{2} \leq 1\right\}
$$

the convex hull of $0, v_{1}^{J}, v_{2}^{J}$ and in $\mathbb{R}^{J}$; and set for $t \geq 0$ and $x \in \Omega$

$$
D_{t}(x) \equiv \operatorname{dist}\left(x^{J}, t \Gamma\right) \text {. }
$$

Then, we have

Proposition 2 We assume $v_{1} \neq 0, v_{2} \neq 0$ and that, for some $\nu>1, A_{3}$ verifies

$$
\left(1+\left|x^{J}\right|^{\nu}\right) A_{3} \in L^{\infty}(\Omega) .
$$

Then, there exists $C>0$ and $R \geq 1$, depending only on $\sigma_{1}, \sigma_{2}, v_{1}, v_{2}, \nu$ and $\left\|\left(1+\left|x^{J}\right|^{\nu}\right) A_{3}\right\|_{\infty}$, such that for any $\mu \geq 0$ and any initial data $A^{\text {in }}=\left(A_{1}^{\text {in }}, A_{2}^{\text {in }}\right)$ such that

$$
\left(1+\left|x^{J}\right|^{\mu}\right) A^{\text {in }} \in L^{\infty}(\Omega),
$$

there holds, for $t \geq 0$,

$$
\left\|D_{t}^{\mu}(x) \Sigma_{t} A^{\mathrm{in}}\right\|_{L^{\infty}\left(\left\{D_{t} \geq R\right\}\right)} \leq C\left\|\left(1+\left|x^{J}\right|^{\mu}\right) A^{\mathrm{in}}\right\|_{L^{\infty}(\Omega)} .
$$

The stable case $\sigma_{1} \sigma_{2}<0$. From the conservation laws (6), it follows rather immediately:

Proposition 3 We assume $\sigma_{1} \sigma_{2}<0$ and fix $s \in \mathbb{N}$. If $A_{3} \in W^{s, \infty}(\Omega)$, then there exists $C>0$, depending on $A_{3}, s, \sigma_{1}, \sigma_{2}$ (but not on $v_{1}$ and $v_{2}$ ) such that for $t \geq 0$,

$$
\left\|\Sigma_{t}\right\|_{\mathscr{L}_{c}\left(H^{s}(\Omega)\right)} \leq C\left(1+t^{s}\right) .
$$

For functions depending only on time (hence in the periodic setting and with $A_{3}$ constant), the term $t^{s}$ is useless. However, it can not be removed in general, as shown by the simple example where $v_{1}=v_{2}=0$ (recall that the constant $C$ does not depend on the speeds $v_{1}$ and $v_{2}$ ), in which case (5) becomes

$$
\left\{\begin{array}{l}
\partial_{t} A_{1}+\sigma_{1} A_{2} A_{3}=0 \\
\partial_{t} A_{2}+\sigma_{2} \bar{A}_{3} A_{1}=0,
\end{array}\right.
$$

thus

$$
A_{1}(t, x)=\alpha(x) \cos \left(t \sqrt{\left|\sigma_{1} \sigma_{2}\right|}\left|A_{3}\right|(x)\right)+\beta(x) \sin \left(t \sqrt{\left|\sigma_{1} \sigma_{2}\right|}\left|A_{3}\right|(x)\right)
$$

and the estimate of Proposition 3 is optimal. Nevertheless, the case $v_{1}=v_{2}=0$ is very degenerate, since all the speeds are equal. However, in dimension $d \geq 3$, we can argue analogously in case $\left(\mathrm{C}_{\text {per }}\right)$ with functions $\left(A_{1}, A_{2}\right)$ depending only on coordinates orthogonal to $\operatorname{Span}\left(v_{1}, v_{2}\right)$ (and compactly supported) to deduce the optimality of the estimate in Proposition 3. This is also possible if $d=2$ and the speeds $v_{1}, v_{2}$ are collinear (still in case $\left(\mathrm{C}_{\mathrm{per}}\right)$ ). The secular term $t^{s}$ means that in general, oscillations in time are expected. We do not know whether the estimate of Proposition 3 is optimal in the other cases for $d \geq 2$. However, in the one dimensional case, one can always improve the above result by showing stability in all the spaces $H^{s}(\Omega)$, provided the speeds $v_{1}$ and $v_{2}$ are nonzero. 
Proposition 4 Let $d=1$, fix $s \in \mathbb{N}$ and assume that $\sigma_{1} \sigma_{2}<0$, that $A_{3} \in W^{s, \infty}(\Omega)$ and that $v_{1} \neq 0$ and $v_{2} \neq 0$. Then, there exists $C>0$, depending on $s, A_{3}, \Omega, v_{1}, v_{2}, \sigma_{1}$ and $\sigma_{2}$, such that, for $t \geq 0$,

$$
\left\|\Sigma_{t}\right\|_{\mathscr{L}_{c}\left(H^{s}(\Omega)\right)} \leq C .
$$

In the above Proposition, the constant $C$ depends on $A_{3}$ through some eigenvalue problem. It is clear from these results that when $\sigma_{1} \sigma_{2}<0$, the scattering problem (7) has, as expected, no eigenvalue in $\{\operatorname{Im}<0\}$ if we require the corresponding eigenvector to belong to $L^{2}(\Omega)$.

The case of small and localized data. We now consider strongly localized in space data, with a smallness assumption, in the spirit of (8). Then, stability is expected for (5). Note however that the result below is not restricted to the unstable situation $\sigma_{1} \sigma_{2}>0$. The hypothesis below on $A_{3}$ are natural when one writes (5) under the Duhamel form

$$
\left\{\begin{array}{l}
A_{1}(t, x)=A_{1}^{\mathrm{in}}\left(x-t v_{1}\right)-\sigma_{1} \int_{0}^{t} A_{2}\left(\tau, x-(t-\tau) v_{1}\right) A_{3}\left(x-(t-\tau) v_{1}\right) d \tau \\
A_{2}(t, x)=A_{2}^{\mathrm{in}}\left(x-t v_{2}\right)-\sigma_{2} \int_{0}^{t} A_{1}\left(\tau, x-(t-\tau) v_{2}\right) \bar{A}_{3}\left(x-(t-\tau) v_{2}\right) d \tau .
\end{array}\right.
$$

Proposition 5 We assume $v_{1} \neq 0, v_{2} \neq 0$ and consider the case $\left(\mathrm{C}_{\mathrm{inf}}\right)$. We fix $s \in \mathbb{N}$ and we assume that $A_{3} \in \mathcal{C}_{b}^{s}(\Omega)$ is such that

$$
\forall \alpha \in \mathbb{N}^{d}, \quad|\alpha| \leq s, \quad \sup _{x \in \Omega}\left\{\int_{x+\mathbb{R} v_{1}}\left|\partial_{x}^{\alpha} A_{3}\right|+\int_{x+\mathbb{R} v_{2}}\left|\partial_{x}^{\alpha} A_{3}\right|\right\}<\infty
$$

and satisfies the smallness assumption

$$
\frac{\left|\sigma_{1} \sigma_{2}\right|}{\left|v_{1}\right| \cdot\left|v_{2}\right|}\left(\sup _{x \in \Omega} \int_{x+\mathbb{R} v_{1}}\left|A_{3}\right|\right)\left(\sup _{x \in \Omega} \int_{x+\mathbb{R} v_{2}}\left|A_{3}\right|\right)<1
$$

Then, there exists some constant $C$ depending only on $s, \sigma_{1}, \sigma_{2}, v_{1}, v_{2}, A_{3}$ and (11) such that for $t \geq 0$,

$$
\left\|\Sigma_{t}\right\|_{\mathscr{L}_{c}\left(W^{s, \infty}(\Omega)\right)} \leq C .
$$

We may notice that in dimension $d=1$, with $\sigma_{1} \sigma_{2}>0$ and $v_{1} v_{2}<0$, our smallness assumption (11) is

$$
\sqrt{\frac{\sigma_{1} \sigma_{2}}{\left|v_{1} v_{2}\right|}} \int_{\mathbb{R}}\left|A_{3}\right| d x<1
$$

which is an upper bound like (8) with $c=1$, which is better than the (sufficient) value (in the general case) $c \simeq 0.903$, but worse than the "exact" value $c=\pi / 2$ for the WKB approximation. Here again, the above result shows that (7) has no eigenvalue in $\{\operatorname{Im}<0\}$ when $A_{3}$ is both localized and small. In dimension $d=1$, (11) requires $A_{3}$ to be in $L^{1}$, and we would like to point out that for (1) (for (KdV) or (KP) equations, for instance), integrability conditions can be imposed on $u$ in order to have finite mass $\int_{\mathbb{R}} u d x$ (which is often a formally conserved quantity).

The case $\boldsymbol{\sigma}_{\mathbf{1}} \boldsymbol{\sigma}_{\mathbf{2}}>\mathbf{0}$. In the case $\sigma_{1} \sigma_{2}>0$, we first give an estimate of exponential growth.

Proposition 6 We assume $\sigma_{1} \sigma_{2}>0$, fix $s \in \mathbb{N}$ and let $A_{3} \in W^{s, \infty}(\Omega), A_{3} \not \equiv 0$. We define

$$
\gamma \equiv \sqrt{\sigma_{1} \sigma_{2}}\left\|A_{3}\right\|_{L^{\infty}(\Omega)}>0
$$

Then, for any $1 \leq p \leq \infty$, there exists $C>0$, depending on $A_{3}, p, s, \sigma_{1}$ and $\sigma_{2}$ (but not on $v_{1}, v_{2}$ ) such that for any $t \geq 0$

$$
\left\|\Sigma_{t}\right\|_{\mathscr{L}_{c}\left(W^{s, p}(\Omega)\right)} \leq C\left(1+t^{s}\right) \mathrm{e}^{\gamma t} .
$$

We can notice (as in the analogous case discussed just after Proposition 3 ) that the polynomial growth in $t^{s}$ can be achieved with either $v_{1}=v_{2}=0$, either $d \geq 3$, either $d=2$ and $v_{1}, v_{2}$ collinear.

In the case $\left(\mathrm{C}_{\mathrm{per}}\right)$, the estimate in Proposition 6 is almost optimal, as shown by the following 
Proposition 7 We assume $\sigma_{1} \sigma_{2}>0$ and consider the case $\left(\mathrm{C}_{\mathrm{per}}\right)$, with $A_{3} \in L^{\infty}(\Omega), A_{3} \not \equiv 0$, and let

$$
\gamma \equiv \sqrt{\sigma_{1} \sigma_{2}}\left\|A_{3}\right\|_{L^{\infty}(\Omega)}>0
$$

(i) If $\Lambda_{j}<\infty$ for every $1 \leq j \leq d$ and $A_{3}$ is independent of $x$, then $-i \gamma \in i \mathbb{R}_{-}^{*} \subset\{\operatorname{Im}<0\}$ is an eigenvalue for (7) with multiplicity one among the functions constant in space.

(ii) If $d>\operatorname{dim} \operatorname{Span}\left(v_{1}, v_{2}\right)$ and $A_{3} \in \mathcal{C}_{b}(\Omega)$ depends only on the coordinates orthogonal to $\operatorname{Span}\left(v_{1}, v_{2}\right)$, then for any $0<\varepsilon<\gamma$, there exist a ball $B_{2 r_{\varepsilon}}\left(y_{\varepsilon}\right)$ and initial data $A^{\text {in }}=\left(A_{1}^{\text {in }}, A_{2}^{\text {in }}\right) \in \mathcal{C}_{c}^{\infty}\left(B_{2 r_{\varepsilon}}\left(y_{\varepsilon}\right)\right)$ such that $\left|A^{\mathrm{in}}\right|_{\infty} \leq 1$ in $B_{2 r_{\varepsilon}}\left(y_{\varepsilon}\right),\left|A^{\mathrm{in}}\right|_{\infty}=1$ in $B_{r_{\varepsilon}}\left(y_{\varepsilon}\right)$ and

$$
\left|\Sigma_{t} A^{\text {in }}\right|_{\infty} \geq \mathrm{e}^{(\gamma-\varepsilon) t} \quad \text { in } \quad B_{r_{\varepsilon}}\left(y_{\varepsilon}\right)
$$

(iii) If $d>\operatorname{dim} \operatorname{Span}\left(v_{1}, v_{2}\right)$ and $A_{3}$ depends only on the coordinates orthogonal to $\operatorname{Span}\left(v_{1}, v_{2}\right)$ and is such that, for some ball $B_{2 r}(y)$, we have $\left|A_{3}\right|(x)=\left\|A_{3}\right\|_{L^{\infty}(\Omega)}$ for $x \in B_{2 r}(y)$, then $-i \gamma \in i \mathbb{R}_{-}^{*} \subset\{\operatorname{Im}<0\}$ is an eigenvalue for (7) with infinite multiplicity.

(iv) If $1 \leq p \leq \infty, d=1, A_{3}$ is real-valued and $v_{1}=v_{2} \neq 0$, then there exists some constant $C>0$, depending on $\sigma_{1}, \sigma_{2}$ and $v_{1}=v_{2}$ such that if $t \geq 0$, then

$$
\left\|\Sigma_{t}\right\|_{\mathscr{L}_{c}\left(L^{p}(\Omega)\right)} \leq C \exp \left(t\left|\frac{\sqrt{\sigma_{1} \sigma_{2}}}{|\Omega|} \int_{\Omega} A_{3}(y) d y\right|\right) .
$$

Moreover, the eigenvalues for (7) (with multiplicity) are the

$$
\frac{m v_{1}}{\Lambda} \pm i\left|\frac{\sqrt{\sigma_{1} \sigma_{2}}}{|\Omega|} \int_{\Omega} A_{3}(y) d y\right|, \quad \text { for } \quad m \in \mathbb{Z}
$$

In particular, they are simple as soon as $\int_{\Omega} A_{3}(y) d y \neq 0$.

We notice that in case $(i i)$, there holds, for any $1 \leq p \leq \infty$ and $t \geq 0$,

$$
\left\|\Sigma_{t} A^{\mathrm{in}}\right\|_{L^{p}(\Omega)} \geq C_{p}\left\|A^{\mathrm{in}}\right\|_{L^{p}(\Omega)} \mathrm{e}^{(\gamma-\varepsilon) t},
$$

where the constant $C_{p}$ depends only on $p$ (and $A_{3}, \sigma_{1}, \sigma_{2}$ and $d$ ) and not on $0<\varepsilon<\gamma$. Consequently,

$$
\left\|\Sigma_{t}\right\|_{\mathscr{L}_{c}\left(L^{p}(\mathbb{R})\right)} \geq C_{p} \mathrm{e}^{(\gamma-\varepsilon) t} .
$$

Furthermore, for $(i i)$ and (iii), if $d=\operatorname{dim} \operatorname{Span}\left(v_{1}, v_{2}\right)$, then we are in case $(i)$.

We now turn to the case $\left(\mathrm{C}_{\text {inf }}\right)$. By Proposition 5, we now that stability holds in $W^{s, \infty}$ if $A_{3}$ is suitably small. In dimension $d=1$ and without smallness assumption on $A_{3}$, we can show that (7) may have some eigenvalues in $\{\operatorname{Im}<0\}$.

Proposition 8 We assume $\Omega=\mathbb{R}\left(d=1, \Lambda_{1}=+\infty\right), \sigma_{1} \sigma_{2}>0$ and $v_{1} v_{2}<0$ (we are then in case $\left.\left(\mathrm{C}_{\mathrm{inf}}\right)\right)$. For $A_{3} \in L^{\infty}(\mathbb{R})$, we denote

$$
\gamma_{*} \equiv 2 \sqrt{\sigma_{1} \sigma_{2}} \frac{\sqrt{\left|v_{1} v_{2}\right|}}{\left|v_{1}-v_{2}\right|}\left\|A_{3}\right\|_{L^{\infty}(\mathbb{R})} \in\left(0, \sqrt{\sigma_{1} \sigma_{2}}\left\|A_{3}\right\|_{L^{\infty}(\mathbb{R})}\right] .
$$

(i) Assume $A_{3}=\mathcal{A}_{3} \mathbf{1}_{[a, b]}$, with $\mathcal{A}_{3} \in \mathbb{C}^{*}$ and $-\infty<a<b<+\infty$. Then, if

$$
\frac{\sqrt{\sigma_{1} \sigma_{2}}(b-a)\left|\mathcal{A}_{3}\right|}{\sqrt{\left|v_{1} v_{2}\right|}}=\frac{\sqrt{\sigma_{1} \sigma_{2}}}{\sqrt{\left|v_{1} v_{2}\right|} \mid} \int_{\mathbb{R}} A_{3}(x) d x \mid \leq \pi / 2
$$

(7) has no eigenvalue in $\{\operatorname{Im}<0\}$, and if $m \in \mathbb{N}^{*}$ is such that

$$
\frac{(2 m-1) \pi}{2}<\frac{\sqrt{\sigma_{1} \sigma_{2}}(b-a)\left|\mathcal{A}_{3}\right|}{\sqrt{\left|v_{1} v_{2}\right|}}=\frac{\sqrt{\sigma_{1} \sigma_{2}}}{\sqrt{\left|v_{1} v_{2}\right|}}\left|\int_{\mathbb{R}} A_{3}(x) d x\right| \leq \frac{(2 m+1) \pi}{2},
$$


then $(7)$ has exactly $m$ eigenvalues in $\{\operatorname{Im}<0\}$, which are simple and lie in $\left(0,-i \gamma_{*}\right)$.

(ii) We assume that $A_{3} \in L^{1} \cap L^{\infty}(\mathbb{R})$ is real-valued and satisfies

$$
\sqrt{\frac{\sigma_{1} \sigma_{2}}{\left|v_{1} v_{2}\right|}} \times\left|\int_{\mathbb{R}} A_{3}(x) d x\right|>\frac{\pi}{2} .
$$

Then, there exists at least one eigenvalue $\lambda_{0} \in i \mathbb{R}_{-}^{*} \subset\{\operatorname{Im}<0\}$ for $(7)$, with corresponding eigenvector decaying exponentially fast at infinity. Hence, for any $1 \leq p \leq \infty$, instability holds for (5) in $L^{p}(\mathbb{R})$.

(iii) If $A_{3} \in L^{1} \cap L^{\infty}(\mathbb{R})$, then there exists a constant $C$, depending on $\sigma_{1}, \sigma_{2}, v_{1}, v_{2}$ and $A_{3}$, such that, for any $t \geq 0$,

$$
\left\|\Sigma_{t}\right\|_{\mathscr{L}_{c}\left(L^{\infty}(\mathbb{R})\right)} \leq C \mathrm{e}^{\gamma_{*} t} .
$$

Remark 1 In $(i)$, by an appropriate choice of the area of $A_{3}$, we can make the eigenvalues as close as we want to $-i \gamma_{*}$, so that the estimate in (iii) is (in general) optimal. We emphasize that, comparing with Proposition 7, the optimal rate $\gamma_{*}$ in (iii) does depend on the speeds $v_{1}$ and $v_{2}$. In (iii), if $v_{1}+v_{2}=0$, then $2 \frac{\sqrt{\left|v_{1} v_{2}\right|}}{\left|v_{1}-v_{2}\right|}=1$, thus $\gamma=\gamma_{*}$ and Proposition 6 gives the same estimate in all $L^{p}(\mathbb{R})$ spaces without assuming $A_{3} \in L^{1}(\mathbb{R})$. The case $(i i)$ is consistent with the (necessary and) sufficient condition (for realvalued everywhere nonzero functions $A_{3}$ ) in the WKB approximation $c=\pi / 2$ (however, on the one hand, we work neither in a WKB approximation, neither with a constraint on the constant sign of $A_{3}$; and on the other hand, in the WKB approximation, the condition is also sufficient). In any case, if $d=1, \sigma_{1} \sigma_{2}>0$ and $v_{1} v_{2}<0$, the soliton is linearly unstable since it is in $L^{1}(\mathbb{R})$ and has area equal to $\pi>\pi / 2$. Let us mention that we have no result corresponding to $(i i)$ when $A_{3}$ is complex-valued. Of course, the results of Propositions 5 and 8 leave open, when $d=1$ and $\sigma_{1} \sigma_{2}>0>v_{1} v_{2}$, the case where $A_{3}$ is real-valued with

$$
\sqrt{\frac{\sigma_{1} \sigma_{2}}{\left|v_{1} v_{2}\right|}} \times \int_{\mathbb{R}}\left|A_{3}\right|(x) d x \geq 1 \quad \text { but } \quad \sqrt{\frac{\sigma_{1} \sigma_{2}}{\left|v_{1} v_{2}\right|}} \times\left|\int_{\mathbb{R}} A_{3}(x) d x\right| \leq \frac{\pi}{2} .
$$

A numerical example is given in [8] where $A_{3}$ is the characteristic function of an interval with corresponding area between 1 and $\pi / 2$ : for (TWI), there is instability and blow-up in finite time (this suggests instability for (5), but $(i)$ shows that there is no eigenvalue for $(7)$ in $\{\operatorname{Im}<0\}$ ). We have not been able to prove any estimate for the solution of (5) better than the exponential growth given in (iii) when restricting ourselves to energy methods. Even though we could locate precisely the eigenvalues in $\{\operatorname{Im}<0\}$, using this information to estimate the semigroup seems a difficult task.

In the case $\Omega=\mathbb{R}$ and $v_{1} v_{2}>0$, we do not expect eigenvalues in $\{\operatorname{Im}<0\}$ for (7). However, the stability for (5) is linked to some integrability conditions on $A_{3}$.

Proposition 9 We assume $\Omega=\mathbb{R}\left(d=1, \Lambda_{1}=+\infty\right), \sigma_{1} \sigma_{2}>0$ and $v_{1} v_{2}>0$ (we are then in case $\left(\mathrm{C}_{\mathrm{inf}}\right)$ ). (i) We assume $A_{3} \in L_{\text {loc }}^{1}(\mathbb{R})$ real-valued, $v_{1}=v_{2} \neq 0$, and fix $1 \leq p \leq \infty$. Then stability holds in $L^{p}(\mathbb{R})$ for (5) if and only if

$$
\sup _{-\infty<a<b<+\infty}\left|\int_{a}^{b} A_{3}(y) d y\right|<\infty
$$

in which case there exists $C_{p}$, depending only on $p, \sigma_{1}$ and $\sigma_{2}$, such that for any $t \geq 0$,

$$
\left\|\Sigma_{t}\right\|_{\mathscr{L}_{c}\left(L^{p}(\mathbb{R})\right)} \leq C_{p} \exp \left(\frac{\sqrt{\sigma_{1} \sigma_{2}}}{\left|v_{1}\right|} \sup _{-\infty<a<b<+\infty}\left|\int_{a}^{b} A_{3}(y) d y\right|\right) .
$$

If (12) is not true, then $\left\|\Sigma_{t}\right\|_{\mathscr{L}_{c}\left(L^{p}(\mathbb{R})\right)} \rightarrow+\infty$ as $t \rightarrow+\infty$ and there exists $C_{p}>1$, depending only on $p, \sigma_{1}$ and $\sigma_{2}$, such that for any $t \geq 0$,

$$
\frac{1}{C_{p}} \exp \left(\frac{\sqrt{\sigma_{1} \sigma_{2}}}{\left|v_{1}\right|} \sup _{x \in \mathbb{R}}\left|\int_{x-t\left|v_{1}\right|}^{x} A_{3}(y) d y\right|\right) \leq\left\|\Sigma_{t}\right\|_{\mathscr{L}_{c}\left(L^{p}(\mathbb{R})\right)} \leq C_{p} \exp \left(\frac{\sqrt{\sigma_{1} \sigma_{2}}}{\left|v_{1}\right|} \sup _{x \in \mathbb{R}}\left|\int_{x-t\left|v_{1}\right|}^{x} A_{3}(y) d y\right|\right) .
$$

(ii) There exists a constant $C$, depending only on $\sigma_{1}, \sigma_{2}, v_{1}$ and $v_{2}$ such that, if $A_{3} \in L_{\text {loc }}^{1}(\mathbb{R})$ and $t \geq 0$, we have

$$
\left\|\Sigma_{t}\right\|_{\mathscr{L}_{c}\left(\mathcal{C}_{b}(\mathbb{R})\right)} \leq C \exp \left(\sqrt{\frac{\sigma_{1} \sigma_{2}}{v_{1} v_{2}}} \sup _{x \in \mathbb{R}} \int_{x-t \max \left(\left|v_{1}\right|,\left|v_{2}\right|\right)}^{x}\left|A_{3}\right|(y) d y\right)
$$


In particular, if $A_{3} \in L^{1}(\mathbb{R})$, then stability holds in $\mathcal{C}_{b}(\mathbb{R})$ for (5) and, for $t \geq 0$,

$$
\left\|\Sigma_{t}\right\|_{\mathscr{L}_{c}\left(\mathcal{C}_{b}(\mathbb{R})\right)} \leq C \exp \left(\sqrt{\frac{\sigma_{1} \sigma_{2}}{v_{1} v_{2}}}\left\|A_{3}\right\|_{L^{1}(\mathbb{R})}\right)
$$

(iii) We assume $e^{1}$ that $v_{1}>0$ and $v_{2}>0$, that $A_{3} \in \mathcal{C}(\mathbb{R})$ is real-valued, $A_{3} \notin L^{1}(\mathbb{R})$, and satisfies ${ }^{2}$

$$
A_{3}=0 \quad \text { in }[1,+\infty), \quad-\sigma_{1} A_{3} \geq 0 \quad \text { in } \mathbb{R}, \quad-\sigma_{1} A_{3} \text { is nondecreasing in } \mathbb{R}^{-} .
$$

Then, $\left\|\Sigma_{t}\right\|_{\mathscr{L}_{c}\left(\mathcal{C}_{b}(\mathbb{R})\right)} \rightarrow+\infty$ as $t \rightarrow+\infty$. More precisely, there exists a constant $c>1$ depending only on $\sigma_{1}$, $\sigma_{2}, v_{1}$ and $v_{2}$ such that, for $t \geq 0$,

$$
\left\|\Sigma_{t}\right\|_{\mathscr{L}_{c}\left(L^{\infty}(\mathbb{R})\right)} \geq c \exp \left(\frac{\min \left(v_{1}, v_{2}\right)}{\max \left(v_{1}, v_{2}\right)} \sqrt{\frac{\sigma_{1} \sigma_{2}}{v_{1} v_{2}}} \sup _{x \in \mathbb{R}}\left|\int_{x-t \max \left(v_{1}, v_{2}\right)}^{x} A_{3}(y) d y\right|\right) .
$$

Remark 2 In view of the right-hand side part of the estimate in (ii), problem (7) has no eigenvalue in $\{\operatorname{Im}<0\}$ (for eigenvectors in $L^{\infty}(\mathbb{R})$ ) if $A_{3} \in L^{2}(\mathbb{R})$. However, instability can occur for (5).

In [9] (section 4), the question of the stability for (5) was studied (for $d=1$ ) in the case $v_{1}=v_{2}$ : stability was suggested when $A_{3} \in L^{1}(\mathbb{R})$ and instability was shown by an explicit example for some $A_{3} \notin L^{1}(\mathbb{R})$. This led G. Schneider to the conjecture that stability holds for (5) (if $d=1$ and $\sigma_{1} \sigma_{2}>0$ ) for sufficiently localized functions $A_{3}$ (at least in $L^{1}(\mathbb{R})$ ) and instability occurs for nonlocalized functions $A_{3}$. As we see from the results obtained by inverse scattering (subsection 1.2) and the above results, the situation is more complex.

If $\sigma_{1} \sigma_{2}>0$ and $v_{1}=v_{2}=0,(5)$ reduces to (9), with solutions that can be written explicitly:

$$
\Sigma_{t}=\exp \left(-t\left(\begin{array}{cc}
0 & \sigma_{1} A_{3}(x) \\
\sigma_{2} \bar{A}_{3}(x) & 0
\end{array}\right)\right)
$$

and the matrix $\Sigma_{t}$ has eigenvalues $\exp \left( \pm t \sqrt{\sigma_{1} \sigma_{2}}\left|A_{3}\right|(x)\right)$ hence, the estimates for $\Sigma_{t}$ are as in Proposition 7 . If $A_{3} \in \mathcal{C}^{2}(\Omega)$, notice that in this simple case, $-i \sqrt{\sigma_{1} \sigma_{2}}\left\|A_{3}\right\|_{L^{\infty}(\Omega)} \in\{\operatorname{Im}<0\}$ is not an eigenvalue for (7) as soon as $\left\|A_{3}\right\|_{L^{\infty}(\Omega)}$ is a non-degenerate critical value of $\left|A_{3}\right|$. In dimension $d=1$, it remains to study the degenerate case where one speed $v_{1}$ or $v_{2}$ is zero. Possibly changing $x$ for $-x$ and exchanging the indices 1 and 2 , we do not loose generality assuming $v_{1}=0<v_{2}$.

Proposition 10 We assume $\Omega=\mathbb{R}\left(d=1, \Lambda_{1}=+\infty\right), \sigma_{1} \sigma_{2}>0$ and $v_{1}=0<v_{2}$ (we are then in case $\left.\left(\mathrm{C}_{\mathrm{inf}}\right)\right)$.

(i) Assume that $A_{3} \in L_{\text {loc }}^{\infty}(\mathbb{R})$ is such that $\left|A_{3}\right| \geq \mathcal{A}_{3}>0$ on an interval $I$ of length $\ell>0$. Then, for any $0<\varepsilon<\ell$, there exists a constant $c_{\varepsilon}>0$, depending on $\varepsilon$ and $\ell$, such that

$$
\left\|\Sigma_{t}\right\|_{\mathscr{L}_{c}(L \infty(\mathbb{R}))} \geq c_{\varepsilon} \exp \left(\sqrt{\frac{\sigma_{1} \sigma_{2}}{v_{2}}} \mathcal{A}_{3} \sqrt{(\ell-\varepsilon) t}\right) .
$$

(ii) Assume that $A_{3} \in L^{\infty}(\mathbb{R})$ is compactly supported in an interval $I$ of length $\ell>0$. Then, there exists a constant $C>0$, depending on $\ell, \sigma_{1}, \sigma_{2}$ and $v_{2}$, such that for any $t \geq 0$,

$$
\left\|\Sigma_{t}\right\|_{\mathscr{L}_{c}\left(L^{\infty}(\mathbb{R})\right)} \leq C \exp \left(\sqrt{\frac{\sigma_{1} \sigma_{2}}{v_{2}}}\left\|A_{3}\right\|_{L^{\infty}(\mathbb{R})} \sqrt{\ell t}\right) .
$$

Let us mention that if $A_{3}=\mathcal{A}_{3} \mathbf{1}_{[0, \ell]}$, with $\mathcal{A}_{3} \in \mathbb{C}^{*}$, then estimates in $(i)$ and (ii) give the same upper and lower bounds in the limit $\varepsilon \rightarrow 0$.

We now turn (in the case $\left(\mathrm{C}_{\text {inf }}\right)$ ), to the higher dimensional situation.

\footnotetext{
${ }^{1}$ Otherwise, change $x$ for $-x$.

${ }^{2}$ We can choose a smooth function $A_{3}$ such that $A_{3}(x)=\sigma_{1}(1-x)^{\nu}$ for $x \leq 0$, with $\nu \in(1 / 2,1)$, for instance, and $A_{3} \in H^{s}(\mathbb{R})$ for every $s \in \mathbb{N}$.
} 
Proposition 11 We assume $\Omega=\mathbb{R}^{2}\left(d=2, \Lambda_{1}=\Lambda_{2}=\infty\right), \sigma_{1} \sigma_{2}>0$ and that $v_{1}, v_{2}$ are not collinear (we are then in case $\left(\mathrm{C}_{\mathrm{inf}}\right)$ ). We assume that $A_{3}$ satisfies, for some $M \geq 0$ and $\nu>1$, the decay estimate

$$
\forall x \in \mathbb{R}^{2}, \quad\left|A_{3}\right|(x) \leq \frac{M}{1+|x|_{\infty}^{\nu}} .
$$

Then, there exists a constant $C(\nu, M)$, depending on $M, \nu, \sigma_{1}, \sigma_{2}, v_{1}$ and $v_{2}$ such that, for any $t \geq 0$,

$$
\left\|\Sigma_{t}\right\|_{\mathscr{L}_{c}\left(\mathcal{C}_{b}\left(\mathbb{R}^{2}\right)\right)} \leq C(\nu, M) .
$$

If $v_{1}$ and $v_{2}$ are collinear, we can reduce to one of the cases of the one-dimensional situation, with the extra-variables as simple parameters. This is also what happens if $d \geq 3$ and $v_{1}$ and $v_{2}$ are not collinear.

The degenerate case $\boldsymbol{\sigma}_{\mathbf{1}} \boldsymbol{\sigma}_{\mathbf{2}}=\mathbf{0}$. We now focus on the degenerate case $\sigma_{1} \sigma_{2}=0$. First, if $\sigma_{1}=\sigma_{2}=0$, (5) reduces to two decoupled free transport equations with explicit solutions and conservation of all $W^{s, p}(\Omega)$ norms. If $\sigma_{2}=0 \neq \sigma_{1}$, the function $A_{2}$ can be computed explicitly and we obtain from (10)

$$
A_{2}(t, x)=A_{2}^{\mathrm{in}}\left(x-t v_{2}\right), \quad A_{1}(t, x)=A_{1}^{\mathrm{in}}\left(x-t v_{1}\right)-\sigma_{1} \int_{0}^{t} A_{3}\left(x-(t-\tau) v_{1}\right) A_{2}^{\mathrm{in}}\left(x-(t-\tau) v_{1}-\tau v_{2}\right) d \tau,
$$

for which one infers easily the following estimates:

- if $v_{1} \neq 0$ and $v_{1} \neq v_{2}$, there exists $C$, depending on $\sigma_{1}, v_{1}$ and $v_{2}$ such that

$$
\left\|\Sigma_{t} A^{\mathrm{in}}\right\|_{L^{\infty}(\Omega) \times L^{2}(\Omega)} \leq C\left[\left\|A^{\mathrm{in}}\right\|_{L^{\infty}(\Omega) \times L^{2}(\Omega)}+\left(\sup _{x \in \Omega} \int_{x+\mathbb{R} v_{1}}\left|A_{3}\right|^{2}\right)^{\frac{1}{2}}\left(\sup _{x \in \Omega} \int_{x+\mathbb{R}\left(v_{1}-v_{2}\right)}\left|A_{2}^{\mathrm{in}}\right|^{2}\right)^{\frac{1}{2}}\right]
$$

and

$$
\left\|\Sigma_{t}\right\|_{\mathscr{L}_{c}\left(L^{\infty}(\Omega)\right)} \leq C\left(1+\sup _{x \in \Omega} \int_{x+\mathbb{R} v_{1}}\left|A_{3}\right|\right)
$$

provided the supremum in the right-hand side is finite, and otherwise

$$
\left\|\Sigma_{t}\right\|_{\mathscr{L}_{c}\left(L^{\infty}(\Omega)\right)} \approx\left|\sigma_{1}\right| \sup _{x \in \Omega} \int_{x-[0, t] v_{1}}\left|A_{3}\right| \quad \text { as } \quad t \rightarrow+\infty .
$$

Thus, in particular, if $d=1$ and $A_{3} \in L^{2}(\mathbb{R})$ but $A_{3} \notin L^{1}(\mathbb{R})$, then stability holds for $(5)$ in $L^{\infty}(\mathbb{R}) \times L^{2}(\mathbb{R})$ but not in $L^{\infty}(\mathbb{R})$.

- if $v_{1}=v_{2}=0$, then for any $1 \leq p \leq \infty$,

$$
\left\|\Sigma_{t}\right\|_{\mathscr{L}_{c}\left(L^{p}(\Omega)\right)} \approx t\left\|A_{3}\right\|_{L^{\infty}(\Omega)} \quad \text { as } \quad t \rightarrow+\infty ;
$$

- if $v_{1}=v_{2} \neq 0$, then, there exists $C$, depending on $\sigma_{1}$ and $v_{1}=v_{2}$ such that, for $t \geq 0$,

$$
\left\|\Sigma_{t}\right\|_{\mathscr{L}_{c}\left(L^{p}(\Omega)\right)} \leq C\left(1+\frac{\left|\sigma_{1}\right|}{\left|v_{1}\right|} \sup _{x \in \Omega, \tau \geq 0}\left|\int_{x-[0, \tau] v_{1}} A_{3}\right|\right)
$$

(if the supremum in the right-hand side is finite), and otherwise

$$
\left\|\Sigma_{t}\right\|_{\mathcal{L}_{c}\left(L^{p}(\Omega)\right)} \approx \frac{\left|\sigma_{1}\right|}{\left|v_{1}\right|} \sup _{x \in \Omega}\left|\int_{x-[0, t] v_{1}} A_{3}\right| \quad \text { as } \quad t \rightarrow+\infty ;
$$

- if $v_{1}=0 \neq v_{2}$, then

$$
A_{1}(t, x)=A_{1}^{\mathrm{in}}(x)-\sigma_{1} A_{3}(x) \int_{x-[0, t] v_{2}} A_{2}^{\mathrm{in}},
$$

and the situation is very degenerate. We can state, for instance, that if $d=1$,

$$
\left\|\Sigma_{t}\right\|_{\mathscr{L}_{c}\left(L^{\infty}(\Omega) \times L^{1}(\Omega)\right)} \leq\left(1+\left|\sigma_{1}\right|\left\|A_{3}\right\|_{L^{\infty}(\Omega)}\right)
$$

and for $d \geq 2$, denoting

$$
\|f\|_{L_{\perp}^{\infty} L_{v_{2}}^{1}(\Omega)} \equiv \sup _{x \in \Omega} \int_{x+\mathbb{R} v_{2}}|f|
$$


we have

$$
\left\|\Sigma_{t}\right\|_{L^{\infty}(\Omega) \times L_{\perp}^{\infty} L_{v_{2}}^{1}(\Omega)} \leq\left(1+\left|\sigma_{1}\right|\left\|A_{3}\right\|_{L^{\infty}(\Omega)}\right) .
$$

The paper [9] (see also other papers by the same author) investigates, for a nonlinear wave equation in dimension one in the spirit of (1), the problem of the approximation of a wavetrain by the nonlinear Schrödinger equation. The case studied corresponds to the stable one $\sigma_{1} \sigma_{2}<0$. In [4], we shall study this problem (in arbitrary dimension, replacing the nonlinear Schrödinger equation by the Davey-Stewartson system if $d \geq 2$ ) for a general semilinear equation, taking into account all possible cases and using the above semigroup estimates.

\section{Proofs of the results}

In the sequel, $A=\left(A_{1}, A_{2}\right)$ always stands for $\Sigma_{t}\left(A_{1}^{\text {in }}, A_{2}^{\text {in }}\right)=\Sigma_{t} A^{\text {in }}$. Unless otherwise stated, the $L^{p}$ norms are taken in the space variable.

\subsection{Proof of Proposition 2}

Let $x \in \Omega$ with $D_{t}(x) \geq R \geq 1$. Then, from (10), it comes

$$
\begin{aligned}
D_{t}^{\mu}(x)\left|A_{1}\right|(t, x) & \leq D_{t}^{\mu}(x)\left|A_{1}^{\mathrm{in}}\right|\left(x-t v_{1}\right)+\left|\sigma_{1}\right| \int_{0}^{t} D_{t}^{\mu}(x)\left|A_{2}\right|\left(\tau, x-(t-\tau) v_{1}\right)\left|A_{3}\right|\left(x-(t-\tau) v_{1}\right) d \tau \\
& \leq\left|x-t v_{1}\right|^{\mu}\left|A_{1}^{\mathrm{in}}\right|\left(x-t v_{1}\right)+\left|\sigma_{1}\right| \int_{0}^{t} a_{2}(\tau)\left|A_{3}\right|\left(x-(t-\tau) v_{1}\right) d \tau
\end{aligned}
$$

with

$$
a_{\ell}(\tau) \equiv\left\|D_{\tau}^{\mu}(\cdot) A_{\ell}(\tau, \cdot)\right\|_{L^{\infty}\left(\left\{D_{\tau} \geq R\right\}\right)}, \quad \ell=1,2 .
$$

Here, we use the fact that $D_{t}$ is 1-Lipschitz, hence $D_{t}(x) \leq D_{t}\left(x-t v_{1}\right)+D_{t}\left(t v_{1}\right)=D_{t}\left(x-t v_{1}\right)$, and that $D_{\tau}\left(x-(t-\tau) v_{1}\right) \geq D_{t}(x)$ since, $\Gamma$ being convex,

$$
\begin{aligned}
D_{\tau}\left(x-(t-\tau) v_{1}\right) & =\inf \left\{\left|x-(t-\tau) v_{1}-\tau u\right|, \quad u \in \Gamma\right\} \\
& =\inf \left\{\left|x-t\left[(1-\tau / t) v_{1}+(\tau / t) u\right]\right|, \quad u \in \Gamma\right\} \geq \inf \{|x-t U|, \quad U \in \Gamma\}=D_{t}(x) .
\end{aligned}
$$

Therefore, using the decay of $A_{3}$,

$$
D_{t}^{\mu}(x)\left|A_{1}\right|(t, x) \leq a_{1}(0)+\left|\sigma_{1}\right| C_{0}\left(A_{3}\right)\left(\sup _{t^{\prime} \in[0, t]} a_{2}\left(t^{\prime}\right)\right) \int_{0}^{t} \frac{d \tau}{1+\left|x-(t-\tau) v_{1}\right|^{\nu}} .
$$

Similarly, we obtain for $A_{2}$

$$
D_{t}^{\mu}(x)\left|A_{2}\right|(t, x) \leq a_{2}(0)+\left|\sigma_{2}\right| C_{0}\left(A_{3}\right)\left(\sup _{t^{\prime} \in[0, t]} a_{1}\left(t^{\prime}\right)\right) \int_{0}^{t} \frac{d \tau}{1+\left|x-(t-\tau) v_{2}\right|^{\nu}} .
$$

It suffices then to prove that if $R \geq 1$ is large enough, then for $t \geq 0$ and $x \in \Omega$ with $D_{t}(x) \geq R$,

$$
\max _{j=1,2}\left(\left|\sigma_{j}\right| C_{0}\left(A_{3}\right) \int_{0}^{t} \frac{d \tau}{1+\left|x-(t-\tau) v_{j}\right|^{\nu}}\right) \leq \epsilon<1,
$$

with $\epsilon$ independent of $t$ and $x$, since then, one has

$$
\max _{j=1,2} a_{j}(t) \leq \max _{j=1,2} a_{j}(0)+\epsilon\left(\max _{j=1,2} \sup _{t^{\prime} \in[0, t]} a_{j}\left(t^{\prime}\right)\right)
$$

and this will conclude the proof. Letting $s v_{1}, s \in \mathbb{R}$, be the orthogonal projection of $x$ on $\mathbb{R} v_{1}$, we have

$$
\left|x-(t-\tau) v_{1}\right|^{2}=\left|s v_{1}-(t-\tau) v_{1}\right|^{2}+\left|x-s v_{1}\right|^{2} \geq\left|s v_{1}-(t-\tau) v_{1}\right|^{2}+R^{2}
$$


if $s \in[0, t]$ (since then $D_{t}(x)=\left|x-s v_{1}\right| \geq R$ ), and thus

$$
\int_{0}^{t} \frac{d \tau}{1+\left|x-(t-\tau) v_{j}\right|^{\nu}} \leq \int_{0}^{t} \frac{d \tau}{1+\left([s-(t-\tau)]^{2}\left|v_{1}\right|^{2}+R^{2}\right)^{\nu / 2}} \leq \int_{\mathbb{R}} \frac{d \sigma}{1+\left(\sigma^{2}\left|v_{1}\right|^{2}+R^{2}\right)^{\nu / 2}}
$$

which is as small as we want if $R$ is large enough. When $s \geq t$ (the case $s \leq 0$ is identical), then $D_{t}(x)=$ $\left|x-t v_{1}\right| \geq R$ and

$$
\left|x-(t-\tau) v_{1}\right|^{2}=\left|s v_{1}-(t-\tau) v_{1}\right|^{2}+\left|x-s v_{1}\right|^{2}=(s-(t-\tau))^{2}\left|v_{1}\right|^{2}+\left|x-s v_{1}\right|^{2}
$$

so that

$$
\int_{0}^{t} \frac{d \tau}{1+\left|x-(t-\tau) v_{j}\right|^{\nu}} \leq K_{\nu} \int_{-\infty}^{t} \frac{d \sigma}{\left[1+\left|x-s v_{1}\right|+(s-\sigma)\left|v_{1}\right|\right]^{\nu}} \leq \frac{K_{\nu}}{\left|v_{1}\right|} \frac{1}{\left[1+\left|x-s v_{1}\right|+(s-t)\left|v_{1}\right|\right]^{\nu-1}} .
$$

Then, $\left|x-s v_{1}\right|+(s-t)\left|v_{1}\right| \geq\left|x-t v_{1}\right| \geq R$ and the last expression can be made as small as we want if $R$ is chosen sufficiently large. The proof is complete.

\subsection{Proof of Proposition 3}

The proof is rather immediate, and proceeds by induction on $s$. Using (6), we have

$$
\frac{d}{d t} \mathcal{N}(A)=0, \quad \text { where } \quad \mathcal{N}(A) \equiv \frac{1}{2} \int_{\Omega}\left|A_{1}\right|^{2}-\frac{\sigma_{1}}{\sigma_{2}}\left|A_{2}\right|^{2} d x \approx\left\|A=\left(A_{1}, A_{2}\right)\right\|_{L^{2}}^{2},
$$

since $\sigma_{1} \sigma_{2}<0$. We then define an $H^{s}$ functional $\mathcal{N}^{s}, s \in \mathbb{N}$, setting $\left(\mathcal{N}^{0}=\mathcal{N}\right)$

$$
\mathcal{N}^{s}(A) \equiv \sum_{|\alpha| \leq s} \mathcal{N}\left(\partial_{x}^{\alpha} A\right) \approx\|A\|_{H^{s}}^{2}
$$

and assume

$$
\mathcal{N}^{s}(A(t)) \leq C_{s}\left(1+t^{s}\right)^{2}\left\|A^{\text {in }}\right\|_{H^{s}}^{2}
$$

for some $s \geq 0$. Applying $\partial_{x}^{\alpha},|\alpha|=s+1$, to (5), we infer

$$
\left\{\begin{array}{l}
\partial_{t} \partial_{x}^{\alpha} A_{1}-v_{1} \partial_{x} \partial_{x}^{\alpha} A_{1}+\sigma_{1} A_{3} \partial_{x}^{\alpha} A_{2}=-\sigma_{1}\left[\partial_{x}^{\alpha}, A_{3}\right] A_{2} \\
\partial_{t} \partial_{x}^{\alpha} A_{2}-v_{2} \partial_{x} \partial_{x}^{\alpha} A_{2}+\sigma_{2} \bar{A}_{3} \partial_{x}^{\alpha} A_{1}=-\sigma_{2}\left[\partial_{x}^{\alpha}, \bar{A}_{3}\right] A_{1}
\end{array}\right.
$$

By the Leibnitz formula, $\left[\partial_{x}^{\alpha}, A_{3}\right] A_{2}$ is a sum of terms of the type $* \partial_{x}^{\alpha-\beta} A_{2} \partial_{x}^{\beta} A_{3}$ for $0 \neq \beta \leq \alpha$ (where the coefficient $*$ depends only on $\alpha$ and $\beta$ ). Hence, by the induction hypothesis, we deduce

$$
\left\|\left[\partial_{x}^{\alpha}, \bar{A}_{3}\right] A_{1}\right\|_{L^{2}}+\left\|\left[\partial_{x}^{\alpha}, A_{3}\right] A_{2}\right\|_{L^{2}} \leq C\left\|A_{3}\right\|_{W^{s+1, \infty}}\left(1+t^{s}\right)\left\|A^{\mathrm{in}}\right\|_{H^{s}},
$$

where $C$ depends only on $s, \sigma_{1}$ and $\sigma_{2}$. Therefore,

$$
\frac{d}{d t} \mathcal{N}\left(\partial_{x}^{\alpha} A\right) \leq C\left\|A_{3}\right\|_{W^{s+1, \infty}}\left(1+t^{s}\right)\left\|A^{\mathrm{in}}\right\|_{H^{s}} \sqrt{\mathcal{N}\left(\partial_{x}^{\alpha} A\right)}
$$

and the result follows.

\subsection{Proof of Proposition 4}

Let us first consider the case $\Omega=\mathbb{R} /(2 \pi \Lambda)$ with $0<\Lambda_{1}=\Lambda<+\infty$. We define the linear operator $T: D(T) \equiv H^{1}(\Omega) \subset L^{2}(\Omega) \rightarrow L^{2}(\Omega)$ by

$$
T\left(\begin{array}{c}
\psi_{1} \\
\psi_{2}
\end{array}\right) \equiv\left(\begin{array}{c}
i v_{1} \psi_{1}^{\prime}+i \sigma_{1} A_{3} \psi_{2} \\
i v_{2} \psi_{2}^{\prime}+i \sigma_{2} \bar{A}_{3} \psi_{1}
\end{array}\right)
$$

Since $\sigma_{1} \sigma_{2}<0$, we can consider the scalar product, for $\psi=\left(\psi_{1}, \psi_{2}\right)$ and $\phi=\left(\phi_{1}, \phi_{2}\right)$,

$$
\langle\psi, \phi\rangle=\left\langle\left(\begin{array}{c}
\psi_{1} \\
\psi_{2}
\end{array}\right) \mid\left(\begin{array}{c}
\phi_{1} \\
\phi_{2}
\end{array}\right)\right\rangle \equiv \int_{\Omega} \psi_{1} \bar{\phi}_{1}-\frac{\sigma_{1}}{\sigma_{2}} \psi_{2} \bar{\phi}_{2} d x
$$


which is equivalent to the standard scalar product in $L^{2}(\Omega)$ and for which $T$ is self-adjoint. Furthermore, $T$ has compact resolvent. Indeed, for $\mu \in \mathbb{C}$ with $\operatorname{Im}(\mu) \neq 0$ and $|\mu|$ sufficiently large and $f=\left(f_{1}, f_{2}\right) \in L^{2}(\Omega)$, we may write the equation $(T-\mu) \psi=f$ for the unknown $\psi=\left(\psi_{1}, \psi_{2}\right)$ under the form

$$
\hat{\psi}(n)=\left(\hat{\psi}_{1}, \hat{\psi}_{2}\right)(n)=\left(\frac{i \hat{A}_{3} * \hat{\psi}_{2}(n)-\hat{f}_{1}(n)}{\mu+v_{1} n / \Lambda_{1}}, \frac{i \hat{\bar{A}}_{3} * \hat{\psi}_{1}(n)-\hat{f}_{2}(n)}{\mu+v_{2} n / \Lambda_{1}}\right)
$$

where the $(\hat{\psi}(n))_{n \in \mathbb{Z}}$ are the Fourier coefficients of $\psi$. This last equation is easily solved by a contraction argument (for $|\mu|$ large enough) in $\ell^{2}(\mathbb{Z})$, and the easy estimate $\|\psi\|_{H^{1}(\Omega)} \leq C_{\mu}\|f\|_{L^{2}(\Omega)}$ allows to conclude to the compactness of $(T-\mu)^{-1}$ by compact Sobolev embedding. By standard theory, there exists a Hilbert basis (for the scalar product $\langle\cdot \mid \cdot\rangle$, equivalent to the standard scalar product in $L^{2}(\Omega)$ ) of eigenvectors of $T$, denoted $\left(e_{n}\right)_{n \in \mathbb{N}}$, with corresponding real eigenvalues $\left(\lambda_{n}\right)_{n \in \mathbb{N}}$, with $\left|\lambda_{n}\right| \rightarrow+\infty$ as $n \rightarrow+\infty$. As a consequence, if $\psi=\left(\psi_{2}, \psi_{2}\right) \in H^{1}(\Omega)$ and if we denote $c_{n} \equiv\left\langle\psi \mid e_{n}\right\rangle, n \in \mathbb{N}$, we have

$$
\langle T \psi \mid T \psi\rangle=\left\langle\sum_{n \in \mathbb{N}} \lambda_{n} c_{n} e_{n} \mid \sum_{n \in \mathbb{N}} \lambda_{n} c_{n} e_{n}\right\rangle=\sum_{n \in \mathbb{N}} \lambda_{n}^{2}\left|c_{n}\right|^{2} .
$$

Since $A_{3} \in L^{\infty}(\Omega)$, we have

$$
\left\|\left(A_{3} \psi_{1}, \bar{A}_{3} \psi_{2}\right)\right\|_{L^{2}(\Omega)}^{2} \leq C\|\psi\|_{L^{2}(\Omega)}^{2} \leq C\langle\psi \mid \psi\rangle=C \sum_{n \in \mathbb{N}}\left|c_{n}\right|^{2}
$$

and thus, since $v_{1}, v_{2}$ are nonzero by hypothesis, we infer

$\left\|\psi^{\prime}\right\|_{L^{2}(\Omega)}^{2} \approx\left\langle\left(i v_{1} \psi_{1}^{\prime}, i v_{2} \psi_{2}^{\prime}\right) \mid\left(i v_{1} \psi_{1}^{\prime}, i v_{2} \psi_{2}^{\prime}\right)\right\rangle=\langle T \psi \mid T \psi\rangle+\mathcal{O}\left(\left\|\left(A_{3} \psi_{1}, \bar{A}_{3} \psi_{2}\right)\right\|_{L^{2}(\Omega)}^{2}\right)=\sum_{n \in \mathbb{N}} \lambda_{n}^{2}\left|c_{n}\right|^{2}+\mathcal{O}\left(\sum_{n \in \mathbb{N}}\left|c_{n}\right|^{2}\right)$

and consequently, we have the equivalence of (squared) norms

$$
\|\psi\|_{H^{1}(\Omega)}^{2} \approx \sum_{n \in \mathbb{N}}\left(1+\lambda_{n}^{2}\right)\left|c_{n}\right|^{2} .
$$

More generally, by Leibnitz formula (recall that $A_{3} \in W^{s, \infty}(\Omega)$ ), we deduce analogously that for any $s \in \mathbb{N}$, we have the equivalence of (squared) norms

$$
\|\psi\|_{H^{s}(\Omega)}^{2} \approx \sum_{n \in \mathbb{N}}\left(1+\lambda_{n}^{2 s}\right)\left|c_{n}\right|^{2} .
$$

We now turn to the proof of Proposition 4. For any initial data $A^{\text {in }}=\left(A_{1}^{\text {in }}, A_{2}^{\text {in }}\right) \in H^{s}(\Omega)$, we may write

$$
A^{\mathrm{in}}=\sum_{n \in \mathbb{N}}\left\langle A^{\mathrm{in}} \mid e_{n}\right\rangle e_{n}=\sum_{n \in \mathbb{N}} c_{n} e_{n}
$$

in $H^{s}(\Omega)$, and the solution $A=\left(A_{1}, A_{2}\right)$ of $(5)$ is

$$
A(t)=\sum_{n \in \mathbb{N}} \mathrm{e}^{i t \lambda_{n}} c_{n} e_{n}
$$

Hence, we have, using (16),

$$
\|A(t)\|_{H^{s}(\Omega)}^{2} \leq C \sum_{n \in \mathbb{N}}\left(1+\lambda_{n}^{2 s}\right)\left|\mathrm{e}^{i t \lambda_{n}} c_{n}\right|^{2}=C \sum_{n \in \mathbb{N}}\left(1+\lambda_{n}^{2 s}\right)\left|c_{n}\right|^{2} \leq C\left\|A^{\mathrm{in}}\right\|_{H^{s}(\Omega)}^{2}
$$

as required.

We now turn to the case $\Omega=\mathbb{R}$, i.e. $\Lambda=\Lambda_{1}=+\infty$. The point is also to expand on eigenvectors, replacing in the expansion $\psi=\sum_{n=0}^{+\infty} c_{n} e_{n}$ the series by an integral, in the same way we obtain Fourier transform from Fourier series. The idea is naturally to let $\Lambda \rightarrow+\infty$ in the previous case. When $T$ is a second order, self-adjoint operator, this is the well-known Weyl-Stone-Titchmarsh-Kodaira theory (see, e.g. [11], Chap. 5, 
or [5], Chap. 10). The extension to the operator $T$ is not difficult, and we just give a few details for sake of completeness.

For $\lambda \in \mathbb{R}$, let us denote $\left(\Phi^{1}(\cdot, \lambda), \Phi^{2}(\cdot, \lambda)\right)$ the solutions of

$$
T \Phi^{1}-\lambda \Phi^{1}=T \Phi^{2}-\lambda \Phi^{2}=0, \quad \Phi^{1}(x=0, \lambda)=(1,0), \quad \Phi^{2}(x=0, \lambda)=(0,1) .
$$

By classical results, $\Phi^{1}$ and $\Phi^{2}$ are smooth functions of $\lambda$ for fixed $x$. For $0<\Lambda<\infty$, we denote $\left(e_{n}^{\Lambda}\right)_{n \geq 0}$ an Hilbert basis for $T: H^{1}\left(\Omega_{\Lambda}\right) \rightarrow L^{2}\left(\Omega_{\Lambda}\right),\left(\lambda_{n}^{\Lambda}\right)_{n \geq 0}$ the corresponding eigenvalues, and $\langle\cdot \mid \cdot\rangle_{\Lambda}$ and $\langle\cdot \mid \cdot\rangle$ will denote the scalar product in $L^{2}\left(\Omega_{\Lambda}\right)$ and $L^{2}(\mathbb{R})$. We expand the eigenvectors $e_{n}^{\Lambda}, n \in \mathbb{N}$, on the basis of solutions $\left(\Phi^{1}\left(\cdot, \lambda_{n}^{\Lambda}\right), \Phi^{2}\left(\cdot, \lambda_{n}^{\Lambda}\right)\right)$ :

$$
e_{n}^{\Lambda}=\sum_{j=1}^{2} r_{j, n}^{\Lambda} \Phi^{j}\left(\cdot, \lambda_{n}^{\Lambda}\right) \quad r_{j, n}^{\Lambda} \in \mathbb{C} .
$$

If $\psi \in \mathcal{C}_{c}(\mathbb{R})$, we can view $\psi$ as an element of $L^{2}\left(\Omega_{\Lambda}\right)$ provided $\Lambda$ is sufficiently large so that $\operatorname{Supp}(\psi) \subset$ $[-\pi \Lambda,+\pi \Lambda]$, say $\Lambda \geq \Lambda_{0}$. Then, the formula $\psi=\sum_{n=0}^{+\infty} c_{n}^{\Lambda} e_{n}^{\Lambda}$ in $L^{2}\left(\Omega_{\Lambda}\right)$, for $\Lambda \geq \Lambda_{0}$ can be written, in $L^{2}\left(\Omega_{\Lambda}\right)$,

$$
\psi(x)=\sum_{n=0}^{+\infty} c_{n}^{\Lambda} e_{n}^{\Lambda}(x)=\sum_{n=0}^{+\infty} \sum_{j, k=1}^{2}\left\langle\psi \mid \Phi^{k}\left(\cdot, \lambda_{n}^{\Lambda}\right)\right\rangle r_{j, n}^{\Lambda} \bar{r}_{k, n}^{\Lambda} \Phi^{j}\left(x, \lambda_{n}^{\Lambda}\right)=\sum_{j, k=1}^{2} \int_{\mathbb{R}} \tilde{\psi}^{k}(\lambda) \Phi^{j}(x, \lambda) d \rho_{j, k}^{\Lambda}(\lambda),
$$

in the sense of Stieltjes integrals, where (recall that $\psi$ is compactly supported)

$$
\tilde{\psi}^{k}(\lambda) \equiv\left\langle\psi \mid \Phi^{k}(\cdot, \lambda)\right\rangle \quad(k=1,2)
$$

and $\rho_{j, k}^{\Lambda}$ is a piecewise constant function, normalized so that $\rho_{j, k}^{\Lambda}(0+0)=0$, and with discontinuities at the $\lambda_{n}^{\Lambda}$ 's, where

$$
\rho_{j, k}^{\Lambda}\left(\lambda_{n}^{\Lambda}+0\right)-\rho_{j, k}^{\Lambda}\left(\lambda_{n}^{\Lambda}-0\right)=\sum_{m, \lambda_{m}^{\Lambda}=\lambda_{n}^{\Lambda}} r_{j, m}^{\Lambda} \bar{r}_{k, m}^{\Lambda} .
$$

We observe that the right-hand side is a nonnegative hermitian matrix. The Parseval identity for $\psi$ becomes

$$
\langle\psi \mid \psi\rangle=\langle\psi \mid \psi\rangle_{\Lambda}=\sum_{j, k=1}^{2} \int_{\mathbb{R}} \tilde{\psi}^{k}(\lambda) \overline{\tilde{\psi}^{j}(\lambda)} d \rho_{j, k}^{\Lambda}(\lambda)
$$

In order to prove local in space compactness for the matrix $\rho^{\Lambda}=\left(\rho_{j, k}^{\Lambda}\right)_{1 \leq j, k \leq 2}$, we shall prove that for any $\Lambda_{0}>0$, there exists some $\eta>0$ and a constant $C\left(\Lambda_{0}\right)$ such that, if $\left|\lambda_{0}\right| \leq \Lambda_{0} \leq \Lambda-1$, then

$$
\sum_{j, k=1}^{2} \int_{\lambda_{0}-\eta}^{\lambda_{0}+\eta}\left|d \rho_{j, k}^{\Lambda}\right|(\lambda) \leq C\left(\Lambda_{0}\right)
$$

First, from the definition of $\rho^{\Lambda}$, we have

$$
2 \int_{\lambda_{0}-\eta}^{\lambda_{0}+\eta}\left|d \rho_{1,2}^{\Lambda}\right|(\lambda) \leq \int_{\lambda_{0}-\eta}^{\lambda_{0}+\eta} d \rho_{1,1}^{\Lambda}(\lambda)+\int_{\lambda_{0}-\eta}^{\lambda_{0}+\eta} d \rho_{2,2}^{\Lambda}(\lambda),
$$

hence, for (19), it suffices to show that

$$
\int_{\lambda_{0}-\eta}^{\lambda_{0}+\eta} d \rho_{1,1}^{\Lambda}(\lambda)+\int_{\lambda_{0}-\eta}^{\lambda_{0}+\eta} d \rho_{2,2}^{\Lambda}(\lambda) \leq C\left(\Lambda_{0}\right)
$$

For fixed $\Lambda_{0}>0, \lambda_{0} \in\left[-\Lambda_{0},+\Lambda_{0}\right]$ and $\Lambda \geq \Lambda_{0}+1$, we choose $\chi \in \mathcal{C}_{c}((-1,+1))$ with $\int \chi=1$ and for $\psi$ the mollifier $\psi_{\epsilon}(x)=\epsilon^{-1} \chi(x / \epsilon)\left(1,-\sigma_{2} / \sigma_{1}\right)$. Then, we infer, by continuity of the $\Phi^{k}, k=1,2$ that, as $\epsilon \rightarrow 0$,

$$
\tilde{\psi}_{\epsilon}^{k}(\lambda) \rightarrow\left\langle(1,1) \mid \Phi^{k}(0, \lambda)\right\rangle=1
$$

for $k=1$ and $k=2$ locally uniformly in $\lambda$. As a consequence, there exists $\eta>0$, independent of $\Lambda$, such that, for $\epsilon$ small enough,

$$
\sum_{j, k=1}^{2} \int_{\mathbb{R}} \tilde{\psi}_{\epsilon}^{k}(\lambda) \overline{\tilde{\psi}_{\epsilon}^{j}(\lambda)} d \rho_{j, k}^{\Lambda}(\lambda) \geq \frac{1}{2} \int_{\lambda_{0}-\eta}^{\lambda_{0}+\eta} d \rho_{1,1}^{\Lambda}(\lambda)+\frac{1}{2} \int_{\lambda_{0}-\eta}^{\lambda_{0}+\eta} d \rho_{2,2}^{\Lambda}(\lambda)-\frac{1}{4} \int_{\lambda_{0}-\eta}^{\lambda_{0}+\eta}\left|d \rho_{1,2}^{\Lambda}\right|(\lambda) .
$$


Inserting (20) and the Parseval identity, we obtain, for $\epsilon$ sufficiently small, $\Lambda \geq \Lambda_{0}+1$ and $\left|\lambda_{0}\right| \leq \Lambda_{0}$,

$$
\left\langle\psi_{\epsilon} \mid \psi_{\epsilon}\right\rangle \geq \frac{1}{4} \int_{\lambda_{0}-\eta}^{\lambda_{0}+\eta} d \rho_{1,1}^{\Lambda}(\lambda)+\frac{1}{4} \int_{\lambda_{0}-\eta}^{\lambda_{0}+\eta} d \rho_{2,2}^{\Lambda}(\lambda)
$$

which yields (21), and thus (19).

Therefore, on every bounded interval $\left[-\Lambda_{0},+\Lambda_{0}\right]$, the functions $\left(\rho_{j, k}^{\Lambda}\right)_{1 \leq j, k \leq 2}$ have bounded variation, with a uniform bound for $\Lambda \geq \Lambda_{0}+1$. By Helly's compactness theorem, there exists a matrix valued mapping $\rho$ and a sequence $\Lambda_{q} \rightarrow+\infty$ such that $\left(\rho_{j, k}^{\Lambda_{q}}\right)_{1 \leq j, k \leq 2}$ converges to $\rho$ pointwise and weakly in $B V(I)$ for every bounded interval $I$ as $q \rightarrow=\infty$. This allows to pass to the limit in (17):

$$
\psi(x)=\sum_{j, k=1}^{2} \int_{\mathbb{R}} \tilde{\psi}^{k}(\lambda) \Phi^{j}(x, \lambda) d \rho_{j, k}(\lambda) \quad \text { in } L^{2}(\mathbb{R})
$$

and in (18)

$$
\langle\psi \mid \psi\rangle=\sum_{j, k=1}^{2} \int_{\mathbb{R}} \tilde{\psi}^{k}(\lambda) \overline{\tilde{\psi}^{j}(\lambda)} d \rho_{j, k}(\lambda) .
$$

These formulas are extended by density if $\psi \in L^{2}(\mathbb{R})$. Finally, we have, for $\psi \in H^{1}(\mathbb{R})$,

$$
T \psi(x)=\sum_{j, k=1}^{2} \int_{\mathbb{R}} \lambda \tilde{\psi}^{k}(\lambda) \Phi^{j}(x, \lambda) d \rho_{j, k}(\lambda) \quad \text { in } L^{2}(\mathbb{R})
$$

and, as in the periodic case, since $v_{1}$ and $v_{2}$ are non zero,

$$
\left\|\psi^{\prime}\right\|_{L^{2}(\mathbb{R})}^{2} \approx\left\langle\left( i v_{1} \psi_{1}^{\prime}\left|i v_{2} \psi_{2}^{\prime}\right\rangle=\langle T \psi \mid T \psi\rangle+\mathcal{O}\left(\|\psi\|_{L^{2}(\mathbb{R})}^{2}\right)\right.\right.
$$

since $A_{3} \in L^{\infty}(\mathbb{R})$. Hence,

$$
\|\psi\|_{H^{1}(\mathbb{R})}^{2} \approx \sum_{j, k=1}^{2} \int_{\mathbb{R}}\left(1+\lambda^{2}\right) \tilde{\psi}^{k}(\lambda) \overline{\tilde{\psi}^{j}(\lambda)} d \rho_{j, k}(\lambda),
$$

and, for $s \in \mathbb{N}$ arbitrary,

$$
\|\psi\|_{H^{s}(\mathbb{R})}^{2} \approx \sum_{j, k=1}^{2} \int_{\mathbb{R}}\left(1+\lambda^{2 s}\right) \tilde{\psi}^{k}(\lambda) \overline{\tilde{\psi}^{j}(\lambda)} d \rho_{j, k}(\lambda) .
$$

The conclusion then follows. It can be actually shown (as in [5]) that $\rho$ is unique up to some constant matrix and that $\rho^{\Lambda}$ converges to $\rho$ as $\Lambda \rightarrow+\infty$ (locally in space) and not only for some sequence.

\subsection{Proof of Proposition 5}

From the system (10), we deduce, for $0 \leq t^{\prime} \leq t$,

$$
\begin{aligned}
\left|A_{1}\right|\left(t^{\prime}, x\right) & \leq\left\|A_{1}^{\mathrm{in}}\right\|_{L^{\infty}}+\left|\sigma_{1}\right| \int_{0}^{t^{\prime}}\left\|A_{2}(\tau, \cdot)\right\|_{L^{\infty}}\left|A_{3}\right|\left(x-\left(t^{\prime}-\tau\right) v_{1}\right) d \tau \\
& \leq\left\|A_{1}^{\mathrm{in}}\right\|_{L^{\infty}}+\frac{\left|\sigma_{1}\right|}{\left|v_{1}\right|}\left(\sup _{0 \leq \tau \leq t}\left\|A_{2}(\tau, \cdot)\right\|_{L^{\infty}}\right)\left(\sup _{x \in \Omega} \int_{x+\mathbb{R} v_{1}}\left|A_{3}\right|\right)
\end{aligned}
$$

and similarly

$$
\left|A_{2}\right|\left(t^{\prime}, x\right) \leq\left\|A_{2}^{\mathrm{in}}\right\|_{L^{\infty}}+\frac{\left|\sigma_{2}\right|}{\left|v_{2}\right|}\left(\sup _{0 \leq \tau \leq t}\left\|A_{1}(\tau, \cdot)\right\|_{L^{\infty}}\right)\left(\sup _{x \in \Omega} \int_{x+\mathbb{R} v_{2}}\left|A_{3}\right|\right) .
$$

As a consequence,

$$
\left\|A_{1}\left(t^{\prime}, \cdot\right)\right\|_{L^{\infty}} \leq\left\|A_{1}^{\mathrm{in}}\right\|_{L^{\infty}}+\frac{\left|\sigma_{1}\right|}{\left|v_{1}\right|}\left\|A_{2}^{\mathrm{in}}\right\|_{L^{\infty}}\left(\sup _{x \in \Omega} \int_{x+\mathbb{R} v_{1}}\left|A_{3}\right|\right)+\delta \sup _{0 \leq \tau \leq t}\left\|A_{1}(\tau, \cdot)\right\|_{L^{\infty}}
$$


with

$$
\delta \equiv \frac{\left|\sigma_{1} \sigma_{2}\right|}{\left|v_{1}\right| \cdot\left|v_{2}\right|}\left(\sup _{x \in \Omega} \int_{x+\mathbb{R} v_{1}}\left|A_{3}\right|\right)\left(\sup _{x \in \Omega} \int_{x+\mathbb{R} v_{2}}\left|A_{3}\right|\right) .
$$

From the smallness assumption $(11), \delta<1$ and it follows

$$
\sup _{0 \leq t^{\prime} \leq t}\left\|A_{1}(\tau, \cdot)\right\|_{L^{\infty}} \leq \frac{1}{1-\delta}\left(\left\|A_{1}^{\text {in }}\right\|_{L^{\infty}}+\frac{\left|\sigma_{1}\right|}{\left|v_{1}\right|}\left\|A_{2}^{\text {in }}\right\|_{L^{\infty}} \sup _{x \in \Omega} \int_{x+\mathbb{R} v_{1}}\left|A_{3}\right|\right)
$$

and analogously for $A_{2}$. Therefore, the estimate for $s=0$ is proved: there exists $C>0$ such that, for $t \geq 0$,

$$
\left\|\Sigma_{t} A^{\mathrm{in}}\right\|_{L^{\infty}} \leq C\left\|A^{\mathrm{in}}\right\|_{L^{\infty}} .
$$

We now argue by induction and assume the result for $s \in \mathbb{N}$. Let $\alpha \in \mathbb{N}^{d}$ with $|\alpha|=s+1$, and let us apply $\partial_{x}^{\alpha}$ to (10) to obtain

$$
\left\{\begin{aligned}
\partial_{x}^{\alpha} A_{1}(t, x)=\partial_{x}^{\alpha} A_{1}^{\text {in }}\left(x-t v_{1}\right) & -\sigma_{1} \int_{0}^{t} \partial_{x}^{\alpha} A_{2}\left(\tau, x-(t-\tau) v_{1}\right) A_{3}\left(x-(t-\tau) v_{1}\right) d \tau \\
& -\sigma_{1} \int_{0}^{t}\left[\partial_{x}^{\alpha}, A_{3}\left(x-(t-\tau) v_{1}\right)\right] A_{2}\left(\tau, x-(t-\tau) v_{1}\right) d \tau \\
& -\sigma_{2} \int_{0}^{t} \partial_{x}^{\alpha} A_{1}\left(\tau, x-(t-\tau) v_{2}\right) \bar{A}_{3}\left(x-(t-\tau) v_{2}\right) d \tau \\
\partial_{x}^{\alpha} A_{2}(t, x)=\partial_{x}^{\alpha} A_{2}^{\text {in }}\left(x-t v_{2}\right) & -\sigma_{2} \int_{0}^{t}\left[\partial_{x}^{\alpha}, \bar{A}_{3}\left(x-(t-\tau) v_{2}\right)\right] A_{1}\left(\tau, x-(t-\tau) v_{2}\right) d \tau .
\end{aligned}\right.
$$

Using the induction hypothesis and the Leibnitz formula, we infer, for $t \geq 0$,

$$
\begin{aligned}
\mid \int_{0}^{t}\left[\partial_{x}^{\alpha}, A_{3}\left(x-(t-\tau) v_{1}\right)\right] A_{2}(\tau, x-( & \left.t-\tau) v_{1}\right) d \tau \mid \\
& \leq C_{\alpha}\left\|A_{2}\right\|_{L^{\infty}\left(\mathbb{R}_{+}, W^{s, \infty}\right)} \max _{\beta \leq \alpha} \int_{0}^{t}\left|\partial_{x}^{\beta} A_{3}\left(x-(t-\tau) v_{1}\right)\right| d \tau \\
& \leq C_{s}\left\|A^{\text {in }}\right\|_{W^{s, \infty}},
\end{aligned}
$$

and similarly for the last integral. Then, arguing as for the case $s=0$, we infer for $0 \leq t^{\prime} \leq t$

$$
\left\|\partial_{x}^{\alpha} A_{1}\left(t^{\prime}, \cdot\right)\right\|_{L^{\infty}} \leq C_{s}\left\|A^{\mathrm{in}}\right\|_{W^{s+1, \infty}}+\delta \sup _{0 \leq \tau \leq t}\left\|\partial_{x}^{\alpha} A_{1}(\tau, \cdot)\right\|_{L^{\infty}}
$$

and similarly for $A_{2}$, which allows to conclude.

\subsection{Proof of Proposition 6}

We recall that, here, $\sigma_{1} \sigma_{2}>0$ and $A_{3} \not \equiv 0$, hence $\gamma>0$. To prove the growth estimate for $\Sigma_{t}$, we argue as for Proposition 5 by induction on $s$. The first point is to find a suitable norm. For the ODE part (in time) of (5), namely

$$
\frac{d A}{d t}+\left(\begin{array}{cc}
0 & \sigma_{1} A_{3} \\
\sigma_{2} \bar{A}_{3} & 0
\end{array}\right) A=0
$$

in which the matrix has eigenvalues $\pm \sqrt{\sigma_{1} \sigma_{2}}\left|A_{3}\right|$, it turns out that the norm

$$
\nu_{0}(A) \equiv\left|A_{1}\right|+\sqrt{\frac{\sigma_{1}}{\sigma_{2}}}\left|A_{2}\right|
$$

is well adapted, since

$$
\nu_{0}\left(\left(\begin{array}{cc}
0 & \sigma_{1} A_{3} \\
\sigma_{2} \bar{A}_{3} & 0
\end{array}\right) A\right)=\sqrt{\sigma_{1} \sigma_{2}}\left|A_{3}\right| \nu_{0}(A) .
$$

Therefore, for $1 \leq p \leq \infty$, it natural to work with the norm in $L^{p}\left(\Omega, \mathbb{C}^{2}\right)$

$$
\mathscr{N}_{p}(A) \equiv\left\|A_{1}\right\|_{L^{p}}+\sqrt{\frac{\sigma_{1}}{\sigma_{2}}}\left\|A_{2}\right\|_{L^{p}}
$$


Taking the $L^{p}$ norm in (10) yields

$$
\left\{\begin{array}{l}
\left\|A_{1}(t)\right\|_{L^{p}} \leq\left\|A_{1}^{\mathrm{in}}\right\|_{L^{p}}+\left|\sigma_{1}\right| \cdot\left\|A_{3}\right\|_{L^{\infty}} \int_{0}^{t}\left\|A_{2}(\tau)\right\|_{L^{p}} d \tau \\
\left\|A_{2}(t)\right\|_{L^{p}} \leq\left\|A_{2}^{\mathrm{in}}\right\|_{L^{p}}+\left|\sigma_{2}\right| \cdot\left\|A_{3}\right\|_{L^{\infty}} \int_{0}^{t}\left\|A_{1}(\tau)\right\|_{L^{p}} d \tau
\end{array}\right.
$$

hence

$$
\mathscr{N}_{p}(A(t)) \leq \mathscr{N}_{p}\left(A^{\mathrm{in}}\right)+\sqrt{\sigma_{1} \sigma_{2}}\left\|A_{3}\right\|_{L^{\infty}} \int_{0}^{t} \mathscr{N}_{p}(A(\tau)) d \tau
$$

and the Gronwall lemma gives as wished, for $t \geq 0$,

$$
\mathscr{N}_{p}(A(t)) \leq \mathscr{N}_{p}\left(A^{\mathrm{in}}\right) \mathrm{e}^{\gamma t}
$$

We assume now that, for some $s \in \mathbb{N}$, we have for $t \geq 0$

$$
\|A(t)\|_{W^{s, p}} \leq K_{s}\left(A_{3}\right)\left\|A^{\text {in }}\right\|_{W^{s, p}}\left(1+t^{s}\right) \mathrm{e}^{\gamma t} .
$$

We apply $\partial_{x}^{\alpha},|\alpha|=s+1$, to (10):

$$
\left\{\begin{aligned}
\partial_{x}^{\alpha} A_{1}(t, x)=\partial_{x}^{\alpha} A_{1}^{\mathrm{in}}\left(x-t v_{1}\right) & -\sigma_{1} \int_{0}^{t} \partial_{x}^{\alpha} A_{2}\left(\tau, x-(t-\tau) v_{1}\right) A_{3}\left(x-(t-\tau) v_{1}\right) d \tau \\
& -\sigma_{1} \int_{0}^{t}\left[\partial_{x}^{\alpha}, A_{3}\left(x-(t-\tau) v_{1}\right)\right] A_{2}\left(\tau, x-(t-\tau) v_{1}\right) d \tau \\
\partial_{x}^{\alpha} A_{2}(t, x)=\partial_{x}^{\alpha} A_{2}^{\mathrm{in}}\left(x-t v_{2}\right) & -\sigma_{2} \int_{0}^{t} \partial_{x}^{\alpha} A_{1}\left(\tau, x-(t-\tau) v_{2}\right) \bar{A}_{3}\left(x-(t-\tau) v_{2}\right) d \tau \\
& -\sigma_{2} \int_{0}^{t}\left[\partial_{x}^{\alpha}, \bar{A}_{3}\left(x-(t-\tau) v_{2}\right)\right] A_{1}\left(\tau, x-(t-\tau) v_{2}\right) d \tau
\end{aligned}\right.
$$

Using the commutator estimate

$$
\left\|\int_{0}^{t}\left[\partial_{x}^{\alpha}, A_{3}\left(x-(t-\tau) v_{1}\right)\right] A_{2}\left(\tau, x-(t-\tau) v_{1}\right) d \tau\right\|_{L^{p}} \leq C_{\alpha}\left\|A_{3}\right\|_{W^{s+1, \infty}} \int_{0}^{t}\left\|A_{2}(\tau)\right\|_{W^{s, p}} d \tau
$$

and the fact that, by the induction hypothesis,

$$
\int_{0}^{t}\left\|A_{2}(\tau)\right\|_{W^{s, p}} d \tau \leq K_{s}\left(A_{3}\right)\left\|A^{\mathrm{in}}\right\|_{W^{s, p}} \int_{0}^{t}\left(1+\tau^{s}\right) \mathrm{e}^{\gamma \tau} d \tau \leq K\left\|A^{\mathrm{in}}\right\|_{W^{s, p}}\left(1+t^{s}\right) \mathrm{e}^{\gamma t}
$$

since $\gamma>0$ (and similarly for the other term), we have

$$
\left\{\begin{array}{l}
\left\|\partial_{x}^{\alpha} A_{1}(t)\right\|_{L^{p}} \leq\left\|\partial_{x}^{\alpha} A_{1}^{\mathrm{in}}\right\|_{L^{p}}+\left|\sigma_{1}\right| \cdot\left\|A_{3}\right\|_{L^{\infty}} \int_{0}^{t}\left\|\partial_{x}^{\alpha} A_{2}(\tau)\right\|_{L^{p}} d \tau+K\left\|A^{\mathrm{in}}\right\|_{W^{s, p}}\left(1+t^{s}\right) \mathrm{e}^{\gamma t} \\
\left\|\partial_{x}^{\alpha} A_{2}(t)\right\|_{L^{p}} \leq\left\|\partial_{x}^{\alpha} A_{2}^{\mathrm{in}}\right\|_{L^{p}}+\left|\sigma_{2}\right| \cdot\left\|A_{3}\right\|_{L^{\infty}} \int_{0}^{t}\left\|\partial_{x}^{\alpha} A_{1}(\tau)\right\|_{L^{p}} d \tau+K\left\|A^{\mathrm{in}}\right\|_{W^{s, p}}\left(1+t^{s}\right) \mathrm{e}^{\gamma t}
\end{array}\right.
$$

As a consequence,

$$
\begin{aligned}
\mathscr{N}_{p}\left(\partial_{x}^{\alpha} A(t)\right) & \leq \mathscr{N}_{p}\left(\partial_{x}^{\alpha} A^{\mathrm{in}}\right)+K\left\|A^{\mathrm{in}}\right\|_{W^{s, p}}\left(1+t^{s}\right) \mathrm{e}^{\gamma t}+\sqrt{\sigma_{1} \sigma_{2}}\left\|A_{3}\right\|_{L^{\infty}} \int_{0}^{t} \mathscr{N}_{p}\left(\partial_{x}^{\alpha} A(\tau)\right) d \tau \\
& \leq K\left\|A^{\mathrm{in}}\right\|_{W^{s+1, p}}\left(1+t^{s}\right) \mathrm{e}^{\gamma t}+\sqrt{\sigma_{1} \sigma_{2}}\left\|A_{3}\right\|_{L^{\infty}} \int_{0}^{t} \mathscr{N}_{p}\left(\partial_{x}^{\alpha} A(\tau)\right) d \tau
\end{aligned}
$$

and here again, the Gronwall lemma implies, for $t \geq 0$,

$$
\mathscr{N}_{p}\left(\partial_{x}^{\alpha} A(t)\right) \leq K\left\|A^{\mathrm{in}}\right\|_{W^{s+1, p}}\left(1+t^{s}\right) \mathrm{e}^{\gamma t}+\gamma K\left\|A^{\mathrm{in}}\right\|_{W^{s+1, p}} \mathrm{e}^{\gamma t} \int_{0}^{t}\left(1+\tau^{s}\right) d \tau \leq K\left\|A^{\mathrm{in}}\right\|_{W^{s+1, p}}\left(1+t^{s+1}\right) \mathrm{e}^{\gamma t}
$$

as claimed. 


\subsection{Proof of Proposition 7}

The proof of $(i)$ follows by considering functions $\left(A_{1}, A_{2}\right)$ depending only on time. Hence $(5)$ reduces to (9), with eigenvalues $\pm \sqrt{\sigma_{1} \sigma_{2}}\left|A_{3}\right|$ and $(i)$ is proved.

For $(i i)$, we consider functions depending only on the variables $x^{\prime}$ orthogonal to $\operatorname{Span}\left(v_{1}, v_{2}\right)$ (we have $\left.\operatorname{dim} x^{\prime} \geq 1\right)$. Then, (5) reduces to (9) once again, and therefore

$$
A\left(t, x^{\prime}\right)=\exp \left(-t\left(\begin{array}{cc}
0 & \sigma_{1} A_{3}\left(x^{\prime}\right) \\
\sigma_{2} \bar{A}_{3}\left(x^{\prime}\right) & 0
\end{array}\right)\right) A^{\text {in }}\left(x^{\prime}\right) .
$$

If $\varepsilon \in(0, \gamma)$ is given, there exists some $y_{\varepsilon}^{\prime} \in \Omega$ such that $\sqrt{\sigma_{1} \sigma_{2}}\left|A_{3}\right|\left(y_{\varepsilon}^{\prime}\right) \geq \gamma-\varepsilon / 2$, and then, by continuity of $A_{3}, r_{\varepsilon}>0$ such that $\sqrt{\sigma_{1} \sigma_{2}}\left|A_{3}\right|\left(x^{\prime}\right) \geq \gamma-\varepsilon>0$ if $x^{\prime} \in B_{2 r_{\varepsilon}}\left(y_{\varepsilon}^{\prime}\right)$. We then consider the eigenvector (for fixed $\left.x^{\prime}\right)$ for the eigenvalue $\sqrt{\sigma_{1} \sigma_{2}}\left|A_{3}\right|\left(x^{\prime}\right)$

$$
A^{\text {in }}\left(x^{\prime}\right) \equiv c \chi\left(x^{\prime}\right)\left(\begin{array}{c}
1 \\
\operatorname{sgn}\left(\sigma_{1}\right) \sqrt{\frac{\sigma_{2}}{\sigma_{1}}} \frac{\bar{A}_{3}\left(x^{\prime}\right)}{\left|A_{3}\right|\left(x^{\prime}\right)}
\end{array}\right) \in \mathcal{C}_{c}^{\infty}\left(B_{2 r_{\varepsilon}}\left(y_{\varepsilon}^{\prime}\right)\right),
$$

where $\chi \in \mathcal{C}_{c}^{\infty}\left(B_{2 r_{\varepsilon}}\left(y_{\varepsilon}^{\prime}\right),[0,1]\right)$ is equal to one in the ball $B_{r_{\varepsilon}}\left(y_{\varepsilon}^{\prime}\right)$ (in the variables $x^{\prime}$ ), and

$$
c \equiv \frac{1}{\max \left(1, \sqrt{\sigma_{2} / \sigma_{1}}\right)}>0
$$

is just a normalization constant. Hence,

$$
\left|A^{\mathrm{in}}\right|_{\infty}\left(x^{\prime}\right) \leq 1 \quad \text { in } \quad B_{2 r_{\varepsilon}}\left(y_{\varepsilon}^{\prime}\right) \quad \text { and } \quad\left|A^{\mathrm{in}}\right|_{\infty}\left(x^{\prime}\right)=1 \quad \text { in } \quad B_{r_{\varepsilon}}\left(y_{\varepsilon}^{\prime}\right)
$$

and since we have an eigenvector,

$$
A\left(t, x^{\prime}\right)=\mathrm{e}^{t \sqrt{\sigma_{1} \sigma_{2}}\left|A_{3}\right|\left(x^{\prime}\right)} A^{\text {in }}\left(x^{\prime}\right)
$$

and the conclusion follows.

The case (iii) follows the same lines, since the hypothesis allows us to take $\varepsilon=0$ and $r_{\varepsilon}=r>0$. The multiplicity is infinite since the choice of $\chi \in \mathcal{C}_{c}^{\infty}\left(B_{2 r}\left(y^{\prime}\right)\right)$ such that $\chi=1$ on $B_{r}\left(y^{\prime}\right)$ is arbitrary.

The proof of $(i v)$ relies on an explicit computation. Let $v \equiv v_{1}=v_{2}$ and let us recall that $A_{3}$ is assumed real-valued. Then, (5) becomes, in $\Omega=\mathbb{R} /(2 \pi \Lambda \mathbb{Z}$ ) (with $0<\Lambda<\infty$ ),

$$
\left\{\begin{array}{l}
\partial_{t} \tilde{A}_{1}+\sigma_{1} A_{3}(x+t v) \tilde{A}_{2}=0 \\
\partial_{t} \tilde{A}_{2}+\sigma_{2} A_{3}(x+t v) \tilde{A}_{1}=0,
\end{array} \quad \text { where } \quad \tilde{A}_{j}(t, x) \equiv A_{j}(t, x+t v) \quad(j=1,2) .\right.
$$

For fixed $x \in \Omega$, we can solve this ODE explicitly using the fact that the nonsingular matrix

$$
P \equiv\left(\begin{array}{cc}
\sqrt{\sigma_{1} \sigma_{2}} & -\sqrt{\sigma_{1} \sigma_{2}} \\
\sigma_{2} & \sigma_{2}
\end{array}\right)
$$

is independent of $t$ and diagonalizes the system: the vector $B \equiv P^{-1}\left(\tilde{A}_{1}, \tilde{A}_{2}\right)^{t}$ solves

$$
\partial_{t} B+\left(\begin{array}{cc}
\sqrt{\sigma_{1} \sigma_{2}} A_{3}(x+t v) & 0 \\
0 & -\sqrt{\sigma_{1} \sigma_{2}} A_{3}(x+t v)
\end{array}\right) B=0
$$

from which we infer after straightforward computations

$$
\left\{\begin{aligned}
A_{1}(t, x)= & A_{1}^{\mathrm{in}}(x-t v) \cosh \left(\sqrt{\sigma_{1} \sigma_{2}} \int_{0}^{t} A_{3}(x-(t-\tau) v) d \tau\right) \\
& -\operatorname{sgn}\left(\sigma_{1}\right) \sqrt{\frac{\sigma_{1}}{\sigma_{2}}} A_{2}^{\mathrm{in}}(x-t v) \sinh \left(\sqrt{\sigma_{1} \sigma_{2}} \int_{0}^{t} A_{3}(x-(t-\tau) v) d \tau\right) \\
A_{2}(t, x)= & A_{2}^{\mathrm{in}}(x-t v) \cosh \left(\sqrt{\sigma_{1} \sigma_{2}} \int_{0}^{t} A_{3}(x-(t-\tau) v) d \tau\right) \\
& -\operatorname{sgn}\left(\sigma_{1}\right) \sqrt{\frac{\sigma_{2}}{\sigma_{1}}} A_{1}^{\mathrm{in}}(x-t v) \sinh \left(\sqrt{\sigma_{1} \sigma_{2}} \int_{0}^{t} A_{3}(x-(t-\tau) v) d \tau\right) .
\end{aligned}\right.
$$


The first statement in $(i v)$ follows easily from this explicit formula and the fact that

$$
\left|\int_{0}^{t} A_{3}(x-(t-\tau) v) d \tau\right| \leq t\left|\frac{1}{|\Omega|} \int_{\Omega} A_{3}(y) d y\right|+\mathcal{O}(1)
$$

as $t \rightarrow+\infty$ uniformly in $x$. We solve the eigenvalue problem (7) in the same way. For $\lambda=-i \mu \in \mathbb{C}$ with $\operatorname{Im} \lambda=-\operatorname{Re} \mu<0,(7)$ is

$$
\left\{\begin{array}{l}
v \psi_{1}^{\prime}+\sigma_{1} \psi_{2} A_{3}+\mu \psi_{1}=0 \\
v \psi_{2}^{\prime}+\sigma_{2} \psi_{1} A_{3}+\mu \psi_{2}=0
\end{array}\right.
$$

Here again, the vector $\Psi \equiv \mathrm{e}^{\mu x / v} P^{-1} \psi$ solves

$$
v \Psi^{\prime}+\left(\begin{array}{cc}
\sqrt{\sigma_{1} \sigma_{2}} A_{3}(x) & 0 \\
0 & -\sqrt{\sigma_{1} \sigma_{2}} A_{3}(x)
\end{array}\right) \Psi=0,
$$

which yields, for some vector $\zeta=\left(\zeta_{1}, \zeta_{2}\right) \in \mathbb{C}^{2}$,

$$
\left\{\begin{array}{l}
\mathrm{e}^{-\mu x / v} \psi_{1}(t, x)=\zeta_{1} \cosh \left(\frac{\sqrt{\sigma_{1} \sigma_{2}}}{v} \int_{0}^{x} A_{3}(y) d y\right)-\zeta_{2} \operatorname{sgn}\left(\sigma_{1}\right) \sqrt{\frac{\sigma_{1}}{\sigma_{2}}} \sinh \left(\frac{\sqrt{\sigma_{1} \sigma_{2}}}{v} \int_{0}^{x} A_{3}(y) d y\right) \\
\mathrm{e}^{-\mu x / v} \psi_{2}(t, x)=\zeta_{2} \cosh \left(\frac{\sqrt{\sigma_{1} \sigma_{2}}}{v} \int_{0}^{x} A_{3}(y) d y\right)-\zeta_{1} \operatorname{sgn}\left(\sigma_{1}\right) \sqrt{\frac{\sigma_{2}}{\sigma_{1}}} \sinh \left(\frac{\sqrt{\sigma_{1} \sigma_{2}}}{v} \int_{0}^{x} A_{3}(y) d y\right) .
\end{array}\right.
$$

Now, the periodic boundary condition implies that $\mu$ is an eigenvalue for (7) if and only if $\zeta$ is a non trivial solution of (we recall that $2 \pi \Lambda=|\Omega|$ )

$$
\mathrm{e}^{-\mu|\Omega| / v} \zeta=\left(\begin{array}{cc}
\cosh \left(\frac{\sqrt{\sigma_{1} \sigma_{2}}}{v} \int_{0}^{|\Omega|} A_{3}(y) d y\right) & -\operatorname{sgn}\left(\sigma_{1}\right) \sqrt{\frac{\sigma_{1}}{\sigma_{2}}} \sinh \left(\frac{\sqrt{\sigma_{1} \sigma_{2}}}{v} \int_{0}^{|\Omega|} A_{3}(y) d y\right) \\
-\operatorname{sgn}\left(\sigma_{1}\right) \sqrt{\frac{\sigma_{2}}{\sigma_{1}}} \sinh \left(\frac{\sqrt{\sigma_{1} \sigma_{2}}}{v} \int_{0}^{|\Omega|} A_{3}(y) d y\right) & \cosh \left(\frac{\sqrt{\sigma_{1} \sigma_{2}}}{v} \int_{0}^{|\Omega|} A_{3}(y) d y\right)
\end{array}\right) \zeta
$$

Since the matrix on the right-hand side has eigenvalues $\exp \left( \pm \frac{\sqrt{\sigma_{1} \sigma_{2}}}{v} \int_{0}^{|\Omega|} A_{3}(y) d y\right)$, this happens only if, for some $m \in \mathbb{Z}$,

$$
\mu= \pm \frac{\sqrt{\sigma_{1} \sigma_{2}}}{|\Omega|}\left|\int_{0}^{|\Omega|} A_{3}(y) d y\right|+i \frac{2 \pi m v}{|\Omega|}= \pm \sqrt{\sigma_{1} \sigma_{2}}\left|\frac{1}{|\Omega|} \int_{\Omega} A_{3}(y) d y\right|+\frac{i m v}{\Lambda} .
$$

\subsection{Proof of Proposition 8}

We recall that here, $\Omega=\mathbb{R}, \sigma_{1} \sigma_{2}>0$ and $v_{1} v_{2}<0$. Possibly changing the sign of $x$, we may assume, without loss of generality, that $v_{1}>0>v_{2}$.

\subsubsection{Proof of (i)}

We shall first focus on statement $(i)$, where we choose $A_{3}$ to be a multiple of the characteristic function of an interval: $A_{3} \equiv \Gamma \mathbf{1}_{x \in[a, b]}$. We could have assumed $\Gamma \in \mathbb{R}_{+}$by just multiplying $A_{2}$ by a constant phase factor. We look for eigenvalues for $(7)$ with $\lambda=-i \mu, \operatorname{Re}(\mu)>0$, and $\left(\psi_{1}, \psi_{2}\right)$ a non-trivial weak solution of (7), i.e.

$$
\left\{\begin{array}{l}
v_{1} \psi_{1}^{\prime}+\sigma_{1} \psi_{2} A_{3}+\mu \psi_{1}=0 \\
v_{2} \psi_{2}^{\prime}+\sigma_{2} \psi_{1} \bar{A}_{3}+\mu \psi_{2}=0
\end{array}\right.
$$

Since $A_{3}$ vanishes on $(-\infty, a)$ and in view of the fact that $v_{1}>0>v_{2}$, the subspace of bounded solutions of $(24)$ on $(-\infty, b)$ is exactly $\mathbb{C} \psi_{*}$, with

$$
\psi_{*}(x) \equiv \begin{cases}\left(0, \mathrm{e}^{-\mu(x-a) / v_{2}}\right) & \text { on }(-\infty, a] \\ \exp (-(x-a) M)(0,1) & \text { on }[a, b]\end{cases}
$$


and where

$$
M \equiv\left(\begin{array}{cc}
\frac{\mu}{v_{1}} & \frac{\sigma_{1} \mathcal{A}_{3}}{v_{1}} \\
\frac{\sigma_{2} \overline{\mathcal{A}}_{3}}{v_{2}} & \frac{\mu}{v_{2}}
\end{array}\right)
$$

Hence $\mu$ is an eigenvalue for (7) if and only if

$$
[\exp (-(b-a) M)(0,1)]_{2}=0
$$

so that $\psi_{*}$ can be extended to $[b,+\infty)$ by

$$
\psi_{*}(x) \equiv\left(\beta \mathrm{e}^{-\mu(x-b) / v_{1}}, 0\right)
$$

for some nonzero constant $\beta$. Denoting

$$
\gamma \equiv \sqrt{\sigma_{1} \sigma_{2}}\left|\mathcal{A}_{3}\right| \quad \text { and } \quad \hat{\mu} \equiv 2 \gamma \frac{\sqrt{\left|v_{1} v_{2}\right|}}{v_{1}-v_{2}}>0
$$

the matrix $M$ has eigenvalues

$$
\frac{\mu}{2} \frac{v_{1}+v_{2}}{v_{1} v_{2}} \pm \frac{\gamma}{\sqrt{\left|v_{1} v_{2}\right|}} \sqrt{\frac{\mu^{2}}{\hat{\mu}^{2}}-1}=\sigma \pm \gamma \tau
$$

where the last square root stands for one fixed complex-valued square root. Thus, a straightforward computation $^{3}$ gives that

$$
[\exp (-(b-a) M)(0,1)]_{2}=0
$$

if and only if (the fraction has value $b-a$ if $\mu=\hat{\mu}$ )

$$
\exp (-(b-a) \gamma \tau)-\frac{\sinh ((b-a) \gamma \tau)}{\gamma \tau}\left(\frac{\mu}{v_{2}}-\sigma-\gamma \tau\right)=0
$$

or, after some algebra,

$$
\text { for } \mu \neq \hat{\mu}, \quad \exp (2 \gamma(b-a) \tau)=\frac{\frac{\mu}{\hat{\mu}}-\sqrt{\frac{\mu^{2}}{\hat{\mu}^{2}}-1}}{\frac{\mu}{\hat{\mu}}+\sqrt{\frac{\mu^{2}}{\hat{\mu}^{2}}-1}} ; \quad \text { for } \quad \mu=\hat{\mu}, \quad \frac{(b-a) \gamma}{\sqrt{\left|v_{1} v_{2}\right|}}=-1
$$

Therefore, $\mu=\hat{\mu}$ is not an eigenvalue for $(24)$. We assume now $\mu \neq \hat{\mu}$. Since the mapping cosh $: \mathbb{C} \rightarrow \mathbb{C}$ is onto, for $\mu \in \mathbb{C}$, there exists some $y \in \mathbb{C}$ such that

$$
\frac{\mu}{\hat{\mu}}=\cosh (y)
$$

Moreover, since cosh is even and $2 i \pi$-periodic, we can assume $y=u+i v$ with $u \in \mathbb{R}, v \in[0, \pi]$, and since $\mu=\cosh (y)=\cosh (u) \cos (v)+i \sinh (u) \sin (v)$ has positive real part, we have $v \in[0, \pi / 2)$. Then, (25) becomes

$$
\exp (2 \gamma(b-a) \tau)=\frac{\cosh (y)-\sinh (y)}{\cosh (y)+\sinh (y)}=\mathrm{e}^{-2 y}
$$

that is, for some $m \in \mathbb{Z}$,

$$
\gamma(b-a) \tau=\frac{\gamma(b-a)}{\sqrt{\left|v_{1} v_{2}\right|}} \sinh (y)=-y+i m \pi .
$$

\footnotetext{
${ }^{3}$ using that if $A \in \mathcal{M}_{2}(\mathbb{C})$ has two distinct eigenvalues $(\alpha$ and $\beta$ ) or is non diagonalizable (double eigenvalue $\alpha$ ), the formula $\mathrm{e}^{A}=\mathrm{e}^{\alpha}+\frac{\mathrm{e}^{\beta}-\mathrm{e}^{\alpha}}{\beta-\alpha}(A-\alpha)$ holds, where the fraction is $\mathrm{e}^{\alpha}$ if $\beta=\alpha$.
} 
Taking real and imaginary parts, we infer

$$
\alpha \sinh (u) \cos (v)=-u, \quad-v+m \pi=\alpha \cosh (u) \sin (v),
$$

where $\alpha$ is the absolute area:

$$
\alpha \equiv \frac{\sqrt{\sigma_{1} \sigma_{2}}}{\sqrt{\left|v_{1} v_{2}\right|}}\left|\int_{\mathbb{R}} A_{3}(x) d x\right|=\frac{\gamma(b-a)}{\sqrt{\left|v_{1} v_{2}\right|}}>0 .
$$

Since $v \in[0, \pi / 2)$, the first equation in (26) implies $u=0$, so that (26) is now reduced to the single equation

$$
-v+m \pi=\alpha \sin (v),
$$

with $v \in(0, \pi / 2) \quad(v=0$ is excluded since $\mu \neq \hat{\mu})$. Here again, since $v \in(0, \pi / 2)$, it is immediate that (27) has no solution for $m \leq 0$. An elementary graphical analysis shows the following: for $m=1,(27)$ has no solution (in $(0, \pi / 2)$ ) for $\alpha \leq \pi / 2$, and exactly one solution $v \in(0, \pi / 2)$ for $\alpha>\pi / 2$, which goes from $\pi / 2^{-}$(for $\alpha=(\pi / 2)^{+}$) to $0^{+}$(for $\alpha \rightarrow+\infty$ ), that is $\mu$ goes from $0^{+}$to $\hat{\mu}$; for $m=2$, (27) has exactly one solution for $\alpha>3 \pi / 2$, and no solution (in $(0, \pi / 2)$ ) for $\alpha \leq 3 \pi / 2$; for $m \geq 1$, (27) has exactly one solution for $\alpha>(2 m-1) \pi / 2$ (with corresponding $\mu$ going from $0^{+}$to $\hat{\mu}$ ), and no solution for $\alpha \leq(2 m-1) \pi / 2$. When $\alpha$ crosses the values $\alpha=k \pi / 2, k \geq 1$ odd, an eigenvalue 0 crosses the real axis to go to the half-plane $\{\operatorname{Im}<0\}$ up to $-i \hat{\mu}$. Consequently, the equation (27) has no solution for $\alpha \leq \pi / 2$, and (for $m \geq 1$ ) exactly $m$ solutions for $(2 m-1) \pi / 2<\alpha \leq(2 m+1) \pi / 2$.

\subsubsection{Proof of (ii)}

We look for eigenvalues for (7) $\lambda=-i \mu$, with $\mu>0$, and $\psi=\left(\psi_{1}, \psi_{2}\right)$ a non-trivial, real-valued, solution of (7), that is

$$
\left\{\begin{array}{l}
v_{1} \psi_{1}^{\prime}+\sigma_{1} \psi_{2} A_{3}+\mu \psi_{1}=0 \\
v_{2} \psi_{2}^{\prime}+\sigma_{2} \psi_{1} A_{3}+\mu \psi_{2}=0 .
\end{array}\right.
$$

Notice that if $\mu>0$, the behavior of $\psi$ at $\pm \infty$ should be given by the solutions of

$$
v_{1} \psi_{1}^{\prime}+\mu \psi_{1}=v_{2} \psi_{2}^{\prime}+\mu \psi_{2}=0,
$$

which means, since $v_{2}<0<v_{1}$, that one expects the solutions (decaying to zero at infinity) of (28) to be such that, for some real constants $\alpha_{+}, \alpha_{-}$

$$
\psi(x)=\left(\psi_{1}, \psi_{2}\right)(x) \approx \begin{cases}\left(\alpha_{+} \mathrm{e}^{-\mu x / v_{1}}, 0\right) & x \rightarrow+\infty \\ \left(0, \alpha_{-} \mathrm{e}^{-\mu x / v_{2}}\right) & x \rightarrow-\infty .\end{cases}
$$

The first step is to construct particular solutions $\psi_{\mu}^{ \pm}$with the above mentioned asymptotic behavior at $\pm \infty$ up to $\mu=0$. It suffices to treat the case $x \rightarrow-\infty$ (the other case being analogous). We use a classical fixed point argument (see, e.g., [5]), and the point is to have some uniformity for $\mu \in[0, \bar{\mu}]$, with

$$
c \equiv \frac{\left|\sigma_{2}\right|}{\left|v_{2}\right|}+\frac{\left|\sigma_{1}\right|}{\left|v_{1}\right|}>0, \quad \delta \equiv \frac{1}{2}\left(\frac{1}{v_{1}}-\frac{1}{v_{2}}\right)>0 \quad \text { and } \quad \bar{\mu} \equiv c\left\|A_{3}\right\|_{L^{\infty}} / \delta
$$

We set

$$
\varphi(x) \equiv \exp \left(\mu x\left(\begin{array}{cc}
1 / v_{1} & 0 \\
0 & 1 / v_{2}
\end{array}\right)\right) \psi(x)=\left(\begin{array}{c}
\mathrm{e}^{\mu x / v_{1}} \psi_{1} \\
\mathrm{e}^{\mu x / v_{2}} \psi_{2}
\end{array}\right),
$$

which transforms (28) into

$$
\left\{\begin{array}{l}
\varphi_{1}^{\prime}(x)+\frac{\sigma_{1}}{v_{1}} A_{3}(x) \mathrm{e}^{2 \mu \delta x} \varphi_{2}(x)=0 \\
\varphi_{1}(x)+\frac{\sigma_{2}}{v_{2}} A_{3}(x) \mathrm{e}^{-2 \mu \delta x} \varphi_{1}(x)=0 .
\end{array}\right.
$$

We then consider for $R>0$ that will be chosen sufficiently large, the fixed point problem in $(-\infty,-R)$

$$
\varphi(x)=\Upsilon_{\mu}[\varphi](x) \equiv\left(\begin{array}{c}
0 \\
1
\end{array}\right)-\int_{-\infty}^{x}\left(\begin{array}{c}
\frac{\sigma_{1}}{v_{1}} \varphi_{2}(y) A_{3}(y) \mathrm{e}^{2 \mu \delta y} \\
\frac{\sigma_{2}}{v_{2}} \varphi_{1}(y) A_{3}(y) \mathrm{e}^{-2 \mu \delta y}
\end{array}\right) d y
$$


For $0 \leq \mu \leq \bar{\mu}$, we shall solve (29) in the Banach space

$$
E_{\mu} \equiv\left\{\varphi=\left(\varphi_{1}, \varphi_{2}\right):(-\infty,-R) \rightarrow \mathbb{R}^{2}, \quad e^{2 \mu \delta|x|} \varphi_{1} \in L^{\infty}(-\infty,-R), \quad \varphi_{2} \in L^{\infty}(-\infty,-R)\right\}
$$

equipped with the norm

$$
\|\varphi\|_{E_{\mu}} \equiv\left\|\mathrm{e}^{2 \mu \delta|x|} \varphi_{1}\right\|_{L^{\infty}(-\infty,-R)}+\left\|\varphi_{2}\right\|_{L^{\infty}(-\infty,-R)} .
$$

In view of the easy estimates

$$
\left|\int_{-\infty}^{x} \frac{\sigma_{2}}{v_{2}} \varphi_{1}(y) A_{3}(y) \mathrm{e}^{-2 \mu \delta y}\right| \leq\left|\frac{\sigma_{2}}{v_{2}}\right|\left(\int_{-\infty}^{x}\left|A_{3}(y)\right| d y\right)\left\|\mathrm{e}^{2 \mu \delta|x|} \varphi_{1}\right\|_{L^{\infty}(-\infty,-R)}
$$

and

$$
\left|\int_{-\infty}^{x} \frac{\sigma_{1}}{v_{1}} \varphi_{2}(y) A_{3}(y) \mathrm{e}^{2 \mu \delta y}\right| \leq \mathrm{e}^{-2 \mu \delta|x|}\left|\frac{\sigma_{1}}{v_{1}}\right|\left(\int_{-\infty}^{x}\left|A_{3}(y)\right| d y\right)\left\|\varphi_{2}\right\|_{L^{\infty}(-\infty,-R)},
$$

we see that $\Upsilon_{\mu}: E_{\mu} \rightarrow E_{\mu}$ is a well-defined affine mapping. Moreover, if $R$ is chosen sufficiently large so that

$$
\left|\frac{\sigma_{1}}{v_{1}}\right|\left(\int_{-\infty}^{-R}\left|A_{3}(y)\right| d y\right) \leq \frac{1}{2} \quad \text { and } \quad\left|\frac{\sigma_{2}}{v_{2}}\right|\left(\int_{-\infty}^{-R}\left|A_{3}(y)\right| d y\right) \leq \frac{1}{2},
$$

the above inequalities show that $\Upsilon_{\mu}: E_{\mu} \rightarrow E_{\mu}$ is $\frac{1}{2}$-Lipschitz. Therefore, $\Upsilon_{\mu}: E_{\mu} \rightarrow E_{\mu}$ has a unique fixed point $\varphi_{\mu} \in E_{\mu}$. Furthermore,

$$
\left\|\varphi_{\mu}\right\|_{E_{\mu}}=\left\|\Upsilon_{\mu}\left[\varphi_{\mu}\right]\right\|_{E_{\mu}} \leq\left\|\Upsilon_{\mu}\left[\varphi_{\mu}\right]-\Upsilon_{\mu}[0]\right\|_{E_{\mu}}+\left\|\Upsilon_{\mu}[0]\right\|_{E_{\mu}} \leq \frac{1}{2}\left\|\varphi_{\mu}\right\|_{E_{\mu}}+1
$$

hence

$$
\left\|\varphi_{\mu}\right\|_{E_{\mu}}=\left\|\mathrm{e}^{2 \mu \delta|x|}\left(\varphi_{\mu}\right)_{1}\right\|_{L^{\infty}(-\infty,-R)}+\left\|\left(\varphi_{\mu}\right)_{2}\right\|_{L^{\infty}(-\infty,-R)} \leq 2
$$

We then set

$$
\psi_{\mu}^{-}(x) \equiv \exp \left(-\mu x\left(\begin{array}{cc}
1 / v_{1} & 0 \\
0 & 1 / v_{2}
\end{array}\right)\right) \varphi_{\mu}(x)=\left(\begin{array}{c}
\mathrm{e}^{-\mu x / v_{1}}\left(\varphi_{\mu}\right)_{1} \\
\mathrm{e}^{-\mu x / v_{2}}\left(\varphi_{\mu}\right)_{2}
\end{array}\right) .
$$

From (30), (31) and (32) and the fact that $\int_{-\infty}^{x}\left|A_{3}\right| d y \rightarrow 0$ as $x \rightarrow-\infty$, it follows that

$$
\psi_{\mu}^{-}(x)=\mathrm{e}^{-\mu x / v_{2}}\left[\left(\begin{array}{l}
0 \\
1
\end{array}\right)+o(1)\right] \quad \text { as } \quad x \rightarrow-\infty,
$$

with $o(1)$ uniform with respect to $\mu \in[0, \bar{\mu}]$. Following the same lines for $x \rightarrow+\infty$, we construct, for $0 \leq \mu \leq \bar{\mu}$, a solution $\psi_{\mu}^{+}$of (28) such that

$$
\psi_{\mu}^{+}(x)=\mathrm{e}^{-\mu x / v_{1}}\left[\left(\begin{array}{l}
1 \\
0
\end{array}\right)+o(1)\right] \quad \text { as } \quad x \rightarrow+\infty,
$$

with here again a uniform $o(1)$ for $\mu \in[0, \bar{\mu}]$.

In a second step, we use polar coordinates $\psi=\left(\psi_{1}, \psi_{2}\right)=\rho(\cos \theta, \sin \theta)$, and rewrite the system (28) under the form

$$
\left\{\begin{array}{l}
\rho^{\prime}=-\mu \rho\left(\frac{\cos ^{2}(\theta)}{v_{1}}+\frac{\sin ^{2}(\theta)}{v_{2}}\right)-\left(\frac{\sigma_{1}}{v_{1}}+\frac{\sigma_{2}}{v_{2}}\right) \rho A_{3} \\
\theta^{\prime}=\mu\left(\frac{1}{v_{1}}-\frac{1}{v_{2}}\right) \cos \theta \sin \theta+A_{3}\left(\frac{\sigma_{1}}{v_{1}} \sin ^{2} \theta-\frac{\sigma_{2}}{v_{2}} \cos ^{2} \theta\right) .
\end{array}\right.
$$

Note that the equation for $\theta$ does not involve $\rho$. We shall denote $\psi_{\mu}^{ \pm}=\rho_{\mu}^{ \pm}\left(\cos \theta_{\mu}^{ \pm}, \sin \theta_{\mu}^{ \pm}\right)$, and from , we see that we can choose $\theta_{\mu}^{-}$(resp. $\theta_{\mu}^{+}$) such that $\theta_{\mu}^{-}(-\infty)=\pi / 2\left(\right.$ resp. $\left.\theta_{\mu}^{+}(+\infty)=0\right)$, and that the mapping $[0, \bar{\mu}] \times(-\infty,-R] \ni(\mu, x) \mapsto \theta_{\mu}^{-}(x) \in \mathbb{R}\left(\operatorname{resp} .[0, \bar{\mu}] \times\left([+R,+\infty) \ni(\mu, x) \mapsto \theta_{\mu}^{+}(x) \in \mathbb{R}\right)\right.$ is uniformly continuous. We focus then on the second equation of the above system, namely

$$
\theta^{\prime}=\mu\left(\frac{1}{v_{1}}-\frac{1}{v_{2}}\right) \cos \theta \sin \theta+A_{3}\left(\frac{\sigma_{1}}{v_{1}} \sin ^{2} \theta-\frac{\sigma_{2}}{v_{2}} \cos ^{2} \theta\right) .
$$


The point is to show that for some $\mu>0$, there exists $m \in \mathbb{Z}$ such that $\theta_{\mu}^{-}=\theta_{\mu}^{+}+m \pi$ on $\mathbb{R}$. Since $\theta_{\mu}^{-}$and $\theta_{\mu}^{+}$solve (35), it is sufficient to show that $\theta_{\mu}^{-}(x)=\theta_{\mu}^{+}(x) \bmod \pi$ for some $x \in \mathbb{R}$, which will be done by using the intermediate value theorem in $[0, \bar{\mu}]$.

We consider first the case $\mu=0$. Then, we have for $x \in \mathbb{R}$,

$$
\int_{-\infty}^{x} A_{3}(y) d y=\int_{-\infty}^{x} \frac{\left(\theta_{0}^{-}\right)^{\prime}}{\frac{\sigma_{2}}{v_{2}} \cos ^{2} \theta_{0}^{-}-\frac{\sigma_{1}}{v_{1}} \sin ^{2} \theta_{0}^{-}} d y=\int_{\pi / 2}^{\theta_{0}^{-}(x)} \frac{d \Theta}{\frac{\sigma_{2}}{v_{2}} \cos ^{2} \Theta-\frac{\sigma_{1}}{v_{1}} \sin ^{2} \Theta} .
$$

The integrand in the right-hand side is $\pi$-periodic, even, of constant sign (since $\left.\sigma_{1} \sigma_{2}>0>v_{1} v_{2}\right)$ equal to $\epsilon \equiv \operatorname{sgn}\left(\sigma_{2} / v_{2}\right)=\operatorname{sgn}\left(\sigma_{1} / v_{1}\right)=-\operatorname{sgn}\left(\sigma_{2} / v_{2}\right)= \pm 1$, and has integral over one period equal to (using the change of variable $t=\tan \Theta)$

$\forall \alpha \in \mathbb{R}, \quad \int_{\alpha}^{\alpha+\pi} \frac{d \Theta}{\frac{\sigma_{2}}{v_{2}} \cos ^{2} \Theta-\frac{\sigma_{1}}{v_{1}} \sin ^{2} \Theta}=\int_{-\pi / 2}^{+\pi / 2} \frac{d \Theta}{\frac{\sigma_{2}}{v_{2}} \cos ^{2} \Theta-\frac{\sigma_{1}}{v_{1}} \sin ^{2} \Theta}=\int_{-\infty}^{+\infty} \frac{d t}{\frac{\sigma_{2}}{v_{2}}-\frac{\sigma_{1}}{v_{1}} t^{2}}=\epsilon \pi \sqrt{\frac{\left|v_{1} v_{2}\right|}{\sigma_{1} \sigma_{2}}}$.

Since, by assumption,

$$
\left|\int_{\mathbb{R}} A_{3}(y) d y\right|>\frac{\pi}{2} \sqrt{\frac{\left|v_{1} v_{2}\right|}{\sigma_{1} \sigma_{2}}}
$$

it follows that $\theta_{0}^{-}$has a finite limit at $+\infty$ which is such that

$$
\eta \equiv\left|\theta_{0}^{-}(+\infty)-\theta_{0}^{-}(-\infty)\right|-\frac{\pi}{2}=\left|\theta_{0}^{-}(+\infty)-\frac{\pi}{2}\right|-\frac{\pi}{2}>0
$$

Since $\theta_{\mu}^{-}$and $\theta_{\mu}^{+}$tend to $\pi / 2$ and 0 at $-\infty$ and $+\infty$ uniformly with respect to $[0, \bar{\mu}]$, we can choose some $r \gg 1$ such that, for $0 \leq \mu \leq \bar{\mu}$,

$$
\left|\theta_{\mu}^{-}(-r)-\frac{\pi}{2}\right| \leq \frac{\pi}{4}, \quad \quad\left|\theta_{\mu}^{+}(+r)\right| \leq \frac{1}{10} \min (\eta, \pi) \quad \text { and } \quad\left|\theta_{0}^{-}(+r)-\theta_{0}^{-}(+\infty)\right| \leq \frac{\eta}{10} .
$$

It then follows that

$$
\left|\theta_{0}^{-}(+r)-\theta_{0}^{+}(+r)-\frac{\pi}{2}\right|=\left|\left(\theta_{0}^{-}(+r)-\theta_{0}^{-}(+\infty)\right)+\left(\theta_{0}^{-}(+\infty)-\frac{\pi}{2}\right)-\theta_{0}^{+}(+r)\right| \geq \frac{\pi}{2}+\frac{4 \eta}{5},
$$

thus $\theta_{0}^{-}(+r)-\theta_{0}^{+}(+r) \notin[0, \pi]$.

Now, we focus on the case where $\mu=\bar{\mu}$. We have

$$
-c\left\|A_{3}\right\|_{L^{\infty}} \leq \frac{d \theta_{\bar{\mu}}^{-}}{d x}-2 \delta \bar{\mu} \cos \theta_{\bar{\mu}}^{-} \sin \theta_{\bar{\mu}}^{-} \leq c\left\|A_{3}\right\|_{L^{\infty}}
$$

Denoting $\underline{\theta}$ and $\bar{\theta}$ the solutions of

$$
\left\{\begin{array}{lrl}
\frac{d \underline{\theta}}{d x}=\underline{F}(\underline{\theta}) \equiv 2 \delta \bar{\mu} \cos \underline{\theta} \sin \underline{\theta}-c\left\|A_{3}\right\|_{L^{\infty}}=\delta \bar{\mu}(-1+\sin (2 \underline{\theta})) & \text { with } \quad \underline{\theta}(-r)=\theta_{\mu}^{-}(-r) \\
\frac{d \bar{\theta}}{d x}=\bar{F}(\bar{\theta}) \equiv 2 \delta \bar{\mu} \cos \bar{\theta} \sin \bar{\theta}+c\left\|A_{3}\right\|_{L^{\infty}}=\delta \bar{\mu}(1+\sin (2 \bar{\theta})) & \text { with } \quad \bar{\theta}(-r)=\theta_{\mu}^{-}(-r) .
\end{array}\right.
$$

(for which we have global existence) we then have

$$
\underline{\theta} \leq \theta_{\bar{\mu}}^{-} \leq \bar{\theta} \quad \text { in }[-r,+r] .
$$

It is immediate that $\bar{F}$ is positive on the interval $(\pi / 4,3 \pi / 4)$ and vanishes at its right end; similarly, $\underline{F}$ is negative on the interval $(\pi / 4,3 \pi / 4)$ and vanishes at its left end. We then infer, since $\theta_{\bar{\mu}}^{-}(-r) \in(\pi / 4,3 \pi / 4)$, that on $[-r,+r]$,

Consequently,

$$
\frac{\pi}{4} \leq \theta^{-} \leq \theta_{\bar{\mu}}^{-} \leq \theta^{+} \leq \frac{3 \pi}{4}
$$

$$
\left|\theta_{\bar{\mu}}^{-}(+r)-\theta_{\bar{\mu}}^{+}(+r)-\frac{\pi}{2}\right| \leq\left|\theta_{\bar{\mu}}^{-}(+r)-\frac{\pi}{2}\right|+\left|\theta_{\bar{\mu}}^{+}(+r)\right| \leq \frac{\pi}{4}+\frac{\pi}{10}<\frac{\pi}{2}
$$

and $\theta_{\bar{\mu}}^{-}(+r)-\theta_{\bar{\mu}}^{+}(+r) \in(0, \pi)$. Since the mapping $[0, \bar{\mu}] \ni \mu \mapsto \theta_{\bar{\mu}}^{-}(+r)-\theta_{\bar{\mu}}^{+}(+r) \in \mathbb{R}$ is continuous (up to $\mu=0)$, it follows from the intermediate value theorem that for some $0<\mu<\bar{\mu}, \theta_{\bar{\mu}}^{-}(+r)-\theta_{\bar{\mu}}^{+}(+r)$ is an integer multiple of $\pi$. For this value of $\mu, \psi_{\mu}^{+}$is collinear to $\psi_{\mu}^{-}$, and we have then obtained a solution $\psi=\psi_{\mu}^{-}=K \psi_{\mu}^{+}$(for some $K \in \mathbb{R}^{*}$ ) to (28) which tends to 0 at $\pm \infty$ exponentially fast. 


\subsubsection{Proof of (iii)}

We first recall that if $v_{1}+v_{2}=0$, then $\gamma=\gamma_{*}$ and the estimates (iii) and (iv) come from Proposition 6 . We thus assume $v_{1}+v_{2} \neq 0$. We introduce the variables (close to the characteristic variables)

$$
\hat{x} \equiv\left(\frac{1}{v_{1}}-\frac{1}{v_{2}}\right) x \quad \text { and } \quad \hat{t} \equiv 2 t-\left(\frac{1}{v_{1}}+\frac{1}{v_{2}}\right) x,
$$

and denote

$$
\hat{A}_{j}(\hat{t}, \hat{x})=A_{j}(t, x) \quad j=1,2, \quad \hat{A}_{3}(\hat{x}) \equiv A_{3}(x) .
$$

Writing (5) in terms of $\hat{A}=\left(\hat{A}_{1}, \hat{A}_{2}\right)$, we infer the system in $(\hat{t}, \hat{x})$

$$
\left\{\begin{array}{l}
\partial_{\hat{t}} \hat{A}_{1}+\partial_{\hat{x}} \hat{A}_{1}+\sigma_{1} \frac{v_{2}}{v_{2}-v_{1}} \hat{A}_{3} \hat{A}_{2}=0 \\
\partial_{\hat{t}} \hat{A}_{2}-\partial_{\hat{x}} \hat{A}_{2}+\sigma_{2} \frac{v_{1}}{v_{1}-v_{2}} \hat{A}_{3} \hat{A}_{1}=0
\end{array}\right.
$$

This system in variables $(\hat{t}, \hat{x})$ is of the type $(5)$ with $\left(\sigma_{1}, \sigma_{2}\right)$ replaced by $\left(\hat{\sigma}_{1}, \hat{\sigma}_{2}\right) \equiv\left(\sigma_{1} \frac{v_{2}}{v_{2}-v_{1}}, \sigma_{2} \frac{v_{1}}{v_{1}-v_{2}}\right)$, for which $\hat{\sigma}_{1} \hat{\sigma}_{2}>0$. Thus, we may apply the result of Proposition 6 (forward and backward in time) to get

$$
\|\hat{A}(\hat{t})\|_{L^{\infty}} \leq C\|\hat{A}(\hat{t}=0)\|_{L^{\infty}} \exp \left(|\hat{t}| \sqrt{\sigma_{1} \sigma_{2}} \frac{\sqrt{\left|v_{1} v_{2}\right|}}{\left|v_{1}-v_{2}\right|}\left\|\hat{A}_{3}\right\|_{L^{\infty}}\right) .
$$

We recall that we have assumed $v_{1}+v_{2} \neq 0$, thus we can set

$$
\hat{v} \equiv \frac{2 v_{1} v_{2}}{v_{1}+v_{2}} \in \mathbb{R}^{*}
$$

so that the line $\{\hat{t}=0\}$ is the line $\{t=x / \hat{v}\}$. In order to be able to use (36), we need to estimate

$$
\|\hat{A}(\hat{t}=0)\|_{L^{\infty}}=\|\theta \mapsto A(\theta, \theta \hat{v})\|_{L^{\infty}}
$$

Since $v_{1}>0>v_{2}$

$$
\frac{1}{\hat{v}}=\frac{1}{v_{1}}+\frac{1}{v_{2}} \in\left(\frac{1}{v_{2}}, \frac{1}{v_{1}}\right)
$$

and then $\hat{v}<v_{2}$ (if $v_{1}>-v_{2}$ ) or $\hat{v}>v_{1}$ (if $v_{1}<-v_{2}$ ). We first treat the case $\hat{v}>v_{1}$. By assumption, $A_{3} \in L^{1}$, thus there exists $R>0$ such that

$$
\sqrt{\frac{\sigma_{1} \sigma_{2}}{\left|v_{1} v_{2}\right|}} \int_{|y|>R}\left|A_{3}\right|(y) d y \leq \frac{1}{2} .
$$

We shall follow the first lines of the proof of Proposition 5 to obtain a large time estimate for

$$
\mathrm{N}(t) \equiv\left\|A_{1}(t)\right\|_{L^{\infty}\left(\left\{x \geq R+t v_{1}\right\}\right)}+\sqrt{\frac{\sigma_{1}}{\sigma_{2}}\left|\frac{v_{2}}{v_{1}}\right|}\left\|A_{2}(t)\right\|_{L^{\infty}\left(\left\{x \geq R+t v_{1}\right\}\right)} .
$$

Let us fix $0 \leq t^{\prime} \leq t$ and $x \geq R+t^{\prime} v_{1}$. Note that for $0 \leq \tau \leq t^{\prime}$, we have

$x-\left(t^{\prime}-\tau\right) v_{1} \geq\left(R+t^{\prime} v_{1}\right)-\left(t^{\prime}-\tau\right) v_{1}=R+\tau v_{1} \quad$ and $\quad x-\left(t^{\prime}-\tau\right) v_{2} \geq x \geq R+t^{\prime} v_{1} \geq R+\tau v_{1}$.

Hence, from (10), we infer

$$
\begin{aligned}
\left|A_{1}\right|\left(t^{\prime}, x\right) & \leq\left|A_{1}^{\text {in }}\left(x-t^{\prime} v_{1}\right)\right|+\left|\sigma_{1}\right| \int_{0}^{t^{\prime}}\left\|A_{2}(\tau, \cdot)\right\|_{L^{\infty}\left(\left\{y \geq R+\tau v_{1}\right\}\right)}\left|A_{3}\right|\left(x-\left(t^{\prime}-\tau\right) v_{1}\right) d \tau \\
& \leq\left\|A_{1}^{\text {in }}\right\|_{L^{\infty}}+\frac{\left|\sigma_{1}\right|}{\left|v_{1}\right|}\left(\sup _{0 \leq \tau \leq t}\left\|A_{2}(\tau, \cdot)\right\|_{L^{\infty}\left(\left\{y \geq R+\tau v_{1}\right\}\right)}\right)\left(\int_{R}^{+\infty}\left|A_{3}\right|(y) d y\right)
\end{aligned}
$$

and similarly

$$
\left|A_{2}\right|\left(t^{\prime}, x\right) \leq\left\|A_{2}^{\mathrm{in}}\right\|_{L^{\infty}}+\frac{\left|\sigma_{2}\right|}{\left|v_{2}\right|}\left(\sup _{0 \leq \tau \leq t}\left\|A_{1}(\tau, \cdot)\right\|_{L^{\infty}\left(\left\{y \geq R+\tau v_{1}\right\}\right)}\right)\left(\int_{R}^{+\infty}\left|A_{3}\right|(y) d y\right) .
$$


Consequently, using (37), we deduce that for $0 \leq t^{\prime} \leq t$,

$$
\mathrm{N}\left(t^{\prime}\right) \leq C\left\|A^{\mathrm{in}}\right\|_{L^{\infty}}+\frac{1}{2} \sup _{0 \leq \tau \leq t} \mathrm{~N}(\tau)
$$

from which it comes, for any $t \geq 0$,

$$
\mathrm{N}(t) \leq \sup _{0 \leq \tau \leq t} \mathrm{~N}(\tau) \leq 2 C\left\|A^{\mathrm{in}}\right\|_{L^{\infty}} .
$$

Since $\hat{v}>v_{1}$, for $\theta \geq R /\left(\hat{v}-v_{1}\right)$, we have $\theta \hat{v} \geq R+\theta v_{1}$. Therefore, the above estimate yields in particular

$$
\|\theta \mapsto A(\theta, \theta \hat{v})\|_{L^{\infty}\left(\left\{\theta \geq R /\left(\hat{v}-v_{1}\right)\right\}\right)} \leq C\left\|A^{\mathrm{in}}\right\|_{L^{\infty}} .
$$

In the case $\hat{v}<v_{2}<0$, we deduce by similar arguments (computing the $L^{\infty}$ norms in $\left\{y \leq-R+t v_{2}\right\}$ ) that

$$
\|\theta \mapsto A(\theta, \theta \hat{v})\|_{L^{\infty}\left(\left\{\theta \geq R /\left(v_{2}-\hat{v}\right)\right\}\right)} \leq C\left\|A^{\mathrm{in}}\right\|_{L^{\infty}} .
$$

The estimates for $\theta \ll-1$ are derived in the same way. Hence, there exists some $\Theta>0$, depending only on $R, v_{1}$ and $v_{2}$, such that

$$
\|\theta \mapsto A(\theta, \theta \hat{v})\|_{L^{\infty}(\{|\theta| \geq \Theta\})} \leq C\left\|A^{\text {in }}\right\|_{L^{\infty}} .
$$

Since $A_{3} \in L^{\infty}$, the Cauchy problem (5) is locally well-posed in $L^{\infty}$ and it follows that

$$
\|\theta \mapsto A(\theta, \theta \hat{v})\|_{L^{\infty}(\{|\theta| \leq \Theta\})} \leq C\left\|A^{\mathrm{in}}\right\|_{L^{\infty}}
$$

for some constant $C$ depending on $A_{3}, \sigma_{1}, \sigma_{2}, v_{1}$ and $v_{2}$. Collecting the above inequalities, it comes

$$
\|\hat{A}(\hat{t}=0)\|_{L^{\infty}}=\|\theta \mapsto A(\theta, \theta \hat{v})\|_{L^{\infty}} \leq C\left\|A^{\mathrm{in}}\right\|_{L^{\infty}} .
$$

Inserting this into (36) yields for $t \geq 0$ and $x \in \mathbb{R}$

$$
|A(t, x)|_{\infty}=|\hat{A}(\hat{t}, \hat{x})|_{\infty} \leq C\left\|A^{\mathrm{in}}\right\|_{L^{\infty}} \exp \left(\left|2 t-\frac{x}{\hat{v}}\right| \frac{\gamma_{*}}{2}\right) .
$$

This estimate is sufficient to show that eigenvalues for (7) (if they exist) lie in $\left\{\operatorname{Im} \geq-\gamma_{*}\right\}$. Indeed, assume that $\lambda \in \mathbb{C}$ is an eigenvalue for (7) with corresponding eigenvector $\psi$, so that $A(t, x)=\mathrm{e}^{i \lambda t} \psi(x)$ solves (5). Then, (39) yields, for any $x \in \mathbb{R}$,

$$
|A(t, x)|_{\infty}=\mathrm{e}^{-t \operatorname{Im} \lambda}|\psi(x)|_{\infty} \leq C\|\psi\|_{L^{\infty}} \exp \left(\left|2 t-\frac{x}{\hat{v}}\right| \frac{\gamma_{*}}{2}\right) .
$$

Choosing $x=x_{0} \in \mathbb{R}$ such that $2\left|\psi\left(x_{0}\right)\right|_{\infty} \geq\|\psi\|_{L^{\infty}}$, this gives

$$
\mathrm{e}^{-t \operatorname{Im} \lambda} \leq 2 C \exp \left(\left|2 t-\frac{x_{0}}{\hat{v}}\right| \frac{\gamma_{*}}{2}\right)
$$

which yields the result letting $t \rightarrow+\infty$.

To prove (iii), we use (39) only for $x \in[-R,+R]$ :

$$
\|A(t)\|_{L^{\infty}(-R,+R)} \leq C_{R}\left\|A^{\mathrm{in}}\right\|_{L^{\infty}} \mathrm{e}^{t \gamma_{*}} .
$$

We now derive an estimate for

$$
N(t) \equiv\left\|A_{1}\right\|_{L^{\infty}((0, t) \times(R,+\infty))}+\sqrt{\frac{\sigma_{1}}{\sigma_{2}}\left|\frac{v_{2}}{v_{1}}\right|}\left\|A_{2}\right\|_{L^{\infty}((0, t) \times(R,+\infty))} .
$$

For $x \geq R$ and $0 \leq t^{\prime} \leq t$, we have, by (10) and since $v_{2}<0$,

$$
\left|A_{2}\right|\left(t^{\prime}, x\right) \leq\left\|A_{2}^{\mathrm{in}}\right\|_{L^{\infty}}+\frac{\left|\sigma_{2}\right|}{\left|v_{2}\right|}\left\|A_{1}\right\|_{L^{\infty}((0, t) \times(R,+\infty))} \int_{R}^{+\infty}\left|A_{3}\right|(y) d y .
$$

Furthermore, (38) gives in particular

$$
\left\|A_{1}\right\|_{L^{\infty}\left(\left\{0 \leq t^{\prime} \leq t, x \geq R+t^{\prime} v_{1}\right\}\right)} \leq C\left\|A^{\mathrm{in}}\right\|_{L^{\infty}}
$$


Let now $0 \leq t^{\prime} \leq t$ and $R \leq x \leq R+t^{\prime} v_{1}$. By the method of characteristics, we obtain

$$
A_{1}\left(t^{\prime}, x\right)=A_{1}\left(t^{\prime}-\frac{x-R}{v_{1}}, R\right)-\sigma_{1} \int_{t^{\prime}-\frac{x-R}{v_{1}}}^{t^{\prime}} A_{2}\left(\tau, x-\left(t^{\prime}-\tau\right) v_{1}\right) A_{3}\left(x-\left(t^{\prime}-\tau\right) v_{1}\right) d \tau .
$$

We use (40) to bound the first term $\left(t^{\prime}-(x-R) / v_{1} \leq t^{\prime} \leq t\right)$, and since $x-\left(t^{\prime}-\tau\right) v_{1} \geq R$ in the integral, we obtain, for $R \leq x \leq R+t^{\prime} v_{1}$,

$$
\left\|A_{1}\right\|_{L^{\infty}\left(\left\{0 \leq t^{\prime} \leq t, R \leq x \leq R+t^{\prime} v_{1}\right\}\right)} \leq C_{R}\left\|A^{\mathrm{in}}\right\|_{L^{\infty}} \mathrm{e}^{t \gamma_{*}}+\frac{\left|\sigma_{1}\right|}{v_{1}}\left\|A_{2}\right\|_{L^{\infty}((0, t) \times(R,+\infty))} \int_{R}^{+\infty}\left|A_{3}\right|(y) d y .
$$

the combination of (41), (42) and (43) yields

$$
N(t) \leq C_{R}\left\|A^{\mathrm{in}}\right\|_{L^{\infty}} \mathrm{e}^{t \gamma_{*}}+N(t)\left(\sqrt{\frac{\sigma_{1} \sigma_{2}}{\left|v_{1} v_{2}\right|}} \int_{R}^{+\infty}\left|A_{3}\right|(y) d y\right)^{2} .
$$

Possibly taking $R$ larger if necessary, we can assume the term in parenthesis $\leq 1 / \sqrt{2}$, so that

$$
N(t) \leq 2 C_{R}\left\|A^{\text {in }}\right\|_{L^{\infty}} \mathrm{e}^{t \gamma_{*}} .
$$

The estimate for $x \leq-R$ follows the same lines, and combining with (40), we infer as claimed

$$
\|A(t)\|_{L^{\infty}} \leq C\left\|A^{\mathrm{in}}\right\|_{L^{\infty}} \mathrm{e}^{t \gamma_{*}}
$$

\subsection{Proof of Proposition 9}

We recall that $\sigma_{1} \sigma_{2}>0$. Possibly changing $x$ for $-x$, we may, without loss of generality, assume that $v_{1}>0$ and $v_{2}>0$. Moreover, if necessary, we may exchange the indices 1 and 2 so that $v_{2} \geq v_{1}>0$.

\subsubsection{Proof of case (i)}

In case $(i), v_{1}=v_{2}>0$ will be denoted $v$. We can notice that the explicit computation yielding (22) is still valid in this case, thus

$$
\left\{\begin{aligned}
A_{1}(t, x)= & A_{1}^{\mathrm{in}}(x-t v) \cosh \left(\frac{\sqrt{\sigma_{1} \sigma_{2}}}{v} \int_{x-t v}^{x} A_{3}(y) d y\right) \\
& -\operatorname{sgn}\left(\sigma_{1}\right) \sqrt{\frac{\sigma_{1}}{\sigma_{2}}} A_{2}^{\mathrm{in}}(x-t v) \sinh \left(\frac{\sqrt{\sigma_{1} \sigma_{2}}}{v} \int_{x-t v}^{x} A_{3}(y) d y\right) \\
A_{2}(t, x)= & A_{2}^{\mathrm{in}}(x-t v) \cosh \left(\frac{\sqrt{\sigma_{1} \sigma_{2}}}{v} \int_{x-t v}^{x} A_{3}(y) d y\right) \\
& -\operatorname{sgn}\left(\sigma_{1}\right) \sqrt{\frac{\sigma_{2}}{\sigma_{1}}} A_{1}^{\mathrm{in}}(x-t v) \sinh \left(\frac{\sqrt{\sigma_{1} \sigma_{2}}}{v} \int_{x-t v}^{x} A_{3}(y) d y\right)
\end{aligned}\right.
$$

and $(i)$ follows easily.

\subsubsection{Proof of case (ii)}

Let $(T, X) \in \mathbb{R}^{+} \times \mathbb{R}$, and assume that $A$ solves (5). We recall that $A^{\text {in }} \in \mathcal{C}_{b}(\mathbb{R})$, hence $A \in \mathcal{C}\left(\mathbb{R}_{+} \times \mathbb{R}, \mathbb{C}\right)$. Due to the finite speeds of propagation $v_{2} \geq v_{1}>0$, the value of $A(T, X)$ will depend only on $A_{3}$ and $A^{\text {in }}$ in $\left[X-T v_{2}, X\right]$. We wish to prove, for some constant $C>0$ depending only on $\sigma_{1}, \sigma_{2}, v_{1}$ and $v_{2}$, the pointwise estimate

$$
|A(T, X)|_{\infty} \leq C\left\|A^{\mathrm{in}}\right\|_{\mathcal{C}\left(\left[X-T v_{2}, X\right]\right)} \exp \left(\sqrt{\frac{\sigma_{1} \sigma_{2}}{v_{1} v_{2}}} \int_{X-T v_{2}}^{X}\left|A_{3}\right|(y) d y\right) .
$$

For some given $r \in \mathbb{N}^{*}$ (that will tend to $+\infty$ ), we divide the interval $\left[X-T v_{2}, X\right]$ into $r$ subintervals $\left[x^{j}, x^{j+1}\right], 0 \leq j<r$, with $x^{j} \equiv X-T v_{2}(1-j / r), j \in \mathbb{Z}$ (we omit the dependency on $r$ in the notations). Since $A_{3} \in L_{\text {loc }}^{1}(\mathbb{R})$, there exists $r_{0} \in \mathbb{N}^{*}$ (depending on $T$ and $X$ ) such that, if $r \geq r_{0}$,

$$
\forall 0 \leq j<r, \quad \alpha^{j} \equiv \sqrt{\frac{\sigma_{1} \sigma_{2}}{v_{1} v_{2}}} \int_{x^{j}}^{x^{j+1}}\left|A_{3}\right|(y) d y<1 .
$$


On each subinterval $\left(x^{j}, x^{j+1}\right)(0 \leq j<r), A$ solves, in the space-time trapezoid

$$
\mathcal{D}^{j} \equiv\left\{(t, x) \in \mathbb{R}^{+} \times\left(x^{j}, x^{j+1}\right), x-t v_{2} \geq X-T v_{2}\right\}
$$

the boundary value problem

$$
\left\{\begin{array}{l}
\partial_{t} A_{1}+v_{1} \partial_{x} A_{1}+\sigma_{1} A_{3} A_{2}=0 \\
\partial_{t} A_{2}+v_{2} \partial_{x} A_{2}+\sigma_{2} \bar{A}_{3} A_{1}=0,
\end{array} \quad \text { for } \quad(t, x) \in \mathcal{D}^{j}\right.
$$

with the boundary conditions ( $i n c e v_{2} \geq v_{1}>0$, they are naturally on the left-hand side of the domain and at the initial time)

$$
\begin{cases}A\left(t, x=x^{j}\right)=A\left(t, x=x^{j}\right), & 0 \leq t \leq T, \\ A(t=0, x)=A^{\text {in }}(x), & x^{j} \leq x \leq x^{j+1} .\end{cases}
$$

Using the method of characteristics, we find for $(t, x) \in \mathcal{D}^{j}$ :

$$
\begin{aligned}
A_{1}(t, x)= & \mathbf{1}_{x \geq x^{j}+t v_{1}}\left\{A_{1}^{\mathrm{in}}\left(x-t v_{1}\right)-\sigma_{1} \int_{0}^{t} A_{3}\left(x-(t-\tau) v_{1}\right) A_{2}\left(\tau, x-(t-\tau) v_{1}\right) d \tau\right\} \\
& +\mathbf{1}_{x \leq x^{j}+t v_{1}}\left\{A_{1}\left(t-\frac{x-x^{j}}{v_{1}}, x^{j}\right)-\sigma_{1} \int_{t-\frac{x-x^{j}}{v_{1}}}^{t} A_{3}\left(x-(t-\tau) v_{1}\right) A_{2}\left(\tau, x-(t-\tau) v_{1}\right) d \tau\right\} \\
A_{2}(t, x)= & \mathbf{1}_{x \geq x^{j}+t v_{2}}\left\{A_{2}^{\mathrm{in}}\left(x-t v_{2}\right)-\sigma_{2} \int_{0}^{t} \bar{A}_{3}\left(x-(t-\tau) v_{2}\right) A_{1}\left(\tau, x-(t-\tau) v_{2}\right) d \tau\right\} \\
& +\mathbf{1}_{x \leq x^{j}+t v_{2}}\left\{A_{2}\left(t-\frac{x-x^{j}}{v_{2}}, x^{j}\right)-\sigma_{2} \int_{t-\frac{x-x^{j}}{v_{2}}}^{t} \bar{A}_{3}\left(x-(t-\tau) v_{2}\right) A_{1}\left(\tau, x-(t-\tau) v_{2}\right) d \tau\right\} .
\end{aligned}
$$

Denoting $t^{j} \equiv T-\left(X-x^{j}\right) / v_{2}$ for $0 \leq j \leq r$, this yields the inequalities $\left(\right.$ since $\left.v_{2} \geq v_{1}>0\right)$

$$
\begin{aligned}
&\left\|A_{1}\right\|_{\mathcal{C}\left(\mathcal{D}^{j}\right)} \leq \max \left\{\left\|A_{1}^{\mathrm{in}}\right\|_{\mathcal{C}\left(\left[x^{j}, x^{j+1}\right]\right)}+\frac{\left|\sigma_{1}\right|}{v_{1}}\left\|A_{2}\right\|_{\mathcal{C}\left(\mathcal{D}^{j}\right)} \int_{x^{j}}^{x^{j+1}}\left|A_{3}\right|(y) d y\right.\left.\left\|A_{1}\left(\cdot, x^{j}\right)\right\|_{\mathcal{C}\left(\left[0, t^{j}\right]\right)}+\frac{\left|\sigma_{1}\right|}{v_{1}}\left\|A_{2}\right\|_{\mathcal{C}\left(\mathcal{D}^{j}\right)} \int_{x^{j}}^{x^{j+1}}\left|A_{3}\right|(y) d y\right\} \\
&= \max \left\{\left\|A_{1}^{\mathrm{in}}\right\|_{\mathcal{C}\left(\left[x^{j}, x^{j+1}\right]\right)} ;\left\|A_{1}\left(\cdot, x^{j}\right)\right\|_{\mathcal{C}\left(\left[0, t^{j}\right]\right)}\right\}+\frac{\left|\sigma_{1}\right|}{v_{1}}\left\|A_{2}\right\|_{\mathcal{C}\left(\mathcal{D}^{j}\right)} \int_{x^{j}}^{x^{j+1}}\left|A_{3}\right|(y) d y \\
&\left\|A_{2}\right\|_{\mathcal{C}\left(\mathcal{D}^{j}\right)} \leq \max \left\{\left\|A_{2}^{\mathrm{in}}\right\|_{\mathcal{C}\left(\left[x^{j}, x^{j+1}\right]\right)}+\frac{\left|\sigma_{2}\right|}{v_{2}}\left\|A_{1}\right\|_{\mathcal{C}\left(\mathcal{D}^{j}\right)} \int_{x^{j}}^{x^{j+1}}\left|A_{3}\right|(y) d y ;\right. \\
&\left.\qquad A_{2}\left(\cdot, x^{j}\right)\left\|_{\mathcal{C}\left(\left[0, t^{j}\right]\right)}+\frac{\left|\sigma_{2}\right|}{v_{2}}\right\| A_{1} \|_{\mathcal{C}\left(\mathcal{D}^{j}\right)} \int_{x^{j}}^{x^{j+1}}\left|A_{3}\right|(y) d y\right\} \\
&=\max \left\{\left\|A_{2}^{\mathrm{in}}\right\|_{\mathcal{C}\left(\left[x^{j}, x^{j+1}\right]\right)} ;\left\|A_{2}\left(\cdot, x^{j}\right)\right\|_{\mathcal{C}\left(\left[0, t^{j}\right]\right)}\right\}+\frac{\left|\sigma_{2}\right|}{v_{2}}\left\|A_{1}\right\|_{\mathcal{C}\left(\mathcal{D}^{j}\right)} \int_{x^{j}}^{x^{j+1}}\left|A_{3}\right|(y) d y .
\end{aligned}
$$

Setting, for $0 \leq j \leq r$,

$$
\left\{\begin{array}{l}
\mathfrak{a}_{1}^{j} \equiv \max \left\{\left\|A_{1}^{\mathrm{in}}\right\|_{\mathcal{C}\left(\left[X-T v_{2}, X\right]\right)} ;\left\|A_{1}\right\|_{\mathcal{C}\left(\mathcal{D}^{j}\right)}\right\} \\
\mathfrak{a}_{2}^{j} \equiv \sqrt{\frac{\sigma_{1} v_{2}}{\sigma_{2} v_{1}}} \max \left\{\left\|A_{2}^{\mathrm{in}}\right\|_{\mathcal{C}\left(\left[X-T v_{2}, X\right]\right)} ;\left\|A_{2}\right\|_{\mathcal{C}\left(\mathcal{D}^{j}\right)}\right\}
\end{array}\right.
$$

we infer from (48) and (49) that for $0 \leq j \leq r$,

$$
\left\{\begin{array}{l}
\mathfrak{a}_{1}^{j} \leq \mathfrak{a}_{1}^{j-1}+\alpha^{j} \mathfrak{a}_{2}^{j}, \\
\mathfrak{a}_{2}^{j} \leq \mathfrak{a}_{2}^{j-1}+\alpha^{j} \mathfrak{a}_{1}^{j} .
\end{array}\right.
$$


As a consequence, the quantity

$$
\mathfrak{a}^{j} \equiv \mathfrak{a}_{1}^{j}+\mathfrak{a}_{2}^{j}
$$

satisfies (in view of (45))

$$
\mathfrak{a}^{j} \leq \frac{\mathfrak{a}^{j-1}}{1-\alpha^{j}}
$$

Therefore, for some constant $C>0$ depending only on $\sigma_{1}, \sigma_{2}, v_{1}$ and $v_{2}$,

$$
\frac{1}{C}|A(T, X)|_{\infty} \leq \mathfrak{a}^{r} \leq \mathfrak{a}^{0} \times\left(\prod_{j=1}^{r}\left(1-\alpha^{j}\right)\right)^{-1}
$$

Letting $r \rightarrow+\infty$, since $\alpha^{j} \rightarrow 0$ uniformly for $0 \leq j \leq r$, we have

$$
\left(\prod_{j=1}^{r}\left(1-\alpha^{j}\right)\right)^{-1}=\exp \left(-\sum_{j=1}^{r} \ln \left(1-\alpha^{j}\right)\right) \rightarrow \exp \left(\sqrt{\frac{\sigma_{1} \sigma_{2}}{v_{1} v_{2}}} \int_{X-T v_{2}}^{X}\left|A_{3}\right|(y) d y\right) .
$$

Moreover, as $r \rightarrow+\infty$,

$$
\mathfrak{a}^{0} \rightarrow\left\|A_{1}^{\mathrm{in}}\right\|_{\mathcal{C}\left(\left[X-T v_{2}, X\right]\right)}+\sqrt{\frac{\sigma_{1} v_{2}}{\sigma_{2} v_{1}}}\left\|A_{2}^{\mathrm{in}}\right\|_{\mathcal{C}\left(\left[X-T v_{2}, X\right]\right)},
$$

thus (44) follows, and hence statement (ii).

\subsubsection{Proof of case (iii)}

We consider here $A_{3}$ satisfying hypothesis (13). It is sufficient to show that if $(T, X) \in \mathbb{R}^{+} \times \mathbb{R}^{-}$and $A^{\text {in }} \in \mathcal{C}_{c}\left(\mathbb{R}, \mathbb{R}^{2}\right)$ is such that

$$
0 \leq A_{1}^{\text {in }} \leq 1, \quad 0 \leq A_{2}^{\text {in }} \leq \sqrt{\frac{\sigma_{1} v_{2}}{\sigma_{2} v_{1}}} \quad A_{1}^{\text {in }}=1, \quad A_{2}^{\text {in }}=\sqrt{\frac{\sigma_{1} v_{2}}{\sigma_{2} v_{1}}} \quad \text { in } \quad\left[X-T v_{2}, X\right]
$$

and $A_{1}^{\text {in }}$ and $A_{2}^{\text {in }}$ are nondecreasing in $\mathbb{R}^{-}$, then

$$
|A(T, X)|_{\infty} \geq c \exp \left(\frac{v_{1}}{v_{2}} \sqrt{\frac{\sigma_{1} \sigma_{2}}{v_{1} v_{2}}} \int_{X-T v_{2}}^{X}\left|A_{3}\right|(y) d y\right)
$$

for some constant $c>0$ depending only on $\sigma_{1}, \sigma_{2}, v_{1}$ and $v_{2}$. Indeed, (iii) will then follow from the fact that $A_{3}$ vanishes in $[1,+\infty)$, hence

$$
\sup _{x \leq 0} \int_{x-T v_{1}}^{x}\left|A_{3}\right|(y) d y \geq \sup _{x \in \mathbb{R}} \int_{x-T v_{1}}^{x}\left|A_{3}\right|(y) d y-\int_{0}^{1}\left|A_{3}\right|(y) d y .
$$

We recall that (10) is a fixed point problem $A=\Upsilon[A]$, where $\Upsilon=\left(\Upsilon_{1}, \Upsilon_{2}\right)$,

$$
\left\{\begin{array}{l}
\Upsilon_{1}[A](t, x) \equiv A_{1}^{\mathrm{in}}\left(x-t v_{1}\right)-\sigma_{1} \int_{0}^{t} A_{2}\left(\tau, x-(t-\tau) v_{1}\right) A_{3}\left(x-(t-\tau) v_{1}\right) d \tau \\
\Upsilon_{2}[A](t, x) \equiv A_{2}^{\mathrm{in}}\left(x-t v_{2}\right)-\sigma_{2} \int_{0}^{t} A_{1}\left(\tau, x-(t-\tau) v_{2}\right) A_{3}\left(x-(t-\tau) v_{2}\right) d \tau
\end{array}\right.
$$

The solution $A$ is real-valued since $A^{\text {in }}$ and $A_{3}$ are. From (13) (recall that $\sigma_{1} \sigma_{2}>0$ ), $-\sigma_{1} A_{3}$ and $-\sigma_{2} A_{3}$ are nonnegative and nondecreasing in $\mathbb{R}^{-}$. Moreover, $A_{1}^{\text {in }}$ and $A_{2}^{\text {in }}$ are nonnegative and nondecreasing in $\mathbb{R}^{-}$. As a consequence, if $A_{1}(t)$ and $A_{2}(t)$ are nonnegative and nondecreasing in $\mathbb{R}^{-}$for every $t \geq 0$, then $\Upsilon_{1}[A](t)$ and $\Upsilon_{2}[A](t)$ are also nonnegative and nondecreasing in $\mathbb{R}^{-}$for every $t \geq 0$ (since $0 \leq v_{1}<v_{2}$, thus for $\left.0 \leq \tau \leq t, x-(t-\tau) v_{1} \leq x-(t-\tau) v_{2} \leq x \leq 0\right)$. It follows that if $A=\Sigma_{t} A^{\text {in }}$, then $A_{1}(t)$ and $A_{2}(t)$ are nonnegative and nondecreasing in $\mathbb{R}^{-}$for every $t \geq 0$.

As for the case $(i i)$, for $r \in \mathbb{N}^{*}$, we split $\left[X-T v_{2}, X\right] \subset \mathbb{R}^{-}$into $r$ subintervals $\left[x^{j}, x^{j+1}\right], 0 \leq j<r$, with $x^{j} \equiv X-T v_{2}(1-j / r)$ for $j \in \mathbb{Z}$ and set $t^{j} \equiv T-\left(X-x^{j}\right) / v_{2}=T / r$ for $0 \leq j \leq r$. We fix $j, k \in \mathbb{N}$ with $0 \leq j \leq k<r, k \in \mathbb{N}$ and work in the (space-time) triangle $\mathcal{D}^{j, k}$ with vertices $\left(x^{j-1}, t^{k}\right),\left(x^{j}, t^{k}\right)$ and $\left(x^{j}, t^{k+1}\right)$. We show a bound from below for $A_{1}$ and $A_{2}$ at $\left(x^{j}, t^{k}\right)$ depending on their values at $\left(x^{j-1}, t^{k}\right)$. 
First, since $A_{1}(t)$ and $A_{2}(t)$ are nonnegative and nondecreasing in $\mathbb{R}^{-}$for every $t \geq 0$, we infer as a first step that for $(t, x) \in \mathcal{D}^{j, k}$

$$
A_{1}(t, x) \geq A_{1}\left(t^{k}, x-\left(t-t^{k}\right) v_{1}\right) \geq A_{1}\left(t^{k}, x^{j-1}\right) \quad \text { and } \quad A_{2}(t, x) \geq A_{2}\left(t^{k}, x-\left(t-t^{k}\right) v_{2}\right) \geq A_{2}\left(t^{k}, x^{j-1}\right) .
$$

Consequently,

$$
\begin{aligned}
A_{1}\left(t^{k+1}, x^{j}\right) & \geq A_{1}\left(t^{k}, x^{j}-\left(t^{k+1}-t^{k}\right) v_{1}\right)-\sigma_{1} \int_{t^{k}}^{t^{k+1}} A_{2}\left(\tau, x^{j}-\left(t^{k+1}-\tau\right) v_{1}\right) A_{3}\left(x^{j}-\left(t^{k+1}-\tau\right) v_{1}\right) d \tau \\
& \geq A_{1}\left(t^{k}, x^{j-1}\right)-\frac{\sigma_{1}}{v_{1}} A_{2}\left(t^{k}, x^{j-1}\right) \int_{x^{j}-\left(t^{k+1}-t^{k}\right) v_{1}}^{x^{j}} A_{3}(y) d y
\end{aligned}
$$

(since $0 \leq v_{1} \leq v_{2}$, we have $\left.\left(\tau, x^{j}-\left(t^{k+1}-\tau\right) v_{1}\right) \in \mathcal{D}^{j, k}\right)$, and similarly

$$
\begin{aligned}
A_{2}\left(t^{k+1}, x^{j}\right) & \geq A_{2}\left(t^{k}, x^{j}-\left(t^{k+1}-t^{k}\right) v_{2}\right)-\sigma_{2} \int_{t^{k}}^{t^{k+1}} A_{1}\left(\tau, x^{j}-\left(t^{k+1}-\tau\right) v_{2}\right) A_{3}\left(x^{j}-\left(t^{k+1}-\tau\right) v_{2}\right) d \tau \\
& \geq A_{2}\left(t^{k}, x^{j-1}\right)-\frac{\sigma_{2}}{v_{2}} A_{1}\left(t^{k}, x^{j-1}\right) \int_{x^{j}-\left(t^{k+1}-t^{k}\right) v_{2}}^{x^{j}} A_{3}(y) d y .
\end{aligned}
$$

From the fact that $0 \leq v_{1} \leq v_{2}$, we deduce that the quantity

$$
\mathfrak{a}^{j, k} \equiv \min \left\{A_{1}\left(t^{k}, x^{j}\right) ; \sqrt{\frac{\sigma_{1} v_{2}}{\sigma_{2} v_{1}}} A_{2}\left(t^{k}, x^{j}\right)\right\}
$$

verifies

$$
\mathfrak{a}^{j, k+1} \geq \mathfrak{a}^{j-1, k}\left(1+\sqrt{\frac{\sigma_{1} \sigma_{2}}{v_{1} v_{2}}} \int_{x^{j}-\left(t^{k+1}-t^{k}\right) v_{1}}^{x^{j}}\left|A_{3}\right|(y) d y\right)
$$

Therefore,

$$
\begin{aligned}
\mathfrak{a}^{r, r} & \geq \mathfrak{a}^{0,0} \prod_{j=1}^{r}\left(1+\sqrt{\frac{\sigma_{1} \sigma_{2}}{v_{1} v_{2}}} \int_{x^{j}-T v_{1} / r}^{x^{j}}\left|A_{3}\right|(y) d y\right) \\
& \geq \exp \left(\sum_{j=1}^{r} \sqrt{\frac{\sigma_{1} \sigma_{2}}{v_{1} v_{2}}} \int_{x^{j}-T v_{1} / r}^{x^{j}}\left|A_{3}\right|(y) d y+\frac{C(X, T)}{r}\right) .
\end{aligned}
$$

since $\mathfrak{a}^{0,0}=1$ and $\int_{x^{j}-T v_{1} / r}^{x^{j}}\left|A_{3}\right|(y) d y \leq C_{0}(X, T) / r$. Now, since $\left|A_{3}\right|$ is nondecreasing in $\mathbb{R}^{-}$

$$
\sum_{j=1}^{r} \int_{x^{j}-T v_{1} / r}^{x^{j}}\left|A_{3}\right|(y) d y \geq \frac{T v_{1}}{r} \sum_{j=1}^{r}\left|A_{3}\right|\left(x^{j}\right) \rightarrow \frac{v_{1}}{v_{2}} \int_{X-T v_{2}}^{X}\left|A_{3}\right|(y) d y
$$

as $r \rightarrow+\infty$. Hence, passing to the limit as $r \rightarrow+\infty$ in the previous inequality yields

$$
\min \left\{A_{1}(T, X) ; \sqrt{\frac{\sigma_{1} v_{2}}{\sigma_{2} v_{1}}} A_{2}(T, X)\right\} \geq \exp \left(\frac{v_{1}}{v_{2}} \sqrt{\frac{\sigma_{1} \sigma_{2}}{v_{1} v_{2}}} \int_{X-T v_{2}}^{X}\left|A_{3}\right|(y) d y\right)
$$

This establishes (50) for some constant $c>0$ depending only on $\sigma_{1}, \sigma_{2}, v_{1}$ and $v_{2}$.

\subsection{Proof of Proposition 10}

Here, we recall that $v_{1}=0 \neq v_{2}$. Let us first recall that the telegraph equation

$$
\partial_{X Y}^{2} U+K^{2} U=0
$$

for $U=U(X, Y): \mathbb{R}^{2} \rightarrow \mathbb{R}$ and $K>0$ is a constant, has a well-known particular explicit solution given by

$$
U(X, Y) \equiv J_{0}(2 K \sqrt{X Y})
$$


where, $J_{0}$ is the Bessel function defined by

$$
J_{0}(z)=\frac{1}{\pi} \int_{0}^{\pi} \cos (z \sin (u)) d u=\sum_{j=0}^{+\infty} \frac{(-1)^{j} z^{2 j}}{(j !)^{2}} .
$$

Indeed,

$$
\partial_{X Y}^{2} U=-K^{2}\left(J_{0}^{\prime \prime}(2 K \sqrt{X Y})+\frac{J_{0}^{\prime}(2 K \sqrt{X Y})}{2 K \sqrt{X Y}}\right) \quad \text { and } \quad J_{0}^{\prime \prime}(z)+\frac{J_{0}^{\prime}(z)}{z}+J_{0}(z)=0 .
$$

Similarly, the above computation yields that

$$
A_{2}(t, x) \equiv J_{0}\left(2\left|\mathcal{A}_{3}\right| \sqrt{\frac{\sigma_{1} \sigma_{2}}{v_{2}}} \sqrt{x\left(\frac{x}{v_{2}}-t\right)}\right)
$$

solves, in $\mathbb{R}_{+} \times(0, \ell)$,

$$
\partial_{t}\left(\partial_{t}+v_{2} \partial_{x}\right) A_{2}=\sigma_{1} \sigma_{2}\left|\mathcal{A}_{3}\right|^{2} A_{2}
$$

One could then define $A_{1}$ such that $\left(A_{1}, A_{2}\right)$ solves $(5)$ in $\mathbb{R}_{+} \times(0, \ell)$, with appropriate boundary and initial data. Moreover, the Laplace method yields the asymptotic behavior of $J_{0}$ on the imaginary axis:

$$
J_{0}(i \xi) \sim \frac{\mathrm{e}^{\xi}}{\sqrt{2 \pi \xi}} \quad \text { for } \xi \rightarrow+\infty \quad(\xi \in \mathbb{R})
$$

Hence, for fixed $x \in(0, \ell)$,

$$
A_{2}(t, x) \sim \frac{c_{0}}{\left|\mathcal{A}_{3}\right| \sqrt{t}} \exp \left(2 \sqrt{\frac{\sigma_{1} \sigma_{2}}{v_{2}}}\left|\mathcal{A}_{3}\right| \sqrt{x t}\right),
$$

where the positive constant $c_{0}$ depends only on $\sigma_{1}, \sigma_{2}$ and $v_{2}$. Due to the fact that the boundary datum involved in $A_{1}$ is unbounded, the growth rate in the exponential has an extra factor 2.

\subsubsection{Proof of case (i)}

Without loss of generality, we may assume that the interval $I$ is $(0, \ell)$. Then, $(10)$ becomes

$$
\left\{\begin{array}{l}
A_{1}(t, x)=A_{1}^{\mathrm{in}}(x)-\sigma_{1} A_{3}(x) \int_{0}^{t} A_{2}(\tau, x) d \tau \\
A_{2}(t, x)=A_{2}^{\mathrm{in}}\left(x-t v_{2}\right)-\sigma_{2} \int_{0}^{t} A_{1}\left(\tau, x-(t-\tau) v_{2}\right) \bar{A}_{3}\left(x-(t-\tau) v_{2}\right) d \tau .
\end{array}\right.
$$

Choosing $A_{1}^{\text {in }} \equiv 0$ and $A_{2}^{\text {in }} \equiv 1$, we infer

$$
A_{2}(t, x)=1+\frac{\sigma_{1} \sigma_{2}}{v_{2}} \int_{x-t v_{2}}^{x}\left|A_{3}\right|^{2}(y) \int_{0}^{t-(x-y) / v_{2}} A_{2}(\theta, y) d \theta d y .
$$

It is then clear that $A_{1}$ and $A_{2}$ remain non negative for $t>0$. Moreover, for $0 \leq x \leq \ell$ and $t \geq x / v_{2}$,

$$
A_{2}(t, x) \geq 1+\frac{\sigma_{1} \sigma_{2}}{v_{2}}\left|\mathcal{A}_{3}\right|^{2} \int_{0}^{x} \int_{y / v_{2}}^{t-(x-y) / v_{2}} A_{2}(\theta, y) d \theta d y
$$

This implies, as a first step, for $0 \leq x \leq \ell$ and $t \geq x / v_{2}$,

$$
A_{2}(t, x) \geq 1
$$

Inserting this lower bound into (52) yields as a second step

$$
A_{2}(t, x) \geq 1+\frac{\sigma_{1} \sigma_{2}}{v_{2}}\left|\mathcal{A}_{3}\right|^{2} \int_{0}^{x} \int_{y / v_{2}}^{t-(x-y) / v_{2}} 1 d \theta d y=1+\frac{\sigma_{1} \sigma_{2}}{v_{2}}\left|\mathcal{A}_{3}\right|^{2} x\left(t-\frac{x}{v_{2}}\right) .
$$


Arguing by induction and using the fact that, for $n \in \mathbb{N}$,

$$
\int_{0}^{x} \int_{y / v_{2}}^{t-(x-y) / v_{2}} y^{n}\left(\theta-\frac{y}{v_{2}}\right)^{n} d \theta d y=\frac{1}{(n+1)^{2}} x^{n+1}\left(t-\frac{x}{v_{2}}\right)^{n+1},
$$

it follows that, for any $n \in \mathbb{N}$,

$$
A_{2}(t, x) \geq \sum_{j=0}^{n} \frac{1}{(j !)^{2}}\left[\frac{\sigma_{1} \sigma_{2}}{v_{2}}\left|\mathcal{A}_{3}\right|^{2}\right]^{j} x^{j}\left(t-\frac{x}{v_{2}}\right)^{j} .
$$

Letting $n \rightarrow+\infty$ and in view of the power series expansion of $J_{0}$, it comes

$$
A_{2}(t, x) \geq J_{0}\left(i \sqrt{\frac{\sigma_{1} \sigma_{2}}{v_{2}}}\left|\mathcal{A}_{3}\right| \sqrt{x\left(t-\frac{x}{v_{2}}\right)}\right) .
$$

Hence, it follows from the asymptotic behavior (51) of $J_{0}$ on the imaginary axis that for any $0<\varepsilon<\ell$, there exists $c_{\varepsilon}>0$ such that

$$
A_{2}(t, x=\ell) \geq c_{\varepsilon} \exp \left(\sqrt{\frac{\sigma_{1} \sigma_{2}}{v_{2}}}\left|\mathcal{A}_{3}\right| \sqrt{(\ell-\varepsilon) t}\right)
$$

provided $t$ is sufficiently large.

\subsubsection{Proof of case (ii)}

We consider $A_{3} \in L^{\infty}(\mathbb{R})$ and compactly supported, say in $(0, \ell)$ without loosing generality. In the regions $\{x<0\}$ and $\{x>\ell\}, A_{3}$ vanishes and $A_{2}$ is explicitable:

$$
A_{2}(t, x)=A_{2}^{\text {in }}\left(x-t v_{2}\right) \quad \text { or } \quad A_{2}(t, x)=A_{2}\left(t-\frac{x-\ell}{v_{2}}, \ell\right)
$$

if $\left(x \leq 0\right.$ or $\left.x \geq \ell+t v_{2}\right)$ or $\ell \leq x \leq \ell+t v_{2}$, thus it suffices to prove the estimate in $[0, \ell]$. As in the previous case, (10) yields

$$
A_{2}(t, x)=A_{2}^{\mathrm{in}}\left(x-t v_{2}\right)-\frac{\sigma_{2}}{v_{2}} \int_{x-t v_{2}}^{x} A_{1}^{\mathrm{in}}(y) \bar{A}_{3}(y) d y+\frac{\sigma_{1} \sigma_{2}}{v_{2}} \int_{x-t v_{2}}^{x}\left|A_{3}\right|^{2}(y) \int_{0}^{t-(x-y) / v_{2}} A_{2}(\theta, y) d \theta d y,
$$

thus, for $0 \leq x \leq \ell$,

$$
\left|A_{2}\right|(t, x) \leq\left\|A_{2}^{\mathrm{in}}\right\|_{L^{\infty}}+\frac{\left|\sigma_{2}\right|}{v_{2}} \ell\left\|A_{3}\right\|_{L^{\infty}}\left\|A_{1}^{\mathrm{in}}\right\|_{L^{\infty}}+\frac{\sigma_{1} \sigma_{2}}{v_{2}}\left\|A_{3}\right\|_{L^{\infty}}^{2} \int_{0}^{x} \int_{0}^{t}\left|A_{2}\right|(\theta, y) d \theta d y .
$$

The idea is to construct a super solution to this inequality, using a power series expansion as in the previous case. Let

$$
\Phi(t, x) \equiv \sum_{j=0}^{+\infty}\left[\frac{\sigma_{1} \sigma_{2}}{v_{2}}\left\|A_{3}\right\|_{L^{\infty}}^{2}\right]^{j} \frac{x^{j} t^{j}}{(j !)^{2}}=J_{0}\left(i \sqrt{\frac{\sigma_{1} \sigma_{2}}{v_{2}}}\left\|A_{3}\right\|_{L^{\infty}} \sqrt{x t}\right)>0 .
$$

Then, an immediate computation yields

$$
1+\frac{\sigma_{1} \sigma_{2}}{v_{2}}\left\|A_{3}\right\|_{L^{\infty}}^{2} \int_{0}^{x} \int_{0}^{t} \Phi(\theta, y) d \theta d y=\Phi(t, x)
$$

Therefore, for some constant $K_{0}$ depending only on $\sigma_{1}, \sigma_{2}, v_{2}$ and $\ell\left\|A_{3}\right\|_{L^{\infty}}$, we have, for $0 \leq x \leq \ell$,

$$
\begin{aligned}
\left\{\left|A_{2}\right|-K_{0}\left\|A^{\mathrm{in}}\right\|_{L^{\infty}} \Phi\right\}_{+}(t, x) & \leq \frac{\sigma_{1} \sigma_{2}}{v_{2}}\left\|A_{3}\right\|_{L^{\infty}}^{2} \int_{0}^{x} \int_{0}^{t}\left\{\left|A_{2}\right|-K_{0}\left\|A^{\mathrm{in}}\right\|_{L^{\infty}} \Phi\right\}(\theta, y) d \theta d y \\
& \leq \frac{\sigma_{1} \sigma_{2}}{v_{2}}\left\|A_{3}\right\|_{L^{\infty}}^{2} \ell \int_{0}^{t}\left\|\left\{\left|A_{2}\right|-K_{0}\left\|A^{\mathrm{in}}\right\|_{L^{\infty}} \Phi\right\}_{+}(\theta)\right\|_{L^{\infty}(0, \ell)} d \theta .
\end{aligned}
$$

Hence, by Gronwall inequality, for any $t \geq 0$ and $x \in[0, \ell]$,

$$
\left\{\left|A_{2}\right|-K_{0}\left\|A^{\mathrm{in}}\right\|_{L^{\infty}} \Phi\right\}(t, x) \leq 0
$$


Consequently, from the asymptotics (51),

$$
\left|A_{2}\right| \leq K_{0}\left\|A^{\mathrm{in}}\right\|_{L^{\infty}} \Phi(t, x) \leq \frac{C}{\sqrt{t}}\left\|A^{\mathrm{in}}\right\|_{L^{\infty}} \exp \left(\sqrt{\frac{\sigma_{1} \sigma_{2}}{v_{2}}}\left\|A_{3}\right\|_{L^{\infty}} \sqrt{\ell t}\right)
$$

for some constant $C$ depending on $\sigma_{1}, \sigma_{2}, v_{2}, \ell$ and $\left\|A_{3}\right\|_{L^{\infty}}$. Inserting this upper bound in the first line of (10) yields

$$
\begin{aligned}
\left|A_{1}\right|(t, x) & \leq\left\|A_{1}^{\mathrm{in}}\right\|_{L^{\infty}}+\left|\sigma_{1}\right|\left\|A_{3}\right\|_{L^{\infty}}\left\|A^{\mathrm{in}}\right\|_{L^{\infty}} \int_{0}^{t} \frac{C}{\sqrt{\tau}} \exp \left(\sqrt{\frac{\sigma_{1} \sigma_{2}}{v_{2}}}\left\|A_{3}\right\|_{L^{\infty}} \sqrt{\ell \tau}\right) d \tau \\
& \leq C^{\prime}\left\|A^{\mathrm{in}}\right\|_{L^{\infty}} \exp \left(\sqrt{\frac{\sigma_{1} \sigma_{2}}{v_{2}}}\left\|A_{3}\right\|_{L^{\infty}} \sqrt{\ell t}\right) .
\end{aligned}
$$

The proof is complete.

\subsection{Proof of Proposition 11}

We denote $\left(\tilde{x}_{1}, \tilde{x}_{2}\right)$ the coordinates in the basis $\left(v_{1} ; v_{2}\right)$ of $\mathbb{R}^{2}$. We follow closely the arguments used in the proof of Proposition 9. We fix $(T, X) \in \mathbb{R}_{+} \times \mathbb{R}^{2}$. For $r \in \mathbb{N}^{*}$ and $j=\left(j_{1}, j_{2}\right) \in \mathbb{Z}^{2}$, we denote

$$
V^{j} \equiv \tilde{X}_{1}-T\left(1-j_{1} / r\right) v_{1}+\tilde{X}_{2}-T\left(1-j_{1} / r\right) v_{2} \in \mathbb{R}^{2}
$$

We define $\mathcal{R}$ as the parallelogram with vertices $0, \tilde{X}_{2} v_{2}=V^{(0, r)}, \tilde{X}_{1} v_{1}=V^{(r, 0)}$ and $X=V^{(r, r)}$, and $\mathcal{R}^{j}\left(j \in \mathbb{Z}^{2}\right)$ as the parallelogram with vertices $V^{\left(j_{1}, j_{2}\right)}, V^{\left(j_{1}+1, j_{2}\right)}, V^{\left(j_{1}, j_{2}+1\right)}$ and $V^{\left(j_{1}+1, j_{2}+1\right)}$. We then decompose the parallelogram $\mathcal{R}$ into the $r^{2}$ subparallelograms $\mathcal{R}^{j}, j \in \mathbb{Z}^{2}, 0 \leq j_{1}, j_{2}<r$.

On each parallelogram $\mathcal{R}^{j}, A$ solves, in the space-time domain

$$
\mathcal{D}^{j} \equiv\left\{(t, x) \in \mathbb{R}^{+} \times \mathcal{R}^{j}, \tilde{x}_{1}+\tilde{x}_{2}-t \geq \tilde{X}_{1}+\tilde{X}_{2}-T\right\},
$$

the boundary value problem

$$
\left\{\begin{array}{l}
\partial_{t} A_{1}+\partial_{\tilde{x}_{1}} A_{1}+\sigma_{1} A_{3} A_{2}=0 \\
\partial_{t} A_{2}+\partial_{\tilde{x}_{2}} A_{2}+\sigma_{2} \bar{A}_{3} A_{1}=0,
\end{array} \quad \text { for } \quad(t, x) \in \mathcal{D}^{j}\right.
$$

with the boundary conditions on the faces $\mathcal{F}_{1}^{j} \equiv \mathcal{D}^{j} \cap\left\{\tilde{x}_{1}=V_{1}^{j}\right\}$ and $\mathcal{F}_{2}^{j} \equiv \mathcal{D}^{j} \cap\left\{\tilde{x}_{2}=V_{2}^{j}\right\}$ :

$$
\begin{aligned}
A_{1}\left(t, \tilde{x}_{1}=\tilde{V}_{1}^{j}, \tilde{x}_{2}\right) & =A_{1}\left(t, \tilde{x}_{1}=\tilde{V}_{1}^{j}, \tilde{x}_{2}\right) & \left(t, \tilde{x}_{1}=\tilde{V}_{1}^{j}, \tilde{x}_{2}\right) & \in \mathcal{F}_{1}^{j}, \\
A_{2}\left(t, \tilde{x}_{1}, \tilde{x}_{2}=\tilde{V}_{2}^{j}\right) & =A_{2}\left(t, \tilde{x}_{1}, \tilde{x}_{2}=\tilde{V}_{2}^{j}\right) & \left(t, \tilde{x}_{1}, \tilde{x}_{2}=\tilde{V}_{2}^{j}\right) & \in \mathcal{F}_{2}^{j}, \\
A(t=0, \tilde{x}) & =A^{\text {in }}(\tilde{x}), & \tilde{x} & \in \mathcal{R}^{j} .
\end{aligned}
$$

By the method of characteristics, we find for $(t, x) \in \mathcal{D}^{j}$ :

$$
\begin{aligned}
\left|A_{1}\right|(t, \tilde{x}) \leq & \mathbf{1}_{\tilde{x}_{1} \geq \tilde{x}_{1}^{j}+t}\left\{\left\|A_{1}^{\mathrm{in}}\right\|_{\mathcal{C}\left(\mathcal{R}^{j}\right)}+\left|\sigma_{1}\right| \int_{0}^{t}\left\|A_{3}\right\|_{L^{\infty}\left(\mathcal{R}^{j}\right)}\left\|A_{2}\right\|_{\mathcal{C}\left(\mathcal{D}^{j}\right)} d \tau\right\} \\
& +\mathbf{1}_{\tilde{x}_{1} \leq \tilde{V}_{1}^{j}+t}\left\{\left\|A_{1 \mid \tilde{x}_{1}=\tilde{V}_{1}^{j}}\right\|_{\mathcal{C}\left(\mathcal{F}_{1}^{j}\right)}+\left|\sigma_{1}\right| \int_{t-\frac{\tilde{x}_{1}-\tilde{x}_{1}^{j}}{\left|v_{1}\right|}}^{t}\left\|A_{3}\right\|_{L^{\infty}\left(\mathcal{R}^{j}\right)}\left\|A_{2}\right\|_{\mathcal{C}\left(\mathcal{D}^{j}\right)} d \tau\right\} \\
\leq & \max \left\{\left\|A_{1}^{\mathrm{in}}\right\|_{\mathcal{C}(\mathcal{R})} ;\left\|A_{1 \mid \tilde{x}_{1}=\tilde{V}_{1}^{j}}\right\|_{\mathcal{C}\left(\mathcal{F}_{1}^{j}\right)}\right\}+\left|\sigma_{1}\right| \frac{T}{r}\left\|A_{3}\right\|_{L^{\infty}\left(\mathcal{R}^{j}\right)}\left\|A_{2}\right\|_{\mathcal{C}\left(\mathcal{D}^{j}\right)}
\end{aligned}
$$

and similarly (here, the two quantities $A_{1}$ and $A_{2}$ play symmetric roles)

$$
\left|A_{2}\right|(t, \tilde{x}) \leq \max \left\{\left\|A_{2}^{\text {in }}\right\|_{\mathcal{C}(\mathcal{R})} ;\left\|A_{2 \mid \tilde{x}_{2}=\tilde{x}_{2}^{j}}\right\|_{\mathcal{C}\left(\mathcal{F}_{2}^{j}\right)}\right\}+\left|\sigma_{2}\right| \frac{T}{r}\left\|A_{3}\right\|_{L^{\infty}\left(\mathcal{R}^{j}\right)}\left\|A_{1}\right\|_{\mathcal{C}\left(\mathcal{D}^{j}\right)} .
$$

For $j \in \mathbb{Z}^{2}$, with $0 \leq j_{1}, j_{2}<r$, we set

$$
\alpha^{j} \equiv \frac{T}{r} \sqrt{\sigma_{1} \sigma_{2}}\left\|A_{3}\right\|_{L^{\infty}\left(\mathcal{R}^{j}\right)}, \quad\left\{\begin{array}{l}
\mathfrak{a}_{1}^{j} \equiv \max \left\{\left\|A_{1}^{\mathrm{in}}\right\|_{\mathcal{C}(\mathcal{R})} ;\left\|A_{1}\right\|_{\mathcal{C}\left(\mathcal{D}^{j}\right)}\right\} \\
\mathfrak{a}_{2}^{j} \equiv \sqrt{\frac{\sigma_{1} v_{2}}{\sigma_{2} v_{1}}} \max \left\{\left\|A_{2}^{\mathrm{in}}\right\|_{\mathcal{C}(\mathcal{R})} ;\left\|A_{2}\right\|_{\mathcal{C}\left(\mathcal{D}^{j}\right)}\right\}
\end{array}\right.
$$


Then, we have

$$
\left\{\begin{array}{l}
\mathfrak{a}_{1}^{\left(j_{1}, j_{2}\right)} \leq \mathfrak{a}_{1}^{\left(j_{1}-1, j_{2}\right)}+\alpha^{\left(j_{1}, j_{2}\right)} \mathfrak{a}_{2}^{\left(j_{1}, j_{2}\right)} \\
\mathfrak{a}_{2}^{\left(j_{1}, j_{2}\right)} \leq \mathfrak{a}_{2}^{\left(j_{1}, j_{2}-1\right)}+\alpha^{\left(j_{1}, j_{2}\right)} \mathfrak{a}_{1}^{\left(j_{1}, j_{2}\right)}
\end{array}\right.
$$

from which it follows

$$
\left\{\begin{array}{l}
\mathfrak{a}_{1}^{\left(j_{1}, j_{2}\right)} \leq \frac{\mathfrak{a}_{1}^{\left(j_{1}-1, j_{2}\right)}+\alpha^{\left(j_{1}, j_{2}\right)} \mathfrak{a}_{2}^{\left(j_{1}, j_{2}-1\right)}}{1-\left(\alpha^{\left(j_{1}, j_{2}\right)}\right)^{2}} \\
\mathfrak{a}_{2}^{\left(j_{1}, j_{2}\right)} \leq \frac{\mathfrak{a}_{1}^{\left(j_{1}, j_{2}-1\right)}+\alpha^{\left(j_{1}, j_{2}\right)} \mathfrak{a}_{2}^{\left(j_{1}-1, j_{2}\right)}}{1-\left(\alpha^{\left(j_{1}, j_{2}\right)}\right)^{2}}
\end{array}\right.
$$

as soon as $\alpha^{\left(j_{1}, j_{2}\right)}<1$, which is the case if $r \geq 1+T \sqrt{\sigma_{1} \sigma_{2}}\left\|A_{3}\right\|_{L^{\infty}\left(\mathbb{R}^{2}\right)}$. Let us set

$$
K^{0} \equiv \max \left\{\mathfrak{a}_{\ell}^{(i, 0)} ; \ell=1,20 \leq i \leq r\right\}
$$

By an immediate induction, we infer

$$
\mathfrak{a}_{1}^{(i, 1)} \leq K^{0} \delta_{i}
$$

where the $\delta_{i}$ 's are defined by $\delta_{0} \equiv 1$ and

$$
\delta_{i} \equiv \frac{1+\delta_{i-1} \alpha^{(i, 0)}}{1-\left(\alpha^{(i, 0)}\right)^{2}} .
$$

It can be easily checked by induction on $i$ that

$$
\delta_{i}=\left\{\prod_{h=0}^{i}\left(1-\left(\alpha^{(h, 0)}\right)^{2}\right)\right\}^{-1}\left[\left(1-\left(\alpha^{(0,0)}\right)^{2}\right) \prod_{h=0}^{i} \alpha^{(h, 0)}+\sum_{\ell=0}^{i-1} \prod_{h=\ell+2}^{i} \alpha^{(h, 0)} \times \prod_{h=0}^{\ell}\left(1-\left(\alpha^{(h, 0)}\right)^{2}\right)\right] .
$$

Therefore, using that

$$
\prod_{h=0}^{\ell}\left(1-\left(\alpha^{(h, 0)}\right)^{2}\right) \leq 1
$$

and noticing that if $C_{0} \equiv T \sqrt{\sigma_{1} \sigma_{2}}\left\|A_{3}\right\|_{L^{\infty}\left(\mathbb{R}^{2}\right)}$, then, for $0 \leq j_{1}, j_{2}<r$,

$$
\alpha^{\left(j_{1}, j_{2}\right)} \leq \frac{C_{0}}{r}
$$

we infer, for $0 \leq i<r$

$$
\begin{aligned}
\delta_{i} & \leq \exp \left(-\sum_{h=0}^{i} \ln \left(1-\left(\alpha^{(h, 0)}\right)^{2}\right)\right)\left[1+\alpha^{(i, 0)}+\frac{C_{0}^{2}}{r^{2}}+\sum_{\ell=0}^{i-3}\left(\frac{C_{0}^{2}}{r^{2}}\right)^{i-\ell-1}\right] \\
& \leq \exp \left(\alpha^{(i, 0)}-\sum_{h=0}^{i} \ln \left(1-\left(\alpha^{(h, 0)}\right)^{2}\right)+\frac{C_{0}^{\prime}}{r^{2}}\right) .
\end{aligned}
$$

Reporting this into (57) yields

$$
\mathfrak{a}_{1}^{(i, 1)} \leq K^{0} \exp \left(\max _{0 \leq h \leq r} \alpha^{(h, 0)}-\sum_{h=0}^{r} \ln \left(1-\left(\alpha^{(h, 0)}\right)^{2}\right)+\frac{C_{0}^{\prime}}{r^{2}}\right)
$$

and, by similar arguments,

$$
\mathfrak{a}_{1}^{(1, i)} \leq K^{0} \exp \left(\max _{0 \leq h \leq r} \alpha^{(0, h)}-\sum_{h=0}^{r} \ln \left(1-\left(\alpha^{(0, h)}\right)^{2}\right)+\frac{C_{0}^{\prime}}{r^{2}}\right) .
$$

Consequently, denoting $\hat{\alpha}^{\left(j_{1}, j_{2}\right)} \equiv \max \left\{\alpha^{\left(j_{1}, j_{2}\right)} ; \alpha^{\left(j_{2}, j_{1}\right)}\right\}$, it comes

$$
\max \left\{\mathfrak{a}_{\ell}^{(i, 1)} ; \ell=1,21 \leq i \leq r\right\} \leq K^{1} \equiv K^{0} \exp \left(\max _{0 \leq h \leq r} \hat{\alpha}^{(h, 0)}-\sum_{h=0}^{r} \ln \left(1-\left(\hat{\alpha}^{(h, 0)}\right)^{2}\right)+\frac{C_{0}^{\prime}}{r^{2}}\right)
$$


which is an estimate analoguous to (56). Hence, arguing by induction on the lines $j_{1}=C t e$ and $j_{2}=C t e$, we deduce

$$
\max \left\{\mathfrak{a}_{\ell}^{\left(j_{1}, j_{2}\right)} ; \ell=1,21 \leq j_{1}, j_{2} \leq r\right\} \leq K^{0} \exp \left(\sum_{i=0}^{r} \max _{i \leq h \leq r} \hat{\alpha}^{(h, i)}-\sum_{0 \leq i \leq h \leq r} \ln \left(1-\left(\hat{\alpha}^{(h, i)}\right)^{2}\right)+\frac{C_{0}^{\prime}}{r}\right) .
$$

We now let $r \rightarrow+\infty$ in the previous estimate $\left(C_{0}^{\prime}\right.$ depends on $T$ ). We bound $K^{0}$ using that (for $\ell=1,2$ )

$$
\mathfrak{a}_{\ell}^{(i, 0)} \rightarrow\left\|A_{\ell}^{\mathrm{in}}\right\|_{L^{\infty}(\mathcal{R})}
$$

uniformly for $0 \leq i \leq r$ (since in the $L^{\infty}$ norm, the time interval shrinks to $\{0\}$ ). Moreover, in view of (14), there holds

$$
\sum_{i=0}^{r} \max _{i \leq h \leq r} \hat{\alpha}^{(h, i)}-\sum_{0 \leq i \leq h \leq r} \ln \left(1-\left(\hat{\alpha}^{(h, i)}\right)^{2}\right) \leq C(\nu, M),
$$

where $C(\nu, M)$ depends on $\nu$ and $M$ (and $v_{1}, v_{2}$ ), but not on $X, T$ or $r$. Therefore, for some constant $C$ depending only on $\sigma_{1}$ and $\sigma_{2}$, we have as wished

$$
\frac{1}{C}|A(T, X)|_{\infty} \leq \max \left\{\mathfrak{a}_{1}^{(r-1, r-1)} ; \mathfrak{a}_{2}^{(r-1, r-1)}\right\} \leq\left\|A^{\text {in }}\right\|_{L^{\infty}(\mathcal{R})} \mathrm{e}^{C(\nu, M)}
$$

\section{References}

[1] M. Ablowitz, D. Kaup, A. Newell and H. Segur, The inverse scattering transform-Fourier analysis for nonlinear problems. Studies in Appl. Math. 53, no. 4 (1974), 249-315.

[2] F. Baronio, M. Conforti, M. Andreana, V. Couderc, C. De Angelis, S. Wabnitz, A. Barthélémy And A. Degasperis, Frequency Generation and Solitonic Decay in Three-Wave Interactions. Opt. Express 17 (2009), 13889-13894.

[3] R. Beals and R. Coifman, Inverse scattering and evolution equations. Comm. Pure Appl. Math. 38, no. 1 (1985), 29-42.

[4] D. Chiron, On the Schrödinger approximation for wavetrains in semilinear equations. In preparation.

[5] E. Coddington and N. Levinson, Theory of ordinary differential equations. McGraw-Hill Book Company, Inc., New York-Toronto-London, (1955).

[6] A. Craik, Wave interactions and fluid flows. Cambridge Monographs on Mechanics and Applied Mathematics. Cambridge University Press, Cambridge, (1985).

[7] D. Kaup, The inverse scattering solution for the full three-dimensional three-wave resonant interaction. Phys. D 1, no. 1 (1980), 45-67.

[8] D. Kaup, A. Rieman And A. Bers, Space-time evolution of nonlinear three-wave interactions. I. Interaction in a homogeneous medium. Rev. Mod. Phys. 51, no. 2 (1979), 275-309.

[9] G. SchneIder, Justification and failure of the nonlinear Schrödinger equation in case of non-trivial quadratic resonances. J. Differential Equations 216, no. 2 (2005), 354-386.

[10] H. Triebel, Interpolation theory, function spaces, differential operators. Johann Ambrosius Barth, Heidelberg, (1995) (second edition).

[11] K. Yosida, Lectures on differential and integral equations. Pure and Applied Mathematics, Vol. X Interscience Publishers, New York-London (1960).

[12] V. ZaKharov, Exact solutions to the problem of the parametric interaction of three-dimensional wave packets. Dokl. Akad. Nauk. S.S.S.R. 228 (1976), 1314-1316. [Translated in Soviet Phys. Dokl. 21 (1976), 322-323].

[13] V. Zakharov and S. Manakov, Resonant interaction of wave packets in nonlinear media. Zh. Eksp. Tero. Fiz. Pis'ma Red. 18, (1973), 413-417 . [Translated in JETP Lett. 18, no. 7 (1973), 243-245]. 
[14] V. Zakharov And S. Manakov, The theory of resonance interaction of wave packets in nonlinear media. Zh. Eksper. Teoret. Fiz. 69, no. 5 (1975), 1654-1673. [Translated in Soviet Physics JETP 42, no. 5 (1975), 842-850].

[15] X. ZHOU, Direct and inverse scattering transforms with arbitrary spectral singularties. Comm. Pure Appl. Math. 42 (1989), 895-938.

[16] X. ZHou, $L^{2}$-Sobolev space bijectivity of the scattering and inverse scattering transforms. Comm. Pure Appl. Math. 51 (1998), 697-731. 